\title{
2D Frozen spin method of searching for the deuteron EDM in a storage ring
}

by

Alexander Aksentev

A Dissertation

Submitted to the department of electrophysical facilities

National Research Nuclear University "MEPhI"

in partial fulfillment of the requirements

for the degree of

Physics - Doctor of Philosophy 


\section{Contents}

$\begin{array}{ll}\text { Contents } & 2\end{array}$

Introduction 4

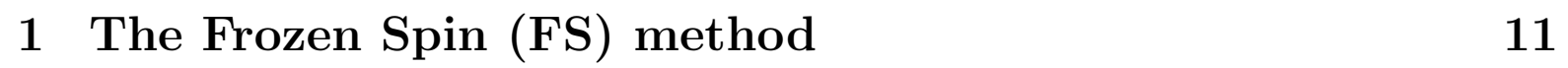

1.1 General introduction . . . . . . . . . . . . . . . . 11

1.2 FS-based methodologies . . . . . . . . . . . . . . . . . 13

1.3 Frozen- and Quasi-frozen spin lattice . . . . . . . . . 26

2 Universal SR EDM measurement problems and their solutions 31

2.1 Perturbations to the spin dynamics . . . . . . . . . . . 31

2.2 Spin decoherence . . . . . . . . . . . . . . . . . . . 36

2.3 Machine imperfections error . . . . . . . . . . . . . . . . 45

$2.4 \quad$ Guide field flipping . . . . . . . . . . . . . . . . . . . . 48

$2.5 \quad$ Spin tune equivalence of trajectories of equal effective Lorentz

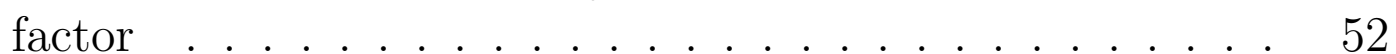

$\begin{array}{llr}3 & \text { Results at COSY } & 83\end{array}$

$3.1 \quad$ Synchrotron COSY . . . . . . . . . . . . . . . . . . . 83

3.2 High precision spin tune measurement . . . . . . . . . . 85

$3.3 \quad$ Beam Based Alignment . . . . . . . . . . . . . . . . . . 85

3.4 Spin coherence time optimization procedure $\ldots \ldots . . \quad 87$

\begin{tabular}{ll}
\hline Conclusion & 95
\end{tabular} 
\begin{tabular}{ll}
\hline Appendix A Statistical modeling & 97
\end{tabular}

A.1 Preliminary analysis . . . . . . . . . . . . . . . . . . 98

A.2 Detector counting rate model . . . . . . . . . . . . . . . 102

A.3 Cross section asymmetry . . . . . . . . . . . . . . . . . . . 104

A.4 Measurement time frame . . . . . . . . . . . . . . . . . . 104

A.5 Simulation . . . . . . . . . . . . . . . . . . . 107

\begin{tabular}{ll}
\hline Bibliography & 111
\end{tabular} 


\section{Introduction}

One of the main problems of contemporary fundamental physics research is the baryon asymmetry of the universe, i.e. the prevalence of matter over antimatter in the observed universe. At the present moment there's no evidence of the existence of primordial antimatter in our galaxy; the amount of observed antimatter is consistent with its production in secondary processes. There's also no detectable background gamma radiation that would be expected from nucleon-antinucleon annihilations, if matter and antimatter galaxies were to coexist in clusters of galaxies. [1]

In his 1967 paper, Andrei D. Sakharov formulated three necessary conditions that the primordial universe must have satisfied for baryogenesis. The discovery of cosmic background radiation and CP-symmetry violation in kaon systems [2] motivated the formulation of the conditions. The three Sakharov conditions are:

- violation of the baryon number symmetry;

- violation of the discrete $\mathrm{C}$ - and $\mathrm{CP}$-symmetries;

- a departure from thermal equilibrium.

Permanent electric dipole moments (EDMs), if they exist, violate both the $\mathrm{P}$ - and T-symmerties, and hence, via the CPT-theorem, can be linked to CP-symmetry violation.

The Standard Model (SM) of elementary particles allows formalization of CP-invariance viloation via the Cabibbo-Kobayashi-Masakawa matrix, however the EDM values it predicts for, say, the neuteron, lie in the range $10^{-33}$ to $10^{-30} e \cdot \mathrm{cm}$. [3] This implies that particle EDMs can serve as a 
powerful tool for discovering physics beyond the SM. For example, CPviolations that are endemic in supersymmetric teories (SUSY) are such as to span $d_{n}$ values in the range of $10^{-29}$ to $10^{-24} e \cdot \mathrm{cm}$. [4]

The EDM search project started more than 50 years ago. The first neuteron EDM experiment was conducted by dr. N.F. Ramsey at the end of the 1950s. As a result of that experiment, the upper neuteron EDM bound was set at $5 \cdot 10^{-20} e \cdot \mathrm{cm}$. [5] Since then, multiple experiments of increasingly high precision were carried out, and at present the upper bound on the neuteron EDM is at $2.9 \cdot 10^{-26} e \cdot \mathrm{cm}$. [6, 7]

Up until now, all EDM-searching experiments were performed on electricallyneutral particles, such as atoms or the neuteron. The idea of searching of a charged particle's EDM in a storage ring environment appeared during the development of the g-2 experiment [8] in Brookhaven National Laboratory.

As a result of the BNL experiments, the upper bound on the muon EDM was set at $10^{-19} e \cdot \mathrm{cm}$. [9] In the 1990s, discussion centered mostly on the muon EDM experiment [10], however the deuteron was also considered, in view of its having a similar magnetic anomaly-to-mass ratio.

In 2004, the Storage Ring EDM Collaboration (srEDM) [11] in BNL proposed experiment 970 for detecting the deuteron EDM on the level $10^{-27} e \cdot c \mathrm{cmin}$ a storage ring. Since 2005 , a number of feasibility experiments were run at the KVI cyclotron facility in Groningen to measure broad range spin sensitivities for deuteron scattering on carbon near $100 \mathrm{MeV}$.

Experiments at the Cooler Synchrotron COSY (Forschungszentrum Jülich, Germany) began in 2008. Later these experiments developed into a polarized program at COSY with a view of delveloping technologies required for a storage ring EDM search experiment. In the same year another deuteron EDM experiment was proposed [12], this time with a sensitivity level $10^{-29} e \cdot \mathrm{cmin}$ one year of measurement time.

At the same time it was decided that the proton EDM experiment has a number of technical advantages ober the deuteron. Among them is the ability to store two counter-circulating beams simultaneously, which allows the cancellation of T-even systematic effects. Nevertheless, work at COSY continued with the deuteron because of the investment already made in deuteron operation and the sense that any conclusions would apply to 
either proton or deuteron beams. [13, Historical background]

In 2011 the JEDI (Jülich Elecric Dipoe moment Investigations) collaboration was formed. [4] The purpose of the collaboration consists not only in developing technologies for srEDM, but also in performing a first direct EDM measurement for deuterons.

In 2018, the JEDI collaboration made a first-ever deuteron EDM measurement at COSY. Since in a non-Frozen Spin ring and EDM generates small-amplitude oscillations of the vertical beam polarization component (at the deuteron momentum $970 \mathrm{MeV} / \mathrm{c}$ used in COSY, the oscillation amplitude is on the level $3 \cdot 10^{-10}$ assuming an EDM $d=10^{-24} e \cdot \mathrm{cm}$ ), a resonance-type methodology was used [14, 15], which uses a custom-design RF Wien filter [16, 17] made specially for COSY.

The goal of the present work is to numerically model the 2D Frozen Spin (FS) method for searching for the deuteron EDM in a storage ring.

In order to reach this goal, the following objectives were formulated:

(1) Study spin decoherence in the neigborhood of spin resonance (frozen spin regime) and the sextupole method of its suppression.

(2) Study the effect of betatron oscillations on the validity of the EDM statstic.

(3) Study the effects of lattice optical element misalignments on the systematic error of the EDM statistic.

(4) Model the spin tune calibration procedure used at flipping the polarity of the storage ring guide field when counter-injecting the beam.

This research deals with the following problems not analyzed by previous researchers:

(1) Simulation of the spin tune calibration procedure under change of the beam circulation direction.

(2) Analysis of the effect of betatron oscillations on the EDM statistic. 
(3) Systematization of the universally-encountered EDM measurement problems.

(4) Classification of FS-type methods of searching for the EDM of a charged particle in a storage ring.

\section{Defended propositions.}

(1) We confirmed the analytical explanation of the mechanism of the sextupole method for suppressing spin decoherence proposed by Y.V. Senichev.

(2) We confirmed the proposed equality of spin tunes of particles having the same value of the effective Lorentz factor, and we found an interpretation fo the effective Lorentz factor as a measure of the particle's longitudinal emittance.

(3) We showed that the calibration of the vertical MDM precession angular velocity component by means of observing spin precession in the horizontal plane is a viable method.

(4) We proved that perturbations to a particle's spin dynamics due to its betatron motion introduce a negligibly small (and also controllable, in the framework of the 2D FS method) systematic error into the EDM statistic.

(5) We proved that the effective measurement cycle length ranges betwen 2 to 3 polarization lifetimes. 1

(6) We showed the possibility of reaching a mean standard error on the EDM on the level of $10^{-29} e \cdot c$ min one year of measurement.

(7) We proved that the EDM-faking MDM spin precession angular velocity due to machine imperfections is independent of the actual

\footnotetext{
${ }^{1}$ Polarization lifetime here is understood as the period of time during which polarizationi drops by a factor of $e$.
} 
distribution of the imperfections 2 , and depends only on the expectation value of the imperfection distribution.

(8) We proved that at the practical level of element alignment precision non-frequency based EDM measurement methodologies cannot be used.

Structure of the dissertation. This dissertation consists of an introduction, three chapters, a conclusion, and one appendix.

\section{Chapter one:}

(1) Introduces the Frozen Spin concept.

(2) Provides a classification of frozen spin (FS) storage ring (SR) EDM search methodologies.

(3) Classifies some problems encountered in any FS SR EDM experiment.

(4) Describes the 2D FS method which aims to provide a solution to those problems.

(5) Describes some lattices that could be used with the proposed method.

In the second chapter we alnalyze the problems outlined in the first chapter, and their solutions; simuilation results follow.

Considered problems:

(1) perturbations in the particle spin dynamics caused by betatron oscillations, and their effect on the EDM-statistic of the 2D FS method;

(2) spin decoherence in the frozen spin regime;

(3) properties ang magnitude of the EDM-faking spin precession systematic error induced by machine imperfections;

\footnotetext{
${ }^{2}$ In the particular imperfection case considered
} 
(4) the guide field flipping procedure used for the elimination of the systematic error within the 2D FS methodology.

A section is dedicated to the investigation of the question of interpretation of the notion of the effective Lorentz factor $\left(\gamma_{\text {eff }}\right)$ introduced in the first chapter.

This notion is the foundation of a great deal of the 2D FS methodology. It can be defined as follows: if two particles' $\gamma_{e f f}$ are equal, then their spin dynamics are equivalent (specifically, their spin precession angular velocity vectors have equal orientations and magnitudes), regardless of the particulars of their orbital motion.

Specifically, what enables us to exclude the EDM-faking MDM precession from the 2D FS EDM-statistic is the fixing of the $\gamma_{e f f}$ characterizing the beam.

In chapter three we highlighted some of the more important (in the context of the present research) technologies developed within the EDM search project carried out at the Cooler Synchrotron (COSY); we also described the results of the spin coherence time (SCT) optimization studies done at COSY during the April-May 2019 beamtime.

One phenomenon worth noting is the SCT change observed during prolonged (destructive) polarimetry measurements, which is (presumably) caused by the transition from the outer (halo) to the inner (core) beam layers. The observation of this phenomenon can be explained within the bounds of the sextupole spin decoherence suppression theory outlined in this dissertation.

In the conclusion, we summarize some fo the main thesis results:

(1) Effects of spin dynamics that could potentially result in systematic error were studiedm such as:

- betatron motion-related psrticle spin dynamics pertubrations;

- spin decoherence;

- machine imperfection-related EDM-faking MDM spin precession. 
(2) For each of the systematic errors, a solution was described, its effectiveness numerically analyzed.

(3) Were formulated:

- the notions of the space and time domains (with respect to the FS SR EDM measurement methodology);

- the notion of the 2D frozen spin state;

- the necessary conditions of a successful SR EDM measurement;

- the 2D FS (Frequency Domain) method, satisfying all of the necessary conditions we found.

(4) Frozen and Quasi-Frozen spin lattices were described.

The main body of the dissertation does not include our statistical analysis of the experiment; it is placed in appendix A. Two aspects of that analysis worth mentioning are: investigation of the possibility to use a non-uniform polarization sampling scheme in order to optimize the beam lifetime; determiation of the maximum effective measurement cycle duration.

As a result, we came to the conclusion that the non-uniform sampling scheme is not practical, due to the nature of polarimetry measurement. Concerning the optimal measurement cycle length, it cannot exceed three beam polarization life times. 


\section{Chapter 1}

\section{The Frozen Spin (FS) method}

\subsection{General introduction}

\section{The T-BMT equation}

The Thomas-Bargmann-Michel-Telegdi equation describes the dynamics of a spin vector $\boldsymbol{s}$ in a magnetic field $\boldsymbol{B}$ and electrostatic field $\boldsymbol{E}$. Its generalized version, which includes the EDM effect, can be written as (in the beam rest frame): [18, p. 6]

$$
\frac{\mathrm{d} \boldsymbol{s}}{\mathrm{d} t}=\boldsymbol{s} \times\left(\boldsymbol{\Omega}_{M D M}+\boldsymbol{\Omega}_{E D M}\right),
$$

where the MDM and EDM angular velocities $\boldsymbol{\Omega}_{M D M}$ and $\boldsymbol{\Omega}_{E D M}$

$$
\begin{aligned}
& \boldsymbol{\Omega}_{M D M}=\frac{q}{m}\left[G \boldsymbol{B}-\left(G-\frac{1}{\gamma^{2}-1}\right) \frac{\boldsymbol{E} \times \boldsymbol{\beta}}{c}\right], \\
& \boldsymbol{\Omega}_{E D M}=\frac{q}{m} \frac{\eta}{2}\left[\frac{\boldsymbol{E}}{c}+\boldsymbol{\beta} \times \boldsymbol{B}\right] .
\end{aligned}
$$

In the equations above, $m, q, G=(g-2) / 2$ are respectively the particle mass, charge, and anomalous magnetic moment; $\beta=v_{0} / c$, is its relative velocity factor; $\gamma$ its Lorentz factor. The EDM factor $\eta$ is defined by $d=\eta \frac{q}{2 m c} s$, where $d$ is the particle EDM, $s$ its spin. 
In the standard formalism it is usual to operate with the (rotational) one-turn spin transfer matrix: [15, p. 4]

$$
\mathbf{t}_{R}=\exp \left(-i \pi \nu_{s} \boldsymbol{\sigma} \cdot \bar{n}\right)=\cos \pi \nu_{s}-i(\boldsymbol{\sigma} \cdot \bar{n}) \sin \pi \nu_{s},
$$

where $\nu_{s}=\Omega_{s} / \Omega_{c y c}$, the ratio of the partile's spin precession frequency to its cyclotron frequency, is termed spin tune, $\bar{n}$ defines the spin precession axis, and is called the invariant spin aixs.

\section{Frozen spin concept}

From equation (1.1b) one can see that, in the absence of an EDM, the direction of a particle's spin vector can be fixed relative its momentum vector, i.e. $\boldsymbol{\Omega}_{M D M}=\mathbf{0}$; in other words, one can realize the Frozen Spin condition.

The advantage of working in the FS-regime: according to equations eqs. (1.1a) to (1.1c), the MDM and EDM angular velocity vectors are orthogonal, meaning that htey add in squares in the net frequency, and hence the frequency shift associated with the EDM becomes a second-order effect: [19, p. 5]

$$
\omega \propto \sqrt{\Omega_{M D M}^{2}+\Omega_{E D M}^{2}} \approx \Omega_{M D M}+\frac{\Omega_{E D M}^{2}}{2 \Omega_{M D M}} .
$$

This circumstance significantly diminishes the experimental sensitivity.

However, by freezing the particle's spin in the horizontal plane, the only remaining MDM angular velocity component is aligned with the EDM component, and hence adds to it linearly, which greatly improves the sensitivity.

\section{Realization of the FS condition in a storage ring}

Storage rings can be classified into three groups:

(1) Purely magnetic (COSY, NICA, etc),

(2) purely electrostatic (Brookhaven AGS Analog Ring), 
(3) combined.

In view of equation $1.1 \mathrm{~b}$, the FS condition cannot be realized in a purely magnetic ring.

For particles like the proton (whose $G>0$ ), a purely electrostatic ring can be used in the FS methodological framework, if the beam has the so-called "magic" energy, defined as $\gamma_{\text {mag }}=\sqrt{(1+G) / G}$.

For particles whose $G<0$ (deuteron) this is impossible, and one is required to use a combined ring. To realize the FS condition in a combined ring, a radial electric field is introduced [12]:

$$
E_{r}=\frac{G B_{y} c \beta \gamma^{2}}{1-G \beta^{2} \gamma^{2}}
$$

\section{$1.2 \quad$ FS-based methodologies}

In this section we first give two examples of foundational methods for searching for the EDM in a storage ring, both of which are based on the FS idea; then we generalize these methods to two mutually-exclusive categories; we finish with the introduction of the 2D FS method.

We note, too, that apart from the FS method alternative approaches to the EDM measurement exist, for example [15, 14], in which the beam polarization freely precesses about the vertical guiding field of the storage ring.

\section{BNL FS method}

The BNL FS method was proposed by the Storage Ring EDM Collaboration of Brookhaven National Laboratory in 2008. [12] It is a combined ring method. A longitudinally-polarized beam is injected into the ring; with polarimetry measurements, is spin precession is probed in the horizontal and vertical planes; The EDM signal is the change in the vertical 
polarization component over time, which is expressed by: [12, p. 8]

$$
\Delta P_{V}=P \frac{\omega_{e d m}}{\Omega} \sin \left(\Omega t+\Theta_{0}\right)
$$

where $\Omega=\sqrt{\omega_{e d m}^{2}+\omega_{a}^{2}}, \omega_{a}, \omega_{e d m}$ are the angular velocities generated by, repsectively, the magnetic and electric dipole moments.

By applyting a radial electric field $E_{r}$ (magnitude defined by equation $(1.2)$ ), it is expected that the $\omega_{a}$ component is attenuated by at least a factor of $10^{9}$; in view of the smallness of the hypothesized value $\omega_{e d m}$, $\Delta P_{V} \approx P \omega_{e d m} t$, and hence the maximum amplitude $\Delta P_{V}$ is amplified by $10^{9}$.

The expreriment is expected to reach a sensitivity level of $10^{-29} \mathrm{e} \cdot \mathrm{cm}$ in $10^{7}$ seconds (6 months) of total measurement time. At this sensitivity level cross section asymmetry $\varepsilon_{L R} \approx 5 \cdot 10^{-6}$ for the smallest practical calues of $\omega_{a}$. [12, p. 18] The latter circumstance creates a serious problem for polarimetry. [19] One way to solve it lies in applying a radial magnetic field and measuring the net MDM+EDM spin precession frequency. This is the basic idea of the so-called Spin Wheel method (also called Koop Wheel), which is considered in the next section.

The only presently known first-order systematic effect of spin dynamics is the presence of a non-zero average vertical electric field component $\left\langle E_{V}\right\rangle$. In this case, spin precesses about the radial axis at a rate [12, p. 11]

$$
\omega_{\text {syst }} \approx \frac{\mu\left\langle E_{V}\right\rangle}{\beta c \gamma^{2}} .
$$

Two circumstances are important here:

- the presence of $\left\langle E_{V}\right\rangle \neq 0$ is due to lattice element alignment error;

- This systematic effect changes sign when the beam is injected in the opposite direction.

The latter is why the clockwise/anti-clockwise beam injection pattern is used in the 2D FS method. Even though $\omega_{\text {syst }}$ changes sign when the beam circuation direction is reversed (and hence is susceptible to control), this 
methodology does not account for its magnitude. In section 2.3 (numerically, in 2.3), show that at a realistic element element alignment error standard deviation of $100 \mu \mathrm{m}$, the rate at which spin precesses about the radial axis due to hte MDM is on the level of 50-100 rad/sec. [20] Because of that, it is impossible to use this methodology in its original form.

We should also mention that attempts at reducing $\omega_{\text {syst }}$ only increase the influence of the so-called geometric phase error. [21, p. 6]

\section{Spin Wheel method}

The problems with polarimetry and high spin precession rate highlighted above are solved in the Spin Wheel method proposed by I. Koop (Novosibirsk State University). [22] The main idea behind the method consists in the following: first, the FS condition is satisfied; then, a radial magnetic field $B_{x}$ is turned on, whose magnitude is sufficient to induce spin precession at a rate of about $1 \mathrm{~Hz}$. Since the field is radial the MDM precession it causes is aligned with with the EDM one, and hence they add linearly: $\omega \propto \Omega_{M D M}+\Omega_{E D M}$.

The EDM contribution to the net precession frequency is extracted by comparing cycles with opposite sign $B_{x}$ : [22, p. 1963]

$$
\Omega_{E D M}=\frac{\Omega_{x}\left(+B_{x}\right)+\Omega_{x}\left(-B_{x}\right)}{2} .
$$

The external magnetic field also causes a vertical orbit shift. [22, p. 1963] This shift can be detected at the pico-meter level by SQUID magnetometers; it is proposed to be used for the calibration of the applied field.

Since, due to the external field, the precession about the radial axis is 10 times faster than in the original proposal,the task for polarimetry is greatly simplified. However, there have been voiced doubts regarding the possibility of measuring the field-induced orbit shift even by means of SQUIDs.

Also, the problem of machine imperfection-indeuced vertical plane precession is not solved. 


\section{General classification of FS-type methods}

Storage ring-based methods of searching for the EDMs of elementary particles can be classified into two major categories, which we will call (1) space domain, and (2) frequency domain methods.

In the space domain frmework, one measures a change in the spatial orientation of the beam polarization vector caused by the EDM.

The original storage ring, frozen spin-type method, proposed in [12], is a canonical example of a methodology in the space domain: an initially longitudinally-polarized beam is injected into the storage ring; the vertical component of its polarization vector is observed. Under ideal conditions, any tilting of the beam polarization vector from the horizontal plane is attributed to the action of the EDM.

Two technical difficulties are readily apparent with this approach:

(1) it poses a challenging task for polarimetry [19];

(2) it puts very stringent constraints on the precision of the accelerator optical element alignment.

The former is due to the requirement of detecting a change of about $5 \cdot 10^{-6}$ to the cross section asymmetry $\varepsilon_{L R}$ in order to get to the EDM sensitivity level of $10^{-29} \mathrm{e} \cdot \mathrm{cm}$. [12, p. 18]

The latter is to minimize the magnitude of the vertical plane magnetic dipole moment (MDM) precession frequency: [12, p. 11]

$$
\omega_{\text {syst }} \approx \frac{\mu\left\langle E_{v}\right\rangle}{\beta c \gamma^{2}},
$$

induced by machine imperfection fields. According to estimates done by Y. Senichev, if it is to be fulfilled, the geodetic installation precision of accelerator elements must reach $10^{-14} \mathrm{~m}$. Today's technology allows only for about $10^{-4} \mathrm{~m}$.

At the practical level of element alignment uncertainty, $\omega_{\text {syst }} \gg \omega_{\text {edm }}$, and changes in the orientation of the polarization vector are no longer EDM-driven. 
Another crucial problem one faces in the space domain is geometric phase error. [21, p. 6] The problem here lies in the fact that, even if one can somehow make field imperfections (either due to optical element misalignment or spurious electromagnetic fields) zero on average, since spin rotations are non-commutative, the polarization rotation angle due to them will not be zero.

By contrast, the frequency domain methodology 1 is founded on measuring the EDM contribution to the total (MDM and EDM together) spin precession angular velocity.

The polarization vector is made to roll about a nearly-constant, definite direction vector $\bar{n}$, with an angular velocity that is high enough for its magnitude to be easily measureable at all times. Apart from easier polarimetry, the definiteness of the angular velocity vector is a safeguard against geometric phase error.

This "Spin Wheel" may be externally applied [22], or otherwise the machine imperfection fields may be utilized for the same purpose (wheel roll rate determined by equation (1.4)). The latter is made possible by the fact that $\omega_{\text {syst }}$ changes sign when the beam revolution direction is reversed. [12, p. 11]

\section{Universal SR EDM measurement problems}

By way of introduction to the 2D FS measurement methodology, let us briefly summarize some measurement problems encountered by any EDM experiment performed in a storage ring; they can be grouped into two big categories:

- Problems solved by a Spin Wheel:

- spurious electro-magnetic fields;

- betatron motion.

- Problems having specific solutions:

\footnotetext{
${ }^{1}$ To which the $2 \mathrm{D}$ FS method belongs.
} 
- spin decoherence;

- machine imperfections.

\section{Perturbations to the spin dynamics}

Problems from the first category are ones introducing geometric phase error. Indeed, both the spurious and the focusing fields, when acting on a betatron-oscillating particle, perturb the direction and magnitude of its spin precession angular velocity vector. The effect is a spin kick in the direction defined by the perturbation.

Assume that the EDM provides a spin kick about the radial $\left(\hat{x}^{-}\right)$axis. The magnitude of the angular velocity vector has a general form

$$
\omega=\sqrt{\omega_{x}^{2}+\omega_{y}^{2}+\omega_{z}^{2}}
$$

where $\omega_{y}$ is minimized by fulfilling the frozen spin condition; $\omega_{z}$ (the constant part of which is due to machine imperfections) can be minimized via the installation of a longitudinal solenoid on the optic axis. ${ }^{2}$ In the space domain, one also tries to minimize the $\omega_{\left\langle E_{v}\right\rangle}$ contribution to $\omega_{x}=\omega_{e d m}+\omega_{\left\langle E_{v}\right\rangle}$. Consequently, spin kicks must be minimized to (significantly) less than $\omega_{e d m}$, so as to reduce geometric phase to less than the accumulated EDM phase.

The benefit of having a Spin Wheel aligned with the EDM angular velocity is that orthogonal MDM contributions to the total angular velocity

\footnotetext{
${ }^{2} 1 \mathrm{~m}$ long, magnetic field approximately $10^{-6} \mathrm{~T}$.
} 
vector add up in squares, and hence their effect is greatly diminished:

$$
\begin{aligned}
\omega & =\sqrt{\left(\omega_{e d m}+\omega_{S W}\right)^{2}+\omega_{y}^{2}+\omega_{z}^{2}} \\
& \approx\left(\omega_{e d m}+\omega_{S W}\right) \cdot\left[1+\frac{\omega_{y}^{2}+\omega_{z}^{2}}{\omega_{S W}^{2}}\right]^{1 / 2} \\
& \approx\left(\omega_{e d m}+\omega_{S W}\right) \cdot\left(1+\frac{\omega_{y}^{2}+\omega_{z}^{2}}{2 \omega_{S W}^{2}}\right) \\
& \approx \omega_{S W}+\omega_{e d m}+\underbrace{\frac{1}{2} \frac{\omega_{y}^{2}+\omega_{z}^{2}}{\omega_{S W}}}_{\epsilon} .
\end{aligned}
$$

Since our goal is to observe the EDM-related value shift in $\omega$, we need to minimize random variable $\epsilon$ :

$$
\frac{1}{2} \frac{\omega_{y}^{2}+\omega_{z}^{2}}{\omega_{S W}}<\omega_{e d m}
$$

Let's make some preliminary estimates. Suppose $\omega_{S W} \approx 50 \mathrm{rad} / \mathrm{sec}$ (the reason for choosing this value will be explained shortly), $\omega_{\text {edm }} \approx 10^{-9}$ $\mathrm{rad} / \mathrm{sec}$ (corresponding to the EDM value $10^{-29} e \cdot \mathrm{cm}$ ). Then, $\omega_{y}^{2}+\omega_{z}^{2}$ must be reduced to less than $10^{-7} \mathrm{rad} / \mathrm{sec}$, or equivalently, either angular velocity to less than $3 \cdot 10^{-4} \mathrm{rad} / \mathrm{sec}$. This is several orders of magnitude greater than the expected standard error on the angular velocity estimate, [23] and hence should not be a problem to achieve.

One case left to be considered is MDM spin kicks about the $\hat{x}$-axis. These are not attenuated, and cause the most trouble. They come in three varieties: (i) permanent, not caused by optical element misalignments; (ii) semi-permanent, caused by element tilts about the optic axis; (iii) spurious.

Semi-permanent radial spin kicks (be they caused by magnetic or electric fields) change sign when the beam revolution direction is reversed from clockwise (CW) to counter-clockwise (CCW). Spurious kicks can be dealt with by statistical averaging. Permanent, insensitive to either the guide 
field or the beam circulation direction, cannot be controlled. On the bright side, their sources should not be present under normal circumstances.

We consider the question of the influence perturbations to the spin dynamics have on the EDM-statistic in the 2D FS method in section 2.1.

\section{Spin decoherence}

Spin coherence is a measure or quality of preservation of polarization in an initially fully-polarized beam. [18] Spin decoherence refers to the depolarization caused by the difference in the beam particles' spin precession frequencies.

The difference in spin tunes is due to the difference of the particles' orbit lengths, and hence their equilibrium energy levels, on which spin tune depends. One way spin decoherence can be suppressed is by utilization of sextupole fields. We consider how this can be accomplished in section 2.2.

\section{Machine imperfections}

As we have seen, the problem with machine imperfections is twofold: (i) they are practically impossible to remove at the present level of technology; but what's even worse, (ii) their removal leaves one in the space domain, and opens the measurement up to geometric phase error.

Fortunately for us, the imperfection spin kicks they induce change sign when the beam circulation direction is reversed. Their magnitude is also sufficient for use as a Koop Wheel. In more detail, the question of the machine imperfection MDM precession is considered in section 2.3.

The one remaining difficulty is the accuracy of the Koop wheel roll direction flipping. This question is considered in section 2.4.

\section{D FS method}

\section{Main features}

The method we propose is characterized by two main features: 
(1) It is a frequency domain method;

(2) The fields induced by machine imperfections, instead of being suppressed, are used as a Koop Wheel.

- The Koop Wheel roll direction is reversed by flipping the direction of the guide field;

- its roll rate is controlled through observation of spin precession in the horizontal plane.

The advantages of the frequency domain, such as (i) ease of polarimetry, and (ii) immunity to geometric phase error, have been discussed in prevous sections. Now we will turn to the description of how machine imperfection fields can be used as a Koop Wheel.

\section{EDM estimator statistic}

Since the angular velocity measured in the frequency domain methodology includes contributions due to both the magnetic and electric dipole moments, the EDM estimator statistic requires two cycles to compose: one in which the Koop Wheel rolls forward, the other backward.

The change in the Koop Wheel roll direction is affected by flipping the direction of the guide field. When this is done: $\boldsymbol{B} \mapsto-\boldsymbol{B}$, the beam circulation direction changes from clockwise (CW) to counter-clockwise $(\mathrm{CCW}): \boldsymbol{\beta} \mapsto-\boldsymbol{\beta}$, while the electrostatic field remains constant: $\boldsymbol{E} \mapsto \boldsymbol{E}$. According to the T-BMT equation, spin precession frequency components change like:

$$
\begin{aligned}
\omega_{x}^{C W} & =\omega_{x}^{M D M, C W}+\omega_{x}^{E D M}, \\
\omega_{x}^{C C W} & =\omega_{x}^{M D M, C C W}+\omega_{x}^{E D M}, \\
\omega_{x}^{M D M, C W} & =-\omega_{x}^{M D M, C C W},
\end{aligned}
$$


and the EDM estimator

$$
\begin{aligned}
\hat{\omega}_{x}^{E D M} & :=\frac{1}{2}\left(\omega_{x}^{C W}+\omega_{x}^{C C W}\right) \\
& =\omega_{x}^{E D M}+\underbrace{\frac{1}{2}\left(\omega_{x}^{M D M, C W}+\omega_{x}^{M D M, C C W}\right)}_{\varepsilon \rightarrow 0} .
\end{aligned}
$$

To keep the systematic error term $\varepsilon$ below required precision, i.e. ensure that equation (1.5a) holds with sufficient accuracy, Y. Senichev devised [20] a guide field flipping procedure based on observation of the beam polarization precession frequency in the horizontal plane.

The idea behind the procedure is outlined in section 2.4; in order for it to make sense, we need to introduce the concept of the effective Lorentz factor (see section 1.2 for that).

\section{Frequency estimation and major statistical properties of the polarimetry data}

The details of our analysis of the polarization precession frequency estimation problem can be found in appendix A. In this section, we will only summarize the main conclusions.

Firstly, the frequency estimate is obtained via fitting a constant-parameter harmonic function to polarimetry data. Since perturbations in the spin dynamics (e.g., due to betatron oscillations) induce a mismatch between the constant-parameter model and the actual data-generating function, regression model specification systematic error is of concern. It is analyzed in section 2.1. According to our results, the 2D FS method is robust to this systematic error.

Secondly, polarimetry data are heteroskedastic, i.e., polarimetry measurement error grows toward the end of the measurement cycle. [24] The Ordinary Least Squares estimation method loses efficiency when fitting such data; the parameter estimate standard errors provided by it become biased and inconsistent. However, the expectation values of the estimates remain valid. Therefore, one can still employ OLS, but together with 
White standard error estimates. But use of heteroskedastic models [25, 26] is still more appropriate.

Thirdly, beam depolarization places stricter constraints on the duration of the measurement cycle, than beam loss. Assume a beam with an infinite lifetime. 3 Obviously, when the beam is fully depolarized, it is impossible to obtain any information about its spin precession rate; i.e. there's a principal bound on the amount of information (denoted $\mathrm{FI}_{\text {tot }}$ ) about the spin precession frequency, which can be gathered from one injection. We will call the period of time during which the polarization drops by a factor of $e$ the polarization lifetime $\tau_{d}$. In Table A.1 we summarized the amount of collected (relative to $\mathrm{FI}_{\text {tot }}$ ) spin precession frequency information as a function of the measurement cycle length, as well as the corresponding signal-to-noise ratio. 4 Going by data from the table, the useful measurement cycle length is limited by three polarization lifetimes.

Fourthly, our simuilations indicate that it is possible to reach a statistical precision of the $8 \cdot 10^{-7} \mathrm{rad} / \mathrm{sec}$ in the frequency estimate in one measurement cycle, assuming the polarization lifetime 1,000 seconds, polarization samling frequency $375 \mathrm{~Hz}$, and initial polarizaton measurement error $3 \%$. At $70 \%$ acclerator duty this is sufficient to reach $5 \cdot 10^{-9} \mathrm{rad} / \mathrm{sec}$ standard error of the mean frequency estimate. Such precision is sufficient for the attainment of an EDM estimate at an uncertainty level of $10^{-29} e \cdot \mathrm{cm}$.

\section{Effective Lorentz factor}

Spin dynamics is described by the concepts of spin tune $\nu_{s}$ and invariant spin axis $\bar{n}$. Spin tune depends on the particle's equilibrium-level energy,

\footnotetext{
${ }^{3}$ Apart from anything else, this implies non-destructive polarimetry.

${ }^{4}$ The ratio is computed accoding to the used polarization signal and measurement error models.
} 
Table 1.1: Amount of gather information (in percents of absolute maximum), as a function of hte measurement cycle, and the corresponding signal-to-noise ratio.

\begin{tabular}{rrr}
\hline Info $\left(\% \mathrm{FI}_{\text {tot }}\right)$ & Cycle length $\left(\times \tau_{d}\right)$ & $\mathrm{SNR}$ \\
\hline 95 & 3.0 & 0.4 \\
90 & 2.3 & 1.1 \\
70 & 1.2 & 5.5 \\
50 & 0.7 & 11.7 \\
\hline
\end{tabular}

expressed by the Lorentz factor:

$$
\left\{\begin{aligned}
\nu_{s}^{B} & =\gamma G \\
\nu_{s}^{E} & =\beta^{2} \gamma\left(\frac{1}{\gamma^{2}-1}-G\right) \\
& =\frac{G+1}{\gamma}-G \gamma .
\end{aligned}\right.
$$

Unfortunately, not all beam particles share the same Lorentz factor. A particle involved in betatron motion will have a longer orbit, and as a direct consequence of the phase stability principle, in an accelerating structure utilizing an RF cavity, its equilibrium energy level must increase. Otherwise it cannot remain the bunch. In this section we analyze how the particle Lorentz factor should be modified when betatron motion, as well as non-linearities in the momentum compaction factor are accounted for.

The longitudinal dynamics of a particle on the reference orbit of a storage ring is described by the system of equations:

$$
\begin{cases}\frac{\mathrm{d}}{\mathrm{d} t} \Delta \varphi & =-\omega_{R F} \eta \delta, \\ \frac{\mathrm{d}}{\mathrm{d} t} \delta & =\frac{q V_{R F} \omega_{R F}}{2 \pi h \beta^{2} E}\left(\sin \varphi-\sin \varphi_{0}\right) .\end{cases}
$$

In the equations above, $\Delta \varphi=\varphi-\varphi_{0}$ and $\delta=\left(p-p_{0}\right) / p_{0}$ are the deviations of the particle's phase and normalized momentum from those of the reference particle; $V_{R F}, \omega_{R F}$ are, respectively, the RF voltage and frequency; $\eta=\alpha_{0}-\gamma^{-2}$ is the slip-factor, where $\alpha_{0}$ is the momentum compaction factor defined by $\Delta L / L=\alpha_{0} \delta, L$ being the orbit length; $h$ is the harmonic number; $E$ the total energy of the particle. 
The solutions of this system form a family of ellipses in the $(\varphi, \delta)$ plane, all centered at the point $\left(\varphi_{0}, \delta_{0}\right)$. However, if one considers a particle involved in betatron oscillations, and uses a higher-order Taylor expansion of the momentum compaction factor $\alpha=\alpha_{0}+\alpha_{1} \delta$, the first equation of the system transforms into: [27, p. 2579]

$$
\begin{aligned}
\frac{\mathrm{d} \Delta \varphi}{\mathrm{d} t}=-\omega_{R F}\left[\left(\frac{\Delta L}{L}\right)_{\beta}\right. & +\left(\alpha_{0}+\gamma^{-2}\right) \delta \\
& \left.+\left(\alpha_{1}-\alpha_{0} \gamma^{-2}+\gamma^{-4}\right) \delta^{2}\right],
\end{aligned}
$$

where $\left(\frac{\Delta L}{L}\right)_{\beta}=\frac{\pi}{2 L}\left[\varepsilon_{x} Q_{x}+\varepsilon_{y} Q_{y}\right]$, is the betatron motion-related orbit lengthening; $\varepsilon_{x}$ and $\varepsilon_{y}$ are the horizontal and vertical beam emittances, and $Q_{x}, Q_{y}$ are the horizontal and vertical tunes.

The solutions of the transformed system are no longer centered at the same single point. Orbit lengthening and momentum deviation cause an equilibrium-level momentum shift [27, p. 2581]

$$
\Delta \delta_{e q}=\frac{\gamma_{0}^{2}}{\gamma_{0}^{2} \alpha_{0}-1}\left[\frac{\delta_{m}^{2}}{2}\left(\alpha_{1}-\alpha_{0} \gamma^{-2}+\gamma_{0}^{-4}\right)+\left(\frac{\Delta L}{L}\right)_{\beta}\right],
$$

where $\delta_{m}$ is the amplitude of synchrotron oscillations, and

$$
\left(\frac{\Delta L}{L}\right)_{\beta}=\frac{\pi}{2 L}\left[\varepsilon_{x} Q_{x}+\varepsilon_{y} Q_{y}\right]
$$

is the betatron motion-related orbit lengthening. $\varepsilon_{x}$ and $\varepsilon_{y}$ are, respectively, the horizontal and vertical beam emittances, $Q_{x}$ and $Q_{y}$ are the horizontal and vertical betatron tunes.

We call the equilibrium energy level associated with the momentum shift (1.8), the effective Lorentz factor:

$$
\gamma_{e f f}=\gamma_{0}+\beta_{0}^{2} \gamma_{0} \cdot \Delta \delta_{e q}
$$

where $\gamma_{0}, \beta_{0}$ are the Lorentz factor and relative velocity factor of the reference particle. 
Observe, that the effective Lorentz factor enables us to account for variation in the value of spin tune due to variation in the particle orbit length. It is crucial in the analysis of spin decoherence (see section 2.2) and its suppression by means of sextupole fields.

It plays a big role, as well, in the successfull reproduction of the MDM component to the total spin precession angular velocity.For that reason, we would like to refer the reader to section 2.5.

\subsection{Frozen- and Quasi-frozen spin lattice}

There exist two design approaches to the problem of measuring the deuteron EDM inside a storage ring: (i) the Frozen Spin (FS) lattice, and (ii) the Quasi-frozen spin (QFS) lattice.

In the following sections we will consider variants of both type lattices.

\section{The Frozen Spin lattice}

In an FS-type lattice, the horizontal projection of a beam particle's spin vector is continuously aligned with its momentum vector. In order to realize the continuity condition, combined $\mathrm{E}+\mathrm{B}$-field cylindrical spin-rotators are inserted into the accelerator arc sections. An example of a FS-type lattice [28] is shown in Figure 1.1. This ring is $145.85 \mathrm{~m}$ in length and is designed for the deuteron beam injection energy $270 \mathrm{MeV}$. An RF cavity is used in order to suppress linear spin decoherence effects by timeaveraging the beam particles' kinetic energies. The RF voltge used is $V=100 \mathrm{kV}$, RF frequency $f_{R F}=5 \cdot f_{\text {rev }}$, where the cyclotron frequency $f_{\text {rev }}=1.00 \mathrm{MHz}$. The remaining non-linear decoherence effects are suppressed by means of three ${ }^{5}$ sextupole families.

The main purpose of the FS lattice design is to maximize the EDM signature signal. However, it is important to note that, strictly speaking, the FS condition is fulfilled only for the reference particle. This is because, as follows from equation (1.1b), for any given E- and B-fields there exists

\footnotetext{
${ }^{5}$ Some authors use two families [18] in this lattice.
} 


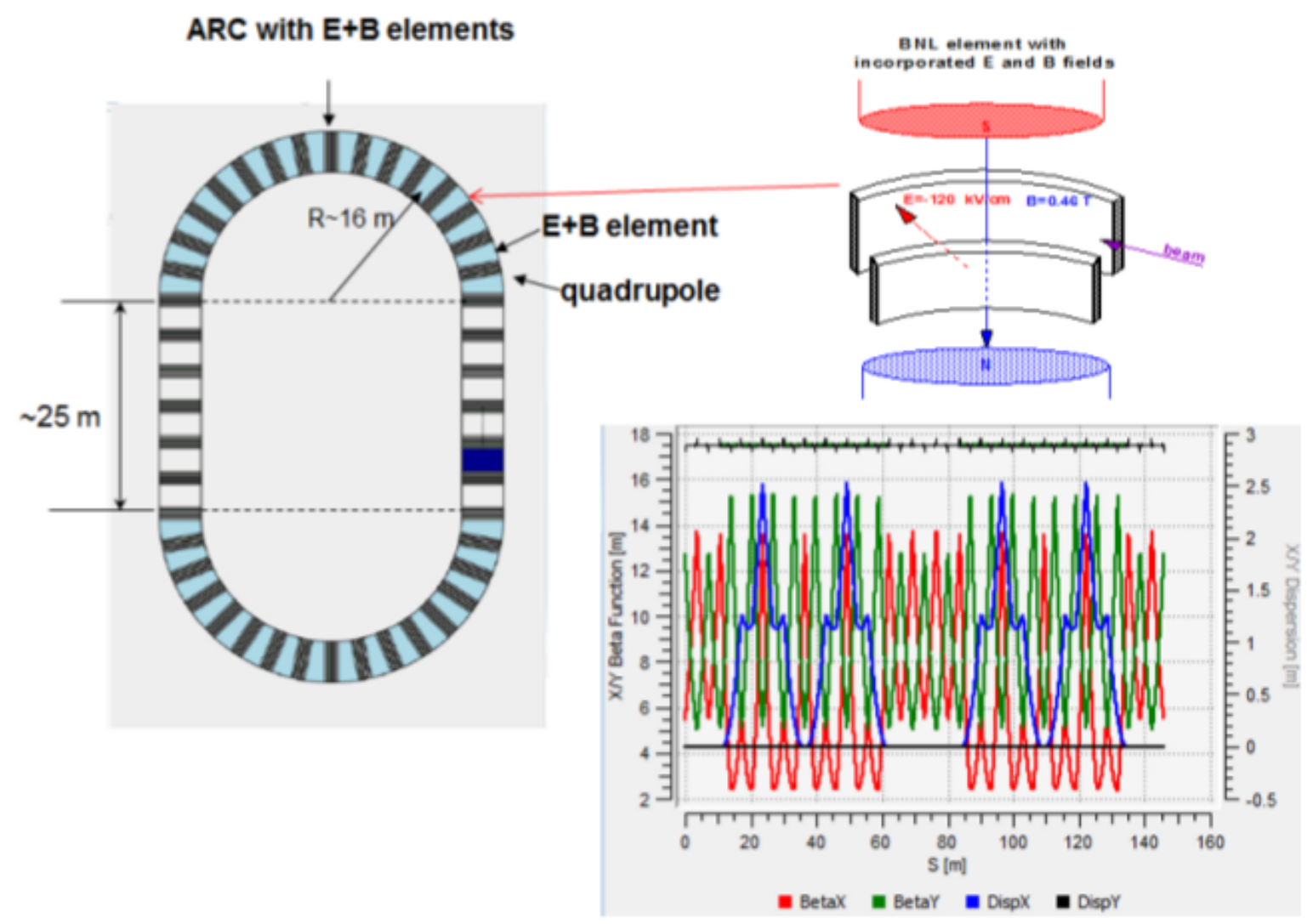

Figure 1.1: A FS lattice variant. Cylindrical E+B spin-rotators are used in the arc sections to fulfill the continuous FS condition. (Image is taken from [28].)

a unique value of the Lorentz factor $\gamma$ at which $\Omega_{y}^{M D M}=0$. Hence, even in a FS lattice, most particles' spin vectors are frozen only approximately.

\section{Quasi-Frozen Spin lattice}

In the QFS design concept, one gives up the continuity property of the FS state, requiring only that the spin phase advance (in the rest frame) in the electrostatic $\left(\Phi_{s}^{E}\right)$ and magnetic $\left(\Phi_{s}^{B}\right)$ elements was zero on average 
(at each turn): [28]

$$
\sum_{i} \Phi_{s, i}^{E}=-\sum_{j} \Phi_{s, j}^{B}
$$

Following the definition of spin tune (see section 1.1), a particle's spin vector placed into an electromagnetic field turns by angle $\Phi_{s}=\nu_{s} \cdot \Phi$, where $\Phi$ is the momentum angle of turn, $\nu_{s}$ spin tune.

A particle's angular momentum, when placed into a magnetic field $\boldsymbol{B}$ is

$$
\omega_{B}=\frac{q}{m} \frac{B}{\gamma}
$$

into an electrostatic $\boldsymbol{E}$ :

$$
\omega_{E}=\frac{q}{E} \frac{\boldsymbol{E} \times \boldsymbol{\beta}}{c \beta^{2} \gamma}
$$

from which follow the expressions for spin tune in the electrostatic and magnetic fields:

$$
\left\{\begin{array}{l}
\nu_{s}^{B}=\gamma G, \\
\nu_{s}^{E}=\beta^{2} \gamma\left(\frac{1}{\gamma^{2}-1}-G\right) .
\end{array}\right.
$$

The QFS lattice design has the advantage of simplicity over the FS one: there's no need to use a combined-field cylindrical spin rotators; in both QFS lattice variants we consider below are used either (i) straight Wien filters, or (ii) cylindrical electrostatic and magnetic elements separately. On the other hand, due to the appearance of a vertical spin precession axis component $\bar{n}_{y}$, the maximum EDM signal amplitude is less compared with the pure FS case. The attenuation factor [29]

$$
J_{0}\left(\Phi_{s}\right) \approx 1-\frac{\Phi_{s}^{2}}{4}
$$

where $\Phi_{s}$ is the maximum horizontal plane spin phase advance.

Assume the phase advance does not exceed $\pi \cdot \gamma G / 2 n$; in this context $n$ is the lattice periodicity. Since the deuteron anomalous magnetic moment $G=-0.142, J_{0} \geq 0.98$ for the QFS lattices considered below. 


\section{QFS lattice design "6.3"}

A QFS design lattice [28] in which E- and B-fields are separated in space is presented in Figure 1.2. Negative radius electrostatic cylindrical deflectors are used to compensate the spin phase advance related to the MDM precession in the arc sections. [29] The ring is $166.67 \mathrm{~m}$ length long and is designed for $270 \mathrm{MeV}$ injection energy. For the suppression of linear spin decoherence effects, an RF cavity is used, with voltage $V=100 \mathrm{kV}$, and operating frequency $f_{R F}=5 \cdot f_{\text {rev }}$, where $f_{\text {rev }}=0.87 \mathrm{MHz}$. Non-linear decoherence effects are suppressed means of six sextupole families.

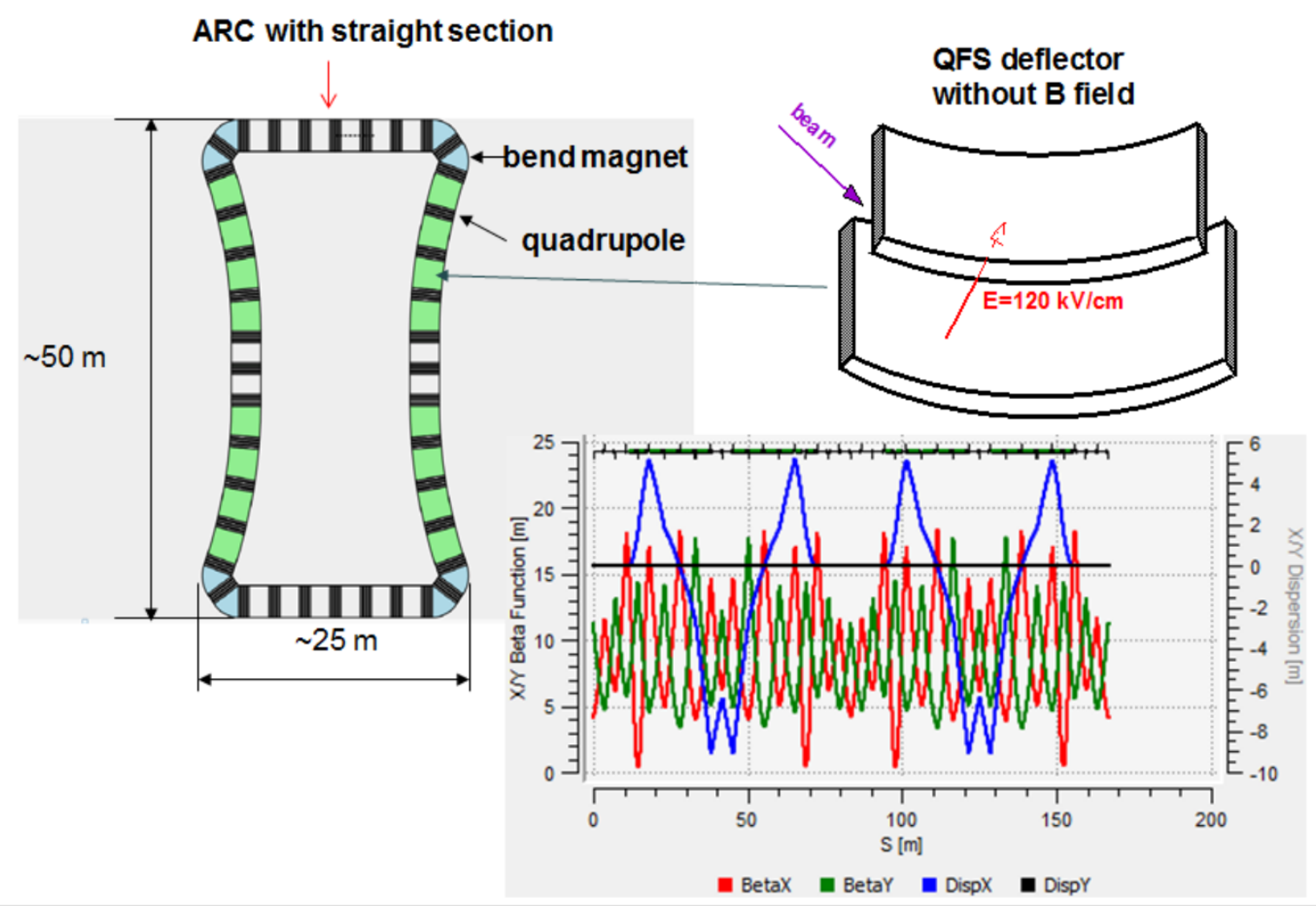

Figure 1.2: QFS lattice design variant with spatially separated E- and B-fields. (Image taken from [28]) 


\section{QFS lattice design "E+B"}

The lattice design in Figure 1.3 utilizes plain, straight, static Wien filters. This allows one to: a) exclude non-linear electrostatic field components present in curved electrostatic fields, and $b$ ) simplify the lattice from the engineering point of view.

The lattice is $149.21 \mathrm{~m}$ in length, the injection energy is $270 \mathrm{MeV}$. The linear spin decoherence effects suppressing RF cavity has a longitudinal voltage $V=100 \mathrm{kV}$, and frequency $f_{R F}=5 \cdot f_{\text {rev }}$, with $f_{\text {rev }}=0.98 \mathrm{MHz}$. Four sextupole families are used in the suppression of non-linear decoherence effects.

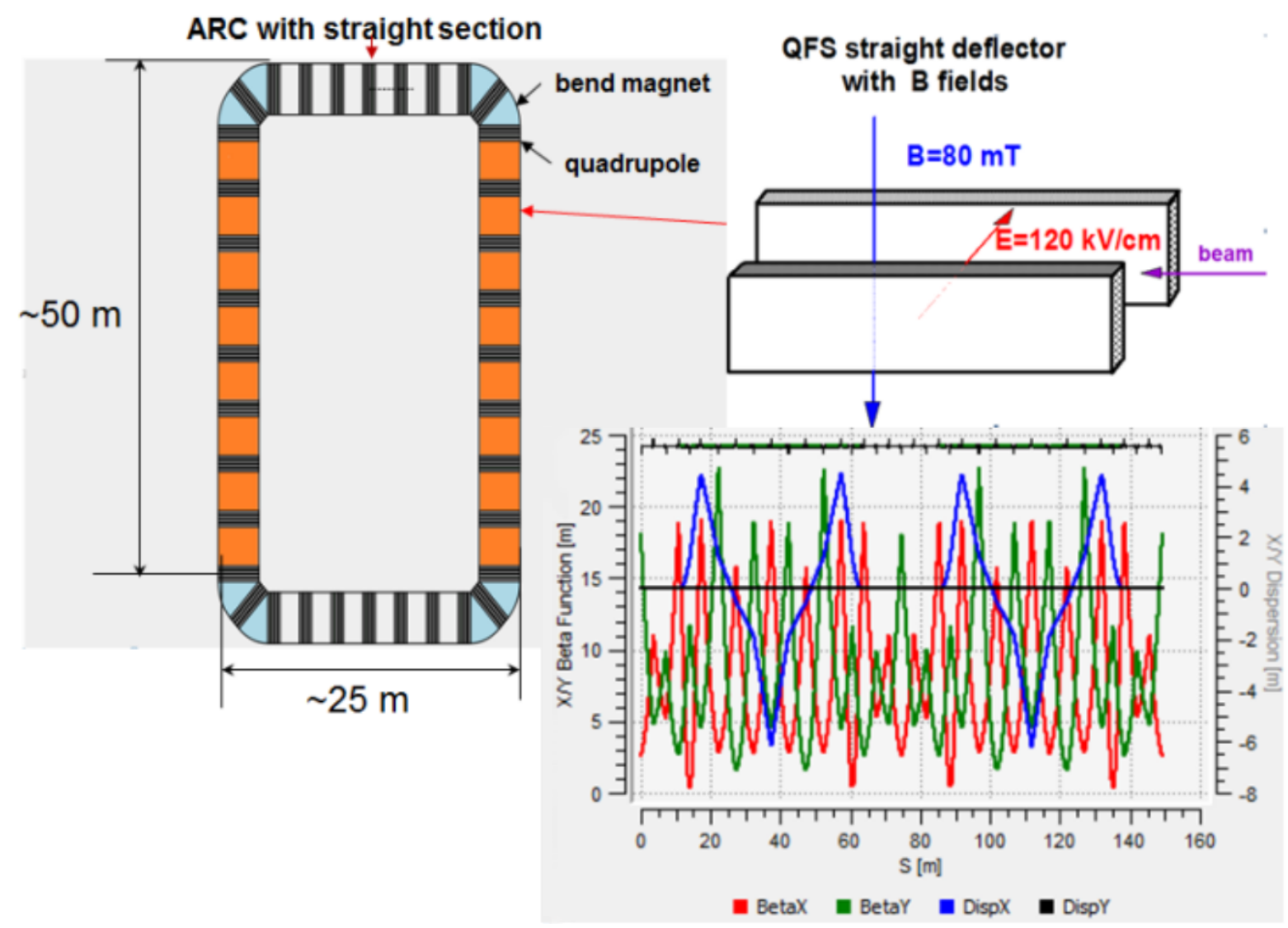

Figure 1.3: Straight Wien filters QFS lattice variant. (Image taken from [28]) 


\section{Chapter 2}

\section{Universal SR EDM measurement problems and their solutions}

Universal SR EDM measurement problems can be classified into two groups: (i) problems that can ba solved by introducing a spin wheel, and (ii) problems needing specialized solutions.

Problems of the first category follow from the instability of the invariant spin axis. Among those are, for example, local electromagnetic field perturbaitons, as well as perturbations to the particle spin dynamics caused by betatron oscillations. In both cases the particle invariant spin axis deviates from is equilibrium (closed orbit) orientation for a short period of time.

Problems needing specific solutions include spin decoherence and EDMfaking MDM spin precession. In this part we analyze the essence of each of these problems, describe their possible solutions, and perform corresponding simulations.

\subsection{Perturbations to the spin dynamics}

\section{Problem statement}

The invariant spin axis of a particle involved in betatron oscillations wobbles about its reference orientation. [30, p. 11] For this reason, the amplitude of the T-BMT equation solution for the vertical spin vector compo- 
CHAPTER 2. UNIVERSAL SR EDM MEASUREMENT PROBLEMS AND THEIR SOLUTIONS

nent:

$$
\begin{aligned}
s_{y} & =\sqrt{\left(\frac{\omega_{y} \omega_{z}}{\omega}\right)^{2}+\left(\frac{\omega_{x}}{\omega}\right)^{2}} \cdot \sin (\omega \cdot t+\phi) \\
& =\sqrt{\left(\bar{n}_{y} \bar{n}_{z}\right)^{2}+\bar{n}_{x}^{2}} \cdot \sin \left(2 \pi \cdot \nu_{s} \cdot n_{\text {turn }}+\phi\right),
\end{aligned}
$$

becomes a time-varying function. If a particle's invariant spin axis (as well sa spin tune) varies in a sufficiently big range, use of a constant parameter harmonic function as a model for fitting the measured signal will introduce the model specification systematic error. Errors of this type reflect on the validity of the model parameter estimates, i.e. the frequency estimate, and hence require analysis.

Spin tune variability is especially problematic in this respect, since it directly affects the phase of the signal; however, this problem can be solved by introducing sextupole field elements into the beamlinr, as is described in section 2.2. For this reason, we will focus on the variation of $\bar{n}$ in this section.

\section{Simulation}

The simulation setup was as follows: a particle offset from the reference orbit in the vertical direction by $0.3 \mathrm{~mm}$, is injected multiple times into an imperfect FS-type lattice [28], in which we suppress spin decoherence caused by vertical plane betatron oscillations (see section 2.2) by using the corresponding sextupole family. Machine imperfections are simulated as $\mathrm{E}+\mathrm{B}$ element tilts about the optic axis. Imperfections introduced this ways do not perturb the closed orbit (that is, the reference orbit - as well as the orbit of the betatron-oscillating particle — is the same for every injection.)

Each trial, $\mathrm{E}+\mathrm{B}$ element tilts are randomly distributed as $\alpha \sim N\left(\mu_{i}, 3\right.$. $10^{-4}$ ) degrees, $i \in\{1, \ldots, 11\}$, where $\mu_{i}$ varies in the range $\left[-1.5 \cdot 10^{-4},+2.5\right.$. $\left.10^{-4}\right]$ degrees. Non-zero expectation $\mu_{i}$ simulated the introduction of a spin wheel driver into the beamline. [22] Magnitudes of $\mu_{i}$ and $\sigma_{\alpha}$ were picked 
CHAPTER 2. UNIVERSAL SR EDM MEASUREMENT PROBLEMS AND THEIR SOLUTIONS

for better detalization of the effect. At bigger values, it is more difficult to distinguish the $\nu_{s}$ and $\bar{n}$ variation effects.

Another aspect of the simulation worth mentioning is that the particle injection energy of $270 \mathrm{MeV}$, which is not exactly the FS energy for this lattice (270.0092 MeV is the most precise value we could obtain). Because of this the invariant spin axis $\bar{n}$ points mostly in the vertical direction (deviating from it by no more than $51^{\circ}$ at higher spin wheel roll rates); its radial component (determining the spin vector's vertical component's oscillation amplitude) is relatively small, and hence the more sensitive to perturbations caused by the betatron motion.

Spin tracking was done in COSY Infinity [31], for $1.2 \cdot 10^{6}$ beam revolutions; every 800 revolutions $\nu_{s}$ and $\bar{n}$ were computed (using procedure TSS [32, p. 41]) at the phase space point occupied by the particle at the moment, which gives us the first data set $\left(\nu_{s}(n), \bar{n}(n)\right), n$ being the revolution number. The corresponding spin vector components $\left(s_{x}^{\text {trk }}(n), s_{y}^{\text {trk }}(n), s_{z}^{\text {trk }}(n)\right)$, computed by the tracker (procedure TR [32, p. 41]), make up the second set of data series used in the analysis.

\section{Analysis}

Using the first data set we computed the expected $s_{y}^{g e n}(t)$ "generator" time series, according to equation (2.1), as well as the "ideal" series $s_{y}^{i d l}$, in which we assumed constant values $\nu_{s}=\left\langle\nu_{s}(t)\right\rangle$ and $\bar{n}=\langle\bar{n}(t)\rangle$.

Our hypothesis is that the betatron motion will introduce a discrepancy between the ideal harmonic model

$$
f(t)=a \cdot \sin (\omega \cdot t+\delta),
$$

and the tracker data, by varying the spin precession axis $\bar{n}$, and hence the amplitude of the fitted signal. The "ideal" series serves as the basis for analysis, since it perfectly corresponds to the regression model; the "generator" series accounts for the variation of $\bar{n}$, while still remaining within the bounds of the regression model. The "tracker" series is our closest approximation to the real measurement data. 
CHAPTER 2. UNIVERSAL SR EDM MEASUREMENT PROBLEMS AND THEIR SOLUTIONS

In order to cross-compare the series, we a) computed and analyzed the residuals $\epsilon_{1}(t)=s_{y}^{g e n}(t)-s_{y}^{i d l}(t)$ and $\left.\epsilon_{2}(t)=s_{y}^{t r k}(t)-s_{y}^{i d l}(t) ; b\right)$ fitted model (2.2) to the three time series and compared the fit quality; $c$ ) computed the standard deviations of the $\bar{n}$ components at different spin wheel roll rates.
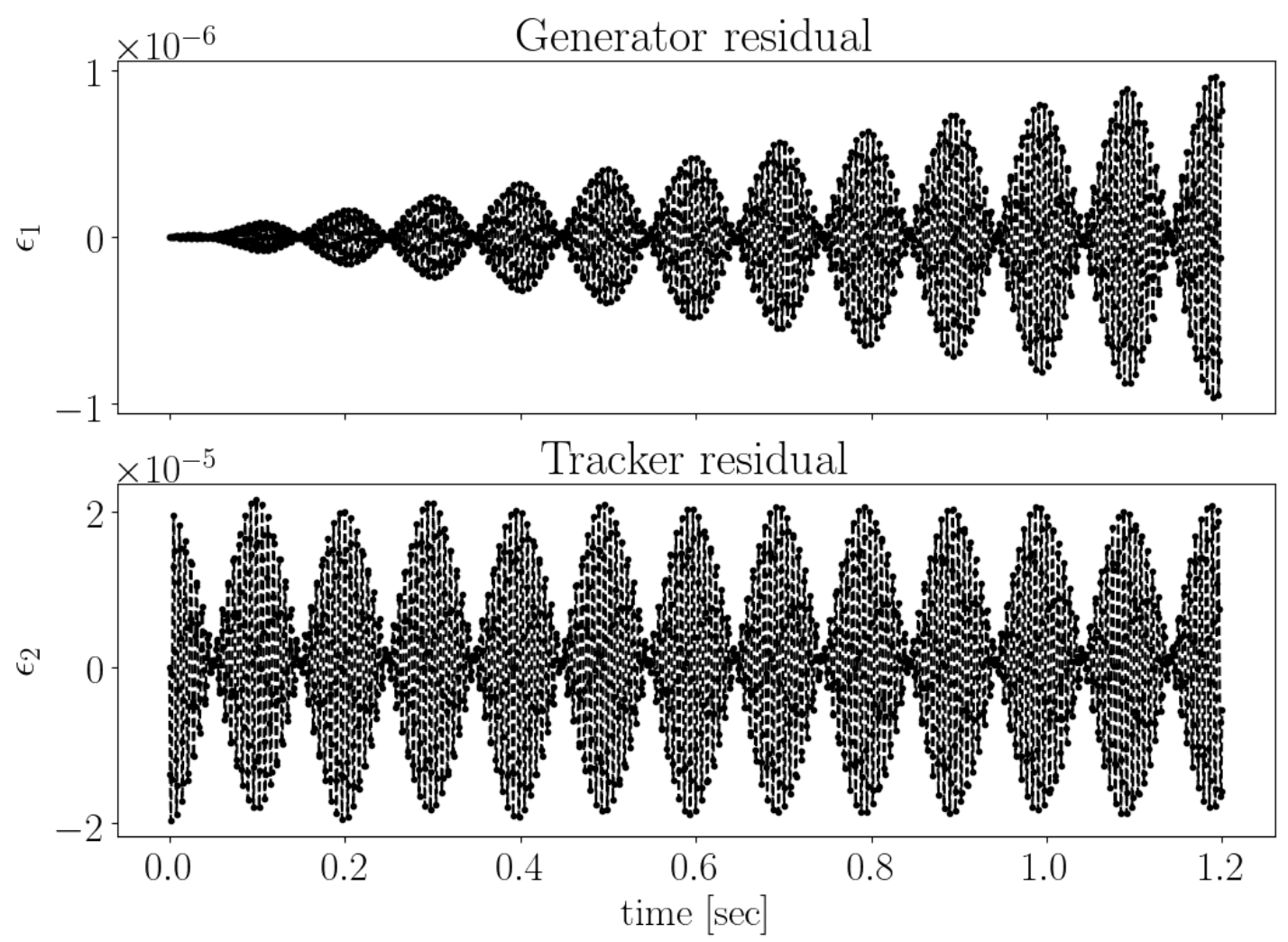

Figure 2.1: Comparator residuals as functions of time. Top panel: $\epsilon_{1}$ residual; bottom panel: $\epsilon_{2}$ residual

In Figure 2.1 we observe that the "generator" is almost identical to the "ideal" series, with $\epsilon_{1} \leq 1 \cdot 10^{-6}$ (even though its oscillation frequency is slightly off) for the duration of the cycle, while the "tracker" series deviates from it at the level $\epsilon_{2} \leq 2 \cdot 10^{-5}$. The discrepancy betweem $\epsilon_{1}$ and $\epsilon_{2}$ is observed systematically at all spin wheel roll rates (see Figure 2.2b), and does not have an explanation so far.

In Figure $2.2 \mathrm{~b}$ we see that the standard deviations of both residuals exhibit the same dependence on the spin wheel roll rate as that of $\nu_{s}$ 
CHAPTER 2. UNIVERSAL SR EDM MEASUREMENT PROBLEMS AND THEIR SOLUTIONS

Table 2.1: Model parameter estimates (slow SW)

\begin{tabular}{c|rllr}
\hline Series & Par. & Value & St.Err & AIC \\
\hline \multirow{3}{*}{$s_{y}^{\text {idl }}$} & $\hat{f}$ & 4.220359687911 & $6.9 \cdot 10^{-11}$ & \\
& $\hat{a}$ & 0.12514597851 & $4 \cdot 10^{-11}$ & -62093 \\
& $\hat{\delta}$ & $-1.50 \cdot 10^{-8}$ & $4 \cdot 10^{-10}$ & \\
\hline \multirow{3}{*}{$s_{y}^{\text {gen }}$} & $\hat{f}$ & 4.2203596911 & $1.9 \cdot 10^{-9}$ & \\
& $\hat{a}$ & 0.125145979 & $1 \cdot 10^{-9}$ & -52142 \\
& $\hat{\delta}$ & $-1.6 \cdot 10^{-8}$ & $1.2 \cdot 10^{-8}$ & \\
\hline \multirow{3}{*}{$s_{y}^{\text {trk }}$} & $\hat{f}$ & 4.2203603 & $1.3 \cdot 10^{-6}$ & \\
& $\hat{a}$ & 0.12514597 & $3.7 \cdot 10^{-7}$ & -34567 \\
& $\hat{\delta}$ & $-4 \cdot 10^{-6}$ & $6 \cdot 10^{-6}$ & \\
\hline
\end{tabular}

(Figure 2.2a, bottom panel), but show indifference toward the behavior of $\bar{n}$. This is an indication that frequency variation contributes a great deal more to the discrepance between model (2.2) and the tracker data than the presumed amplitude variation caused by the wobbling of $\bar{n}$ during betatron oscillations.

Table 2.1 characterized the model fit quality with respect to the used data set at the slowest spin wheel roll rate. We observe that the crossdifferences between the parameter estimates at different time series are not statistically significant. Even though the variation of the spin precession angular velocity dagraded the fit quality, it did not introduce any statistically-significant bias into the estimates.

\section{Conclusions}

The question of the influence of betatron motion on the EDM statistic in the FD method should be considered in view of three circumstances:

(1) The signal amplitude oscillations (as estimated by $\epsilon_{2}$ ) are small. They occur at the $10^{-4}$ level (when $\alpha \sim N\left(0,3 \cdot 10^{-2}\right)$ degrees), whereas the expected polarization measurement error is on the order of percents. This means the superposition of this systematic er- 
CHAPTER 2. UNIVERSAL SR EDM MEASUREMENT PROBLEMS AND THEIR SOLUTIONS

ror with the random measurement error will exhibit no statisticallysignificant systematicity.

(2) The correllation coefficient between the amplitude and frequency estimates is not significant. The amplitude oscillations affect the $\hat{a}-$ estimate foremost; their effect on the $\hat{\omega}$-estimate is secondary, and is described by the correlation coefficient. Since it is less than $10 \%$, even if the oscillations happen to be strong enough to affect the amplitude estimate, their effect on the frequency estimate will be reduced by at least a factor of 10 .

(3) This systematic effect is controllable. And this point is the major advantage of the FD methodology. By applying an external Spin Wheel, the $\bar{n}$ oscillations can be continuously minimized as much as necessary, without changing the experiment pattern.

\section{$2.2 \quad$ Spin decoherence}

Spin coherence refers to a measure or quality of preservation of polarizaion in an initially fully-polarized beam. [18, p. 205]

The spin vectors of a polarized beam injected into a storage ring begin precessing about the vertical (guiding) field. The precession frequency depends on the particle equilibrium energy level, which differs across the beam particles.

This circumstance doesn't pose a problem when the beam is vertically polarized; however, the FS SR EDM measurement method requires that the polarization vector be aligned with the beam's momentum vector, i.e. lay in the horizontal plane. Hence, spin decoherence is an inherent problem of the FS methodology.

In the present section we analyze the origins of spin decoherence, the sextupole method of its suppression, as well as the simulation results proving the effectiveness of the method. 
CHAPTER 2. UNIVERSAL SR EDM MEASUREMENT PROBLEMS AND THEIR SOLUTIONS

As an introduction, though, we estimate the spin coherence time required for the measurement of the EDM in the framework of the space domain methodology.

\section{Spin coherence time requirements}

Operating in the space domain FS methodological framework in a perfectlyaligned lattice, 11 the spin coherence time (SCT) is determined by the minimal detectable angle by which the polarization vector deviates from the beam orbit plane as a result of the EDM precession alone. For the sensitivity level of $10^{-29} \mathrm{e} \cdot \mathrm{cm}$ this angle is approximately $5 \cdot 10^{-6}$. [12]

According to the T-BMT equation,

$$
\Omega_{E D M, x}=\eta \frac{q E_{x}}{2 m c},
$$

where $\eta$ is the proportionality coefficient between the EDM and spin, in the deuteron case equal to $10^{-15}$, for the given sensitivity level. [18, p. 206]

For the deuteron BNL FS ring, $E_{x}=12 \mathrm{MV} / \mathrm{m}$, [12, p. 19] therefore $\Omega_{E D M, x} \approx 10^{-9} \mathrm{rad} / \mathrm{sec}$. Hence we obtain that, in order to reach a detectable level of at least $1 \mu \mathrm{rad}$ one needs an SCT on the order of 1,000 seconds. [18, p. 207]

\section{Origins of decoherence}

Spin decoherence in a particle beam is caused by the difference between the beam particles' spin precession angular velocities, which is, in turn, a result of the difference between their orbit lengths and initial momenta. Spin tune dispersion can be described by the concept of the effective Lorentzfactor, which was introduced in section 1.2 .

From equations (1.6) for spin tune in electrostatic and magnetic fields it follows that the spin tunes of two particles having equal values of the effective L-factor are equal, regardless of their trajectories in the accelerator. This principle is the basis for the proposed sextupole field spin

\footnotetext{
${ }^{1}$ In fact, perfect element alignment is a pre-requirement of the space domain.
} 
CHAPTER 2. UNIVERSAL SR EDM MEASUREMENT PROBLEMS AND THEIR SOLUTIONS

decoherence suppression theory, as well as the procedure for flipping the polarity of the storage ring's guide field, which is required for injecting the deuteron beam in the opposite direction in order to cancel the EDM-faking MDM spin precession.

\section{Sextupole field spin decoherence suppression theory}

In order to minimize spin decoherence related to particle betatron motion and momentum spread, sextupole (or octupole) fields can be used. [18, p. 212]

A sextupole of strength

$$
S_{\text {sext }}=\frac{1}{B \rho} \frac{\partial^{2} B_{y}}{\partial x^{2}},
$$

(where $B \rho$ is the magnetic rigidity) modifies the first-order momentum compaction factor as [27, p. 2581]

$$
\Delta \alpha_{1, \text { sext }}=-\frac{S_{\text {sext }} D_{0}^{3}}{L}
$$

and simultaneously the orbit length as

$$
\left(\frac{\Delta L}{L}\right)_{\text {sext }}=\mp \frac{S_{\text {sext }} D_{0} \beta_{x, y} \varepsilon_{x, y}}{L},
$$

where $D(s, \delta)=D_{0}(s)+D_{1}(s) \delta$ denotes the dispersion function.

One can formulate the principle of the sextupole field effect in the following way. A particle in an accelerator does betatron oscillations about some closed orbit. Due to dispersion, the closed orbit is different for different particles in the beam. 2 A sextupole field works like a prism, focusing (or defocusing) the particles' closed orbits.

In the next sections we will denote the decoherence associated with horizontal/vertical betatron oscillations, respectively synchrotron oscillations, X-/Y-, and D-decoherence. Sextupole families aimed at reducing X-, Y-, and D-decoherence will be denoted, respectively, GSX, GSY, GSD.

\footnotetext{
${ }^{2}$ Each closed orbit corresponds to a different equilibrium energy level
} 
CHAPTER 2. UNIVERSAL SR EDM MEASUREMENT PROBLEMS AND THEIR SOLUTIONS

From equations (2.3), and (2.4) one can see that one needs to use three sextupole families, placed respectively in the maxima of the $\beta_{x}, \beta_{y}$ (for the $\mathrm{X}$-, Y-types), and $D_{0}$ (for the D-type) functions, in order to suppress spin decoherence in the beam.

\section{Simulation in an ideal ring}

In order to check the capability of the sextupole field spin decoherence suppression method we carried out a simulation in which we used the FStype lattice described in section 1.3. Since the lattice is perfectly aligned, spin precession occurs only about the vertical $(\hat{y})$ axis.

SCT optimization is done at $270.00 \mathrm{MeV}$ energy, the orbital and spin transfer maps of the lattice are computed up to the fifth order in the Taylor expansion.

Three sextupole families are used, suppressing the X, Y-, and D-type decohrence respectively. Each sextupole family's field gradient is optimized separately (the gradients of the other two families are set to zero). We optimize the sextupoles separately because otherwise we run into a numerical problem with the TSS procedure. $3^{3}$

The sextupole gradient optimization procedure is as follows. First, the lattice's transfer maps are computed at the given value of gradient. Then, using TSS, we compute the spin tune and invariant spin axis (ISA) Taylor expansions over phase space

$$
\nu_{s}(x, a, y, b, \ell, \delta)=a_{0}+a_{1, x} x+a_{2, x} x^{2}+a_{1, y} y+\ldots
$$

Depending on the sextupole family being optimized, we pick the coefficient at the square of the corresponding phase space variable $\left(a_{2, x}, a_{2, y}\right.$, or $\left.a_{2, \delta}\right)$ from the spin tune Taylor expansion. The absolute value of the coefficient

\footnotetext{
${ }^{3}$ We also studied the possibility of finding the optimal set of gradient values, by directly computing the relevant spin tune Taylor expansion coefficients in the 3D gradient space mesh. The question needs further investigation, but at this point we doubt that all three families can be optimized simultaneously. This could be the reason why in [18, p. 219] only two sextupole families are used in the lattice codenamed BNL.
} 
CHAPTER 2. UNIVERSAL SR EDM MEASUREMENT PROBLEMS AND THEIR SOLUTIONS

is used as the objective function: i.e., at the optimal gradient, spin tune does not depend (parabolically) on the corresponding phase space variable.

The Simplex algorithm was used for optimization. [33, p. 37]

In Figure 2.3 we plotted the spin tune dependence on the particle phase space coordinate before and after turning on the relevant sextupoles. One can see that in all three cases the parabolic dependence has been suppressed. However, there remains a linear term, which is insensitive to the sextupole fields. The linear term is observed when modeling the spin dynamics in tree different simulation software: COSY INFINITY, MODE, and also MAD (from private communication with Y. Senichev). Based on that, one can hypothesize that the linear term is not a numerical artifact of COSY INFINITY, but rather has a physical basis. This question needs further analysis, but at this point it is thought that this term can be suppressed by adjusting the RF cavity parameters. [18, p. 210, 219]

\section{Transfer of decoherence into the vertical plane in an imperfect lattice}

The purpose of this simulation is to show that the sextupole approach to the suppression of spin decoherence is agnostic to spatial orientation.

We injected an ensemble of 30 particles, uniformly distributed along the vertical axis in the range $y \in[-1,+1] \mathrm{mm}$, into an imperfect FS lattice. Since the analysis is based only on the tracker data, and does not involve the TSS procedure [32], the beam was injected at the strict FS energy $270.0092 \mathrm{MeV}$.

Imperfections are simulated as $\mathrm{E}+\mathrm{B}$ element tilts about the optic axis by angles picked from the normal distribution $\Theta_{\text {tilt }} \sim N\left(0,1 \cdot 10^{-4}\right)$ radians. Since such imperfections preserve the Lorentz force, they do not perturb the particle orbital dynamics (i.e., the closed orbit) and affect only the spin dynamics. The magnitude of the standard deviation reflects the realistic element alignment precision.

In Figure 2.4 we show the standard deviation of the ensemble spin vector radial component distribution before and after turning on the sex- 
CHAPTER 2. UNIVERSAL SR EDM MEASUREMENT PROBLEMS AND THEIR SOLUTIONS

tupoles. Since the particles move in an imperfect lattice, their spin vectors rapidly turn about the radial axis. This makes $\sigma_{s_{x}}$ a rapidly oscillating function exhibiting no long-term growth trend (the slope of the trend line is $\left.(2 \pm 2) \cdot 10^{-8} 1 / \mathrm{sec}\right)$. This means there's no spin decoherence in the horizontal plane. When the sextupoles are turned on, the $\sigma_{s_{x}}$ amplitude is reduced by a factor of 10 .

In Figure 2.5 the same statistic is shown for the vertical spin vector components. A long-term trend is observed (the slope is $(4.5 \pm 0.6)$. $10^{-7} 1 / \mathrm{sec}$ ) prior to turning on the correcting sextupoles. The sextupole correction does not reduce the oscillation amplitude, but suppresses the accummulation of dispersion (the slope drops to $\left.(5 \pm 6) \cdot 10^{-8} 1 / \mathrm{sec}\right)$.

\section{Analysis of spin decoherence in an imperfect lattice}

The following tests were done with a planar bunch of 30 particles injected into a FS lattice with $\mathrm{E}+\mathrm{B}$ elements tilted about the optic axis by angles picked from $N\left(0,5 \cdot 10^{-4}\right)$ radians.

The particles were normally-distributed in the vertical plane $y-z$ along the $\hat{y}$-axis as in $y \sim N\left(y_{0}, 0.1\right) \mathrm{mm}$ (all other phase space coordinates are zero). The offset $y_{0}$ varied in the range $[-1,+1] \mathrm{mm}$. Initially all spins in the ensemble were longitudinally oriented: $\boldsymbol{S}(t=0)=(0,0,1)$.

We also varied the gradient $G_{Y}$ of the GSY sextupole. $G_{Y}$ varied in the range $\left[G_{Y}^{0}-5 \cdot 10^{-3}, G_{Y}^{0}+5 \cdot 10^{-3}\right]$, where $G_{Y}^{0}=-5.77 \cdot 10^{-4}$ is the optimal gradient for this particular imperfection distribution. The value $G_{Y}^{0}$ was found by minimizing the coefficient $a_{2}$ of the Taylor expansion $\nu_{s}(y) \approx a_{0}+a_{1} \cdot y+a_{2} \cdot y^{2}+O\left(y^{3}\right)$.

There were 10 injections per each value of $G_{Y}$.

Since the TSS procedure was used, to ensure its stability, the beam was injected at $270 \mathrm{MeV}$ (as opposed to the strict FS $270.0092 \mathrm{MeV}$ ), and the orbital and spin transfer maps were computed up to the third order Taylor expansion.

After that, the beam is tracked through the lattice for $1.2 \cdot 10^{6}$ turns, which is approximately equivalent to 1.2 seconds. Data used in the analysis were collected every 800 turns. 
CHAPTER 2. UNIVERSAL SR EDM MEASUREMENT PROBLEMS AND THEIR SOLUTIONS

What we collected: (a) TSS procedure results: spin tune and the ISA components, and (b) spin $\left(S_{X}, S_{Y}, S_{Z}\right)$ and phase space $(X, A, Y, B, T, D)$ vector components. We also recorded the Taylor expansions of $\nu_{s}, \bar{n}$, orbital, and spin transfer maps of the lattice at each $G_{Y}$ value.

Based on the spin vector component data we computed the ensemble polarization:

$$
\boldsymbol{P}=\frac{\sum_{i} \boldsymbol{s}_{i}}{\left|\sum_{i} \boldsymbol{s}_{i}\right|}
$$

Its vertical component is fitted by $f(t ; a, f, \phi)=a \cdot \sin (2 \pi \cdot f \cdot t+\phi)$, where all three parameters $(\hat{a}, \hat{f}, \hat{\phi})$ are estimated.

\section{Sextupole field effect on spin tune and invariant spin axis}

In Figure 2.6 we showed the dependence of spin tune on the particle's vertical offset from the reference orbit: $\nu_{s}(y) \approx a_{0}+a_{1} \cdot y+a_{2} \cdot y^{2}+O\left(y^{3}\right)$. In Figure 2.6a one can observe the unbending of the parabola when $G_{Y} \rightarrow$ $G_{Y}^{0}$.

An equivalent dependence for the vetrical component of the ISA is shown in Figure 2.7. In Figure 2.7a we observe that the ISA component behaves the same way as spin tune when $G_{Y} \rightarrow G_{Y}^{0}$. Just as in the case of an ideal lattice, in Figure 2.7b one can observe the presence of a linear term in $\bar{n}_{y}(y)$, insensitive to the sextupole fields.

In the figures above, the values of spin tune and ISA were computed as univariate functions of the vertical offset; all other phase space coordinates were set to reference values. While analyzing the tracker data we noted that the ISA components (as well as spin tune) of a particle do not oscillate, as one would expect from the figures, but remain nearly constant. We hypothesized that the $\nu_{s}$ and $\bar{n}$ dependencies on the vertical offset and its derivative $\left(y^{\prime} \equiv a\right)$ compensate each other when the particle moves along a real trajectory. In the next figures we depicted $\nu_{s}, \bar{n}$ at their true phase space trajectories in the storage ring.

In Figure 2.8 are depicted the particle trajectories in the $(Y, B)$ phase plane, obtained in tracking the particles through the imperfect lattice. 
CHAPTER 2. UNIVERSAL SR EDM MEASUREMENT PROBLEMS AND THEIR SOLUTIONS

In Figures 2.9, 2.10, 2.11, and 2.12 are plotted, respectively: spin tune, the radial, vertical, and longitudinal components of the ISA, computed at the trajectories plotted in Figure 2.8, in two cases: (1) sextupoles are turned off, and (2) GSY sextupoles are turned on.

From the analysis of the figures, we can gather the following:

(1) in the sextupoles-off case, both $\nu_{s}$ and the direction of $\bar{n}$ are mostly (modulo the linear Taylor expansion term) fixed by the value of the particle's transverse emittance;

(2) in the sextupoles-on case, the mean levels of $\nu_{s}$ and $\bar{n}$ of different particles come together, and the betatron motion effect, related to the presence of a linear Taylor expansion term, becomes apparent.

Hence, Figures 2.10 and 2.11 are evidence that not only are the frequencies but also the directions of the beam particles' spin precession angular velocity vectors are equalized when sextupole fields are used to suppress spin decoherence. The longitudinal component of the ISA is insensitive to the sextupole fields, as evidenced by Figure 2.12 .

In Figure 2.13 we plotted the mean levels of the radial and vertical ISA components' as functions of the mean spin tune level. Based on this figure, we conclude in section 2.5 that particles having equal effective Lorentz factor values are equivalent in terms of their spin dynamics in the general (direction and magnitude of the spin precession angular velocity vector) sense. 4

\section{Analysis of the sextupole spin decoherence suppression mechanism}

From equations (1.6) and (1.8), the dependence of spin tune of the particle equilibrium energy can be expressed as:

$\nu_{s}=G \gamma_{0}+G \frac{\gamma_{0}^{2}-1}{\gamma_{0}} \cdot C_{0} \cdot f_{1}\left(\epsilon_{x}, \epsilon_{y}, Q_{x}, Q_{y}\right)+G \frac{\gamma_{0}^{2}-1}{\gamma_{0}} \cdot C_{0} \cdot f_{2}\left(\alpha_{1},\langle\Delta K / K\rangle^{2}\right)$,

${ }^{4}$ At least this seems to be true when operating in the frozen spin regime. 
CHAPTER 2. UNIVERSAL SR EDM MEASUREMENT PROBLEMS AND THEIR SOLUTIONS

where $C_{0}$ is a constant, $f_{1}$ and $f_{2}$ are defined in equation (1.8).

Since a betatron-oscillating particle does also synchrotron oscillations, the effect of sextupole fields on it is a superposition of effects. A particle injected onto the reference orbit, but having an initial energy offset, does only synchrotron oscillations. Consequently, sextupole fields affect its spin tune by only modifying the momentum compaction factor, i.e. $f_{2}$.

In view of that, we carried out a simulation in which we consecutively injected two beams of 30 particles: in the first one, the D-bunch, particles were distributed as $\delta \sim N\left(0,0.5 \cdot 10^{-6}\right)$, in the second one, the Y-bunch, as $y \sim N(0,0.5) \mathrm{mm}$. All the other phase space coordinates were set to zero at injection.

The tests were done in the ideal FS lattice in order to exclude effects associated with perturbations of non-reference (betatron) orbits. For the D-bunch, only the GSD sextupoles were turned on; for the Y-bunch GSY. The sextupole gradients were varied $\pm 5 \cdot 10^{-3}$ of the corresponding family's optimal gradient value.

Spin tracking was done for $1.2 \cdot 10^{6}$ turns, data were recorded every 800 turns.

In Figure 2.14 we plotted the longitudinal phase space portraits of both bunches. We see that the D-bunch phase portraits are practically all centered at the same point, ${ }^{5}$ and that their emittances do not change when the sextupole strength is varied.

At the same time, the Y-bunch phase portraits vary with the sextupole field strength. We observe that the the ellipse centers (i.e. the equilibrium energy levels) are most compressed at a gradient value that is not optimal (the phase portraits for the latter occupy the middle panel). This observation was what motivated us to try to inject the D-bunch in the first place. We explain this phenomenon by the superposition of the orbit length and momentum compaction factor effects.

For a more thorough analysis of the sextupole field effects on the functions $f_{1}$ and $f_{2}$ we plotted time-averaged spin tune level as a function of

\footnotetext{
${ }^{5}$ When zooming in, one can see that the ellipse centers are slightly different, but this difference is insensitive to the sextupole gradient value, and most likely is a result of finite statistics.
} 
CHAPTER 2. UNIVERSAL SR EDM MEASUREMENT PROBLEMS AND THEIR SOLUTIONS

the corresponding time-averaged energy level at different sextupole field strengths (Figure 2.15). 6 One can see from the figure that the point distribution density in the D-bunch plot does not vary as we change the gradient; the only thing that does change is the functional dependence of spin tune on the mean energy level, as is expected from the functional form of $f_{2}$ (cf. section 1.2). Hence, we concluded that the signature of the sextupole field momentum compaction effect is the change in the functional form of $\left\langle\nu_{s}\right\rangle=f(\langle\Delta K / K\rangle)$.

In the Y-bunch plot one observes two effects: both the point distribution density (i.e. the beam's longitudinal emittance) and the functional form of $\left\langle\nu_{s}\right\rangle(\langle\Delta K / K\rangle)$ change. Since the functional form change is attributed to the momentum compaction factor effect, we attribute the point density to the orbit length effect.

Conclusion: The simulation confirms statements (2.3) and (2.4).

\subsection{Machine imperfections error}

Systematic errors due to physical imperfections of the accelerator lattice, including optical element misalignments, are causative to an EDM-faking signal related to MDM spin precession [18, p. 230] Rotational magnet misalignments are particularly problematic in this respect, since they induce parasitic horizontal magnetic field components $B_{x}$ and $B_{z}$, both of which precess spin in the vertical plane; the one in which the EDM is searched for.

Y. Senichev made analytical estimates [20] of the radial component of the spin precession angular velocity. From the T-BMT equation, and the expression for the Lorentz force, its radial component can be expressed as

$$
\sigma\left[\Omega_{x}^{M D M}\right]=\frac{q}{m \gamma} \frac{G+1}{\gamma} \frac{\sigma\left[B_{x}\right]}{\sqrt{n}}
$$

\footnotetext{
${ }^{6}$ Practically, we took spin tune and ISA values corresponding to actual particle trajectories in the accelerator. So, "correspondence" here means that spin tune and kinetic energy are strictly linked to one another via a trajectory.
} 
CHAPTER 2. UNIVERSAL SR EDM MEASUREMENT PROBLEMS AND THEIR SOLUTIONS

where $n$ is the number of tilted spin-rotator elements, 7 and $\sigma\left[B_{x}\right]=$ $B_{y} \sigma[\delta h] / L$, with the misalignment error standard deviation $\sigma[\delta h]$. Assuing $\sigma[\delta h]=100 \mu \mathrm{m}$, and spin-rotator length $L=1 \mathrm{~m}, \sigma\left[\Omega_{x}^{M D M}\right] \approx 100$ $\mathrm{rad} / \mathrm{sec} .[20$ ]

We analyzed the particle spin dynamics in the imperfect FS and QFS lattices using COSY Infinity. [31] Our simulation results tend to confirm the above estimates.

Imperfection field implementation When implementing machine imperfections we followed recommendations given in [18, p. 235]. A small perturbation of the magnetic field acts like a proportional rotation of the spin vector. For this reason we implemented the $\mathrm{E}+\mathrm{B}$ element tilt as a product between the element's spin transfer matrix and the corresponding rotation matrix, a "spin kick." Such an implementation guarantees the preservation of the closed orbit. This orbit preservation is physically grounded in the fact that when a spin-rotator is tilted, there emerges a compensating electric field keeping the Lorentz force constant.

According to equation (1.1b), a change in the MDM precession angular velocity associated with the presence of a parasitic magnetic field $\left(B_{x}, 0, B_{z}\right)$ is

$$
\Delta \Omega_{M D M}=\frac{q}{m} G \cdot\left(B_{x}, 0, B_{z}\right),
$$

hence the spin kick angle

$$
\Theta_{\text {kick }}=t_{0} \Delta \Omega_{M D M},
$$

where $t_{0}=L / v_{0}$ is the reference particle's time of flight through the element.

\section{Tilt distribution dependence}

This series of simulations was carried out in order to prove (or reject) the validity of two theses concerning the machine imperfection systematic

\footnotetext{
${ }^{7}$ The estimates were made for the FS lattice described in section 1.3
} 
CHAPTER 2. UNIVERSAL SR EDM MEASUREMENT PROBLEMS AND THEIR SOLUTIONS

error: (1) the induced MDM spin precession angular velocity component is independent of the particular element tilt distribution, and depends only on the mean tilt angle; and (2) this dependence is linear.

The simulation was set up as follows: in the FS lattice described in section $1.3 \mathrm{E}+\mathrm{B}$ elements were randomly tilted about the optic axis by angles $\Theta_{\text {tilt }}$. After building the third-order spin and orbital transfer maps, we computed the Taylor expansions of the spin tune and spin precession axis (SPA). The zero-order terms of the Taylor expansions represent the spin tune and SPA of the reference particle.

The reference particle spin precession angular velocity is calculated according to equation: [15, p. 4]

$$
\boldsymbol{\Omega}=2 \pi / \tau_{0} \cdot \nu_{s} \cdot \bar{n}
$$

where $\tau_{0}=f_{\text {rev }}^{-1}=10^{-6}$ seconds is the particle's time of flight through the full lattice.

The simulation was carried out 11 times; each time the spin-rotator tilt angles were picked from a normal distribution $N\left(\mu_{0} \cdot(i-5), \sigma_{0}\right)$, where $\mu_{0}=10 \cdot \sigma_{0}=10^{-4} \mathrm{rad}, i \in\{0, \ldots, 10\}$. The simulation results are plotted in Figure 2.16 .

One can observe from the figure that a tilt distribution at which the mean tilt angle is equal to $10^{-4}$ radians, the beam polarization vector precesses in the vertical plane at the rate of $500 \mathrm{rad} / \mathrm{sec}$. This agrees with the estimates mentioned above (section 2.3), because in them a tilt error standard deviation of $10^{-4} \mathrm{rad}$ is assumed at 100 tilted elements. In that case, the mean tilt angle standard deviation is $10^{-5}$, and hence MDM precession occurs at a rate up to $50 \mathrm{rad} / \mathrm{sec}$ with a probability $67 \%$, and up to $100 \mathrm{rad} / \mathrm{sec}$ with a probability $95 \%$.

Figure 2.17 shows the results of a simulation in which six randomlypicked $\mathrm{E}+\mathrm{B}$ elements were pair-wise tilted by opposite angles, while one element was tilted by an angle $\mu_{i}=(i-5) \cdot 10^{-6} \mathrm{rad}, i \in\{0, \ldots, 10\}$.

Both simulations were done at the strict FS energy $270.0092 \mathrm{MeV} .8$

\footnotetext{
${ }^{8}$ At this energy, in the ideal lattice, $\nu_{s}$ and $\bar{n}$ are undefined in the beam rest frame used in COSY Infinity. This corresponds to the situation when spin does not precess in any plane
} 
CHAPTER 2. UNIVERSAL SR EDM MEASUREMENT PROBLEMS AND THEIR SOLUTIONS

One can see that the compensated elements do not contribute to the spin precession.

\section{Comparison of the CW vs CCW beams' spin precession angular velocities}

In Figure 2.18 we plotted the relative difference between the CW and CCW beams' radial SPA/angular velocity components in the case of both the normally-distributed and mutually-compensated tilt cases.

For the raidal SPA component the relative difference was computed as

$$
\delta \bar{n}_{x}=\frac{\bar{n}_{x}^{C W}\left(\left\langle\Theta_{t i l t}\right\rangle\right)-\bar{n}_{x}^{C C W}\left(\left\langle\Theta_{t i l t}\right\rangle\right)}{\bar{n}_{x}^{C W}\left(\left\langle\Theta_{t i l t}\right\rangle\right)} ;
$$

for the angular velocity:

$$
\delta \Omega_{x}=\frac{\Omega_{x}^{C W}\left(\left\langle\Theta_{t i l t}\right\rangle\right)-\Omega_{x}^{C C W}\left(\left\langle\Theta_{t i l t}\right\rangle\right)}{\Omega_{x}^{C W}\left(\left\langle\Theta_{t i l t}\right\rangle\right)} .
$$

In the figures, one can observe that in either case both beams' SPA is oriented the same way; there is some difference between the beams' spin tunes, but it stays below the percent level. The spin tune difference grows bigger as the spin wheel roll rate (proportional to the mean tilt angle) gets slower. The spin tune difference may indicate that the lattice is asymmetric, with respect to the spin dynamics, relative to the beam circulation direction (i.e. time reversal). It may be explained by a difference between the CW and CCW beams' closed orbits.

\subsection{Guide field flipping}

Two aspects of the problem need to be paid attention to:

(either horizontal or vertical), which corresponds to the realization of the 3D FS condition in an ideal lattice. 
CHAPTER 2. UNIVERSAL SR EDM MEASUREMENT PROBLEMS AND THEIR SOLUTIONS

(1) What needs to be kept constant from one measurement cycle to the next;

(2) How it can be observed.

The goal of flipping the direction of the guide field is to accurately reproduce the radial component of the MDM spin precession frequency induced by machine imperfection fields. This point should not be overlooked: a mere reproduction of the magnetic field strength would not suffice, since the injection point of the beam's centroid, and hence its orbit length - and, via equations (1.10) and (1.6), spin tune, - is subject to variation. (Apart from that, the accelerating structure might not be symmetrical, in terms of spin dynamics, with regard to reversal of the beam circulation direction.)

What needs to be reproduced, therefore, is not the field strength, but the effective Lorentz factor of the centroid.

Regarding the second question, we mentioned earlier that the Koop Wheel roll rate is controlled through measurement of the horizontal plane spin precession frequency. This plane was chosen because the EDM angular velocity vector points (mainly) in the radial direction; its vertical component is due to machine imperfection fields, and is small compared to the measured EDM effect. Therefore, in first approximation, when we manipulate the vertical component of the combined spin precession angular velocity, we manipulate the vertical component of the MDM angular velocity vector.

The effective Lorentz factor calibraion procedure consists in the following.

\section{Calibration algorithm}

Let $\mathcal{T}$ denote the set of all trajectories that a particle might follow in the accelerator. $\mathcal{T}=\mathcal{S} \cup \mathcal{F}$, where $\mathcal{S}$ is the set of all stable trajectories, $\mathcal{F}$ are all trajectories such that if a particle gets on one, it will be lost from the bunch.

Calibration is done in two phases: 


\section{CHAPTER 2. UNIVERSAL SR EDM MEASUREMENT PROBLEMS} AND THEIR SOLUTIONS

(1) In the first phase, the guide field value is set so that the beam particles are injected onto trajectories $t \in \mathcal{S}$.

(2) In the second phase, it is fine-tuned further, so as to fulfill the FS condition in the horizontal plane. By doing this, we physically move the beam trajectories into the subset $\left.\mathcal{S}\right|_{\Omega_{y}=0} \subset \mathcal{S}$ of trajectories for which $\omega_{y}=0$.

Spin tune (and hence precession frequency) is an injective function of the effective Lorentz-factor $\gamma_{\text {eff }}$, which means $\omega_{y}\left(\gamma_{\text {eff }}^{1}\right)=\omega_{y}\left(\gamma_{\text {eff }}^{2}\right) \rightarrow$ $\gamma_{\text {eff }}^{1}=\gamma_{\text {eff }}^{2}$. The trajectory space $\mathcal{T}$ is partitioned into equivalence classes according to the value of $\gamma_{e f f}$ : trajectories characterized by the same $\gamma_{e f f}$ are equivalent in terms of their spin dynamics (possess the same spin tune and invariant spin axis direction), and hence belong to the same equivalence class. Since $\omega_{y}\left(\gamma_{e f f}\right)$ is injective, there exists a unique $\gamma_{e f f}^{0}$ at which $\omega_{y}\left(\gamma_{\text {eff }}^{0}\right)=0$ :

$$
\left[\omega_{y}=0\right]=\left.\left[\gamma_{\text {eff }}^{0}\right] \equiv \mathcal{S}\right|_{\Omega_{y}=0} .
$$

If the lattice didn't use sextupole fields for the suppression of decoherence, $\left.\mathcal{S}\right|_{\Omega_{y}=0}$ would be a singleton set. We have shown in 2.2 that if sextupoles are utilized, then $\exists \mathcal{D} \subset \mathcal{S}$ such that $\forall t_{1}, t_{2} \in \mathcal{D}: \nu_{s}\left(t_{1}\right)=\nu_{s}\left(t_{2}\right)$, $\bar{n}\left(t_{1}\right)=\bar{n}\left(t_{2}\right)$. By adjusting the guide field strength we equate $\mathcal{D}=\left.\mathcal{S}\right|_{\Omega_{y}=0}$, and hence $\left.\mathcal{S}\right|_{\Omega_{y}=0}$ contains multiple trajectories. ${ }^{9}$

Therefore, once we ensured that the beam polarization does not precess in the horizontal plane, all of the beam particles have $\gamma_{e f f}^{0}$, equal for the CW and CCW beams.

In order to confirm that the proposed calibration procedure works, we need to show that:

(1) $\left.\mathcal{S}\right|_{\Omega_{y}=0} ^{C W}=\left.\mathcal{S}\right|_{\Omega_{y}=0} ^{C C W}$, that is $\Omega_{y}=0$ for the same set of trajectories (equivalently, the same $\gamma_{e f f}$ ) in the $\mathrm{CW}$ and CCW cases.

\footnotetext{
${ }^{9}$ Strictly speaking, even if sextupoles are used there remains some negligible dependence of spin tune on the particle orbit length (linear decoherence effects, cf. 2.2). Because of that, the equalities for $\nu_{s}$ and $\bar{n}$ are approximate, and the set $\left.\mathcal{S}\right|_{\Omega_{y}=0}$ should be viewed as fuzzy: we will consider trajectories for which $\left|\omega_{y}\right|<\delta$ for some small $\delta$ as belonging to $\left[\omega_{y}=0\right]$.
} 
CHAPTER 2. UNIVERSAL SR EDM MEASUREMENT PROBLEMS AND THEIR SOLUTIONS

(2) $\forall t_{1},\left.t_{2} \in \mathcal{S}\right|_{\Omega_{y}=0} ^{C C W}: \nu_{s}\left(t_{1}\right)=\nu_{s}\left(t_{2}\right), \bar{n}\left(t_{1}\right)=\bar{n}\left(t_{2}\right)$, i.e., the same sextupole fields reduce decohrerence in the CW and CCW beams.

Practically, we do this by:

(1) computing the dependencies $\nu_{s}(z), z \in\{x, y, \delta\}$ for the CW and CCW beams;

(2) computing the discrepancy $\epsilon(z)=\nu_{s}^{C W}(z)-\nu_{s}^{C C W}(z)$.

If the discrepancy is small in a wide range of $z$, then

(1) sextupole decoherence suppression works for both beams without gradient value change;

(2) spin tune (respectively $\gamma_{e f f}$ ) is equal for both beams, and hence their Spin Wheels roll at the same rate.

The $\bar{n}^{C W}, \bar{n}^{C C W}$ tilt angles relative to the closed orbit plane are determined by the accuracy of setting $\Omega_{y}=0$.

\section{Simulation}

In the simulation, we use an imperfect FS lattice [28], in which the $\mathrm{E}+\mathrm{B}$ spin rotator elements are tilted about the optic axis by angles

$$
\alpha \sim N\left(0,5 \cdot 10^{-4}\right) \mathrm{rad}
$$

Spin decoherence is being suppressed. The simulation is repeated three times; each time only one sextupole family is turned on. Each family's sextupole gradieent is optimized according to the procedure described in section 2.2 .

The beam kinetic energy is $270.00 \mathrm{MeV}$. We compute third-order Taylor expansions of the spin and orbital transfer maps.

The main body of the simulation consists in the following: using the TSS [32, p. 41] procedure of COSY Infinity we compute the $\nu_{s}$ and $\bar{n}$ thirdorder Taylor expansions for the lattice traversed in the forward direction. 
CHAPTER 2. UNIVERSAL SR EDM MEASUREMENT PROBLEMS AND THEIR SOLUTIONS

Then, using the combinations of procedures MR and SMR [18, p. 233], we reverse the lattice's orbital and spin transfer maps, and compute $\nu_{s}$ and $\bar{n}$ for the reversed lattice (as it is seen by the counter-circulating beam).

\section{Results}

The test results are shown in Figures 2.19, 2.20, and 2.21. Specifically, in Figures 2.19a, 2.20a, and 2.21a we plotted CW and CCW beams' $\nu_{s}$ and $\bar{n}_{y}$ as functions of the particle's transverse $(x, y)$ and energy $(\delta)$ phase space coordinates, respectively. One can see that the $\nu_{s}^{C W}$ and $\nu_{s}^{C C W}$ dependencies (as well as $\bar{n}_{y}^{C W}$ and $\bar{n}_{y}^{C C W}$ ) differ, but at the same time the beams' $\Delta \Omega_{y}$ discrepancy does not exceed $\pm 3 \cdot 10^{-6} \mathrm{rad} / \mathrm{sec}$; the spin tune discrepancy is below $10^{-13}$, that of the transverse components of $\bar{n}$ the $10^{-8}$ level. Figures 2.19b, 2.20b, and 2.21b show the difference between the CW \& CCW beams' spin precession angular velociy vectors' radial components as a function of their vertical component difference. One can observe, that when the difference $\Delta \Omega_{y}<10^{-7} \mathrm{rad} / \mathrm{sec}$ (this is the statistical precision of a frequency estimate achievable in one cycle), the difference $\Delta \Omega_{x}<10^{-8} \mathrm{rad} / \mathrm{sec}$ (i.e., an order of magnitude less than the statistical precision). This confirmes that the equalization of the vertical plane MDM precession frequencies of counter-circulating beams by means of equalizing their horizontal plane precession frequencies is a viable technique.

\subsection{Spin tune equivalence of trajectories of equal effective Lorentz factor}

In the context of the spin wheel roll direction change procedure, it is important to consider the question of the $\mathrm{CW}$ and $\mathrm{CCW}$ beams' equivalence in trems of their spin dynamics.

Our analysis starts from Statement 1: particles having an equal effective Lorentz factor value have equal spin tunes, i.e. are equivalent in their spin dynamics. This is a consequence of equation (1.6).

In the next sections we will consider two formulations of Statement 1: 
CHAPTER 2. UNIVERSAL SR EDM MEASUREMENT PROBLEMS AND THEIR SOLUTIONS

A. when interpreting the effective L-factor as the expectation value of the particle energy;

B. the multivariate function $\nu_{s}(x, a, y, b, \ell, \delta)$ is agnostic to the paricle's trajectory in the transverse phase planes $(x, a)$, and $(y, b)$, that is, it can be reduced to a multivariable function $\nu_{s}\left(\gamma_{e f f}\right)$.

\section{Formulation A}

In this section we will consider Statement 1, interpreting the effective Lorentz factor as the expectation value of a particle's Lorentz factor.

In order to test this formulation we carried our the following simulation: we injected three 10-particle bunches (X, Y, and D) into the ideal FS lattice. The orbital and spin transfer maps were computed up to the third-order Taylor expansion; the particle injection energy was $270 \mathrm{MeV}$. The X-bunch particles were uniformly distributed along the radial axis in the range $\pm 1 \mathrm{~mm}$; those of the $\mathrm{Y}$-bunch, along the vertical axis in the range $\pm 1.318 \mathrm{~mm} ;{ }^{10}$ the $\mathrm{D}$-bunch particles were distributed by $\Delta K / K_{0}$ in the range $\pm 10^{-4}$. Then, spin tracking was done for 12,000 turns, with data recorded every 80 turns.

The recorded data were:

(i) the particle phase space coordinate $\boldsymbol{z}=\left(x, x^{\prime}, y, y^{\prime}, \ell, \delta\right)$, where $\ell=-\left(t-t_{0}\right) v_{0} \frac{\gamma_{0}}{1+\gamma_{0}}$ is its longitudinal phase offset, $\delta=\Delta K / K$ is the energy offset;

(ii) spin tune $\nu_{s}(\boldsymbol{z})$.

Based on these data we computed the particles' time-average spin tune $\left\langle\nu_{s}\right\rangle$, energy offset $\langle\Delta K / K\rangle$, and longitudinal and transverse emittances.

Simulation results are presented in Figure 2.22. The top panel is a plot of $\left\langle\nu_{s}\right\rangle$ as a function of $\langle\Delta K / K\rangle$ for the betatron-oscillating bunches

\footnotetext{
${ }^{10}$ This range was chosen in order to equalize the transverse emittances of the particles. The initial coordinate offset determines the betatron oscillation amplitude $A$, which is related to the beta function $\beta$ and transverse emittance $\epsilon$ as in $A=\sqrt{\epsilon \beta}$.
} 
CHAPTER 2. UNIVERSAL SR EDM MEASUREMENT PROBLEMS AND THEIR SOLUTIONS

when the sextupoles are turned off. One can see from the figure that, at the same mean energy level, the horizontal plane betatron oscillating particles have a different spin tune from that of the vertical plane betatron oscillating particles. This means, as far as we can tell, that Statement 1 in formulation A is disproved.

We hypothesized that the difference in the plotted lines' slopes is related to the spatial dependence of the momentum compaction factor.

This hypothesis is based on our analysis of the sextupole field suppression effects' signatures, described in detail in section 2.2. In order to test this hypothesis we repeated the experiment at different values of the GSX sextupole gradient, taken from the range $\pm 5 \cdot 10^{-3}$. The simulation results are shown in Figure 2.22. The same dependence is plotted as previously, but only for the X-bunch. As one can see, when the gradient is varied the slope varies with it. The same behavior as was observed in section 2.2 .

In order to check the hypothesis about the spatial dependence of the momentum compaction factor we computed the dependencies of the mean energy levels of the $\mathrm{X}$ - and Y-bunch particles on their betatron tunenormalized transverse emittances (Fiugre 2.23). According to equation (1.9), the orbit lengthening of particles with equal Q-normalized transverse emittances must be equal. The equilibrium energy level shift of a particle is proportional to its orbit lengthening via the momentum compaction factor; hence the slope difference seen in Figure 2.23 is evidence that the momentum compaction factors experienced by the $\mathrm{X}$-, and Y-bunches are different.

The observed longitudinal dependence of the momentum compaction factor is further confirmed by equation (15) of reference [27], in which we find:

$$
\alpha_{0}=\left\langle\frac{D_{0}}{\rho}\right\rangle, \quad \alpha_{1}=\left\langle\frac{D_{1}}{\rho}\right\rangle+\frac{1}{2}\left\langle D_{0}^{\prime 2}\right\rangle,
$$

where $D(s)=D_{0}(s)+D_{1}(s) \cdot \delta$ is the dispersion function, $\rho$ the radius of the clsed orbit. In first approximation, dispersion exists only in the horizontal plane and is zero in the vertical plane, meaning that the spatial dependence of the dispersion function reflects on the spatial dependence of the momentum compaction factor. 
CHAPTER 2. UNIVERSAL SR EDM MEASUREMENT PROBLEMS AND THEIR SOLUTIONS

For comparison, the same tests were carried out with linear Taylor expansions of the spin and orbital transfer maps. The results are shown in Figures 2.24b, and 2.24a. As one can see in Figure 2.24a, all particles doing betatron oscillations in the vertical plane share the same value of the mean energy level, which is an indication that they share the same closed orbit, which in turn means there's no dispersion in the vertical plane. In this case, from Figure $2.24 \mathrm{~b}$ follows that their spin tunes are equal.

In Figure 2.25 are plotted the particle longitudinal emittance as a function of its Q-normalized transverse emittance. As one can see, the transverse emittances induce the longitudianl emittances at different rates, depending on the betatron oscillation plane. In the linear case, vertical plane betatron oscillations do not induce synchrotron oscillations at all.

Conclusion: formulation A of Statement 1 is false.

\section{Formulation B}

Using COSY Infinity we compute the Taylor expansion of spin tune $\nu_{s}(\boldsymbol{z})$, where

$$
\begin{aligned}
\boldsymbol{z} & =(x, a, y, b, \ell, \delta), \\
\ell & =-\left(t-t_{0}\right) v_{0} \frac{\gamma-1}{\gamma}, \\
\delta & =\frac{\Delta K}{K} .
\end{aligned}
$$

In the present section we will test formulation B of Statement 1: the multivariate function $\nu_{s}(\boldsymbol{z})$ can be expressed as a function of a single scalar parameter: $\nu_{s}\left(\gamma_{e f f}\right)$. We will not assume any formal expression of $\gamma_{e f f}$.

If formulation $\mathrm{B}$ is correct, there exists a coordinate system (with one axis being $\nu_{s}$ ), in which horizontal plane betatron oscillating particles are indistinguishable, in terms of spin tune, from vertical plane betatron oscillating particles. This coordinate system, hence, must not include coordinates from the transverse phase space planes $(x, a)$, and $(y, b)$. 
CHAPTER 2. UNIVERSAL SR EDM MEASUREMENT PROBLEMS AND THEIR SOLUTIONS

Therefore, we will look at the space $\mathcal{P}=\left(\ell, \delta, \nu_{s}\right)$. If formulation $\mathrm{B}$ is correct, differences between particles' transverse phase plane trajectories must not reflect on their trajetctories in $\mathcal{P}$.

We used the same data in this analysis as in the previous section.

In Figure 2.26a $\nu_{s}(\boldsymbol{z})$ is plotted as a function of $(\ell, \delta)$ when $\boldsymbol{z}$ is the real trajectory the particle takes in the storage ring. We observe:

(1) the same stratification of the mean spin tune levels as in figures in section 2.2 ;

(2) the stratification is more pronounced for the X-bunch (blue dots), than for the Y-bunch (red dots).

The latter can be explained by the greater magnitude of the dispersion function in the horizontal plane. Note that at equal values of the Q-normalized transverse emittance ${ }^{11}$ (i.e. at equal orbit lengthenings, if equation (1.9) is to be believed), horizontal plane betatron oscillating particles have a greater longitudinal emittance than those oscillating in the vertical plane.

Due to this fact, we decided to plot the same dependence, but to pick particles based on the equality of their longitudinal, instead of transverse, emittances. In Figure 2.26b we observe that particles having similar magnitudes of their longitudinal emittance have also silimar mean spin tune levels.

Conclusion: formulation B is confirmed by simulation; the effective Lorentz factor reflects the magnitude of the particle's longitudinal emittance.

In view of Figure 2.13, one can also conclude that particles with equal effective L-factor values are spin dynamics-equivalent in the general sense, by which we mean that they have not only equal values of spin tune, but the same orientation of the invariant spin axis. ${ }^{12}$

\footnotetext{
${ }^{11} \mathrm{Q}$-normalized is $\epsilon_{\alpha} \cdot Q_{\alpha}$, where $\alpha \in\{x, y\}$.

${ }^{12}$ At any rate, this seems to be true in the FS regime of operation.
} 
CHAPTER 2. UNIVERSAL SR EDM MEASUREMENT PROBLEMS AND THEIR SOLUTIONS

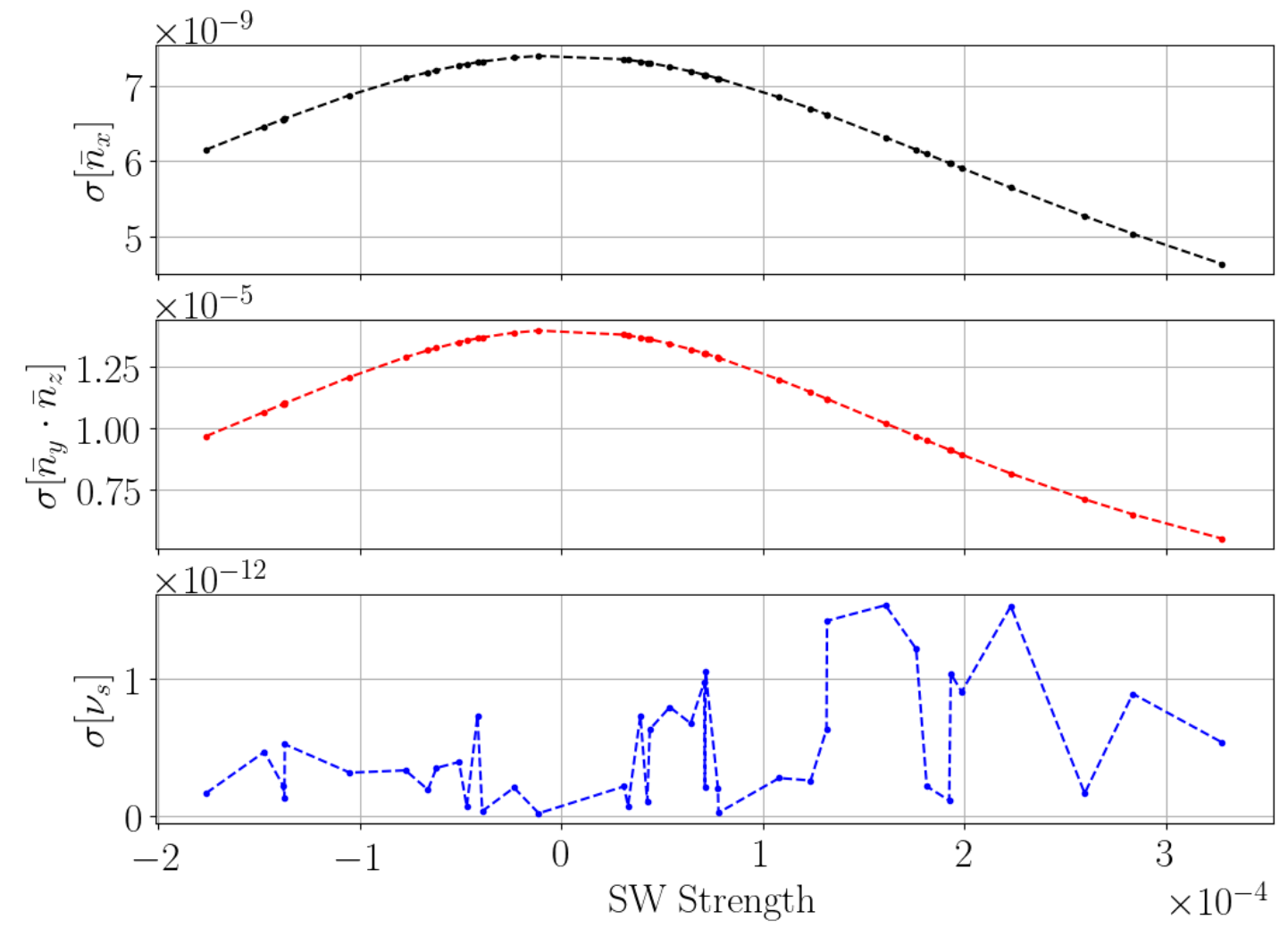

(a) $\bar{n}$ component.
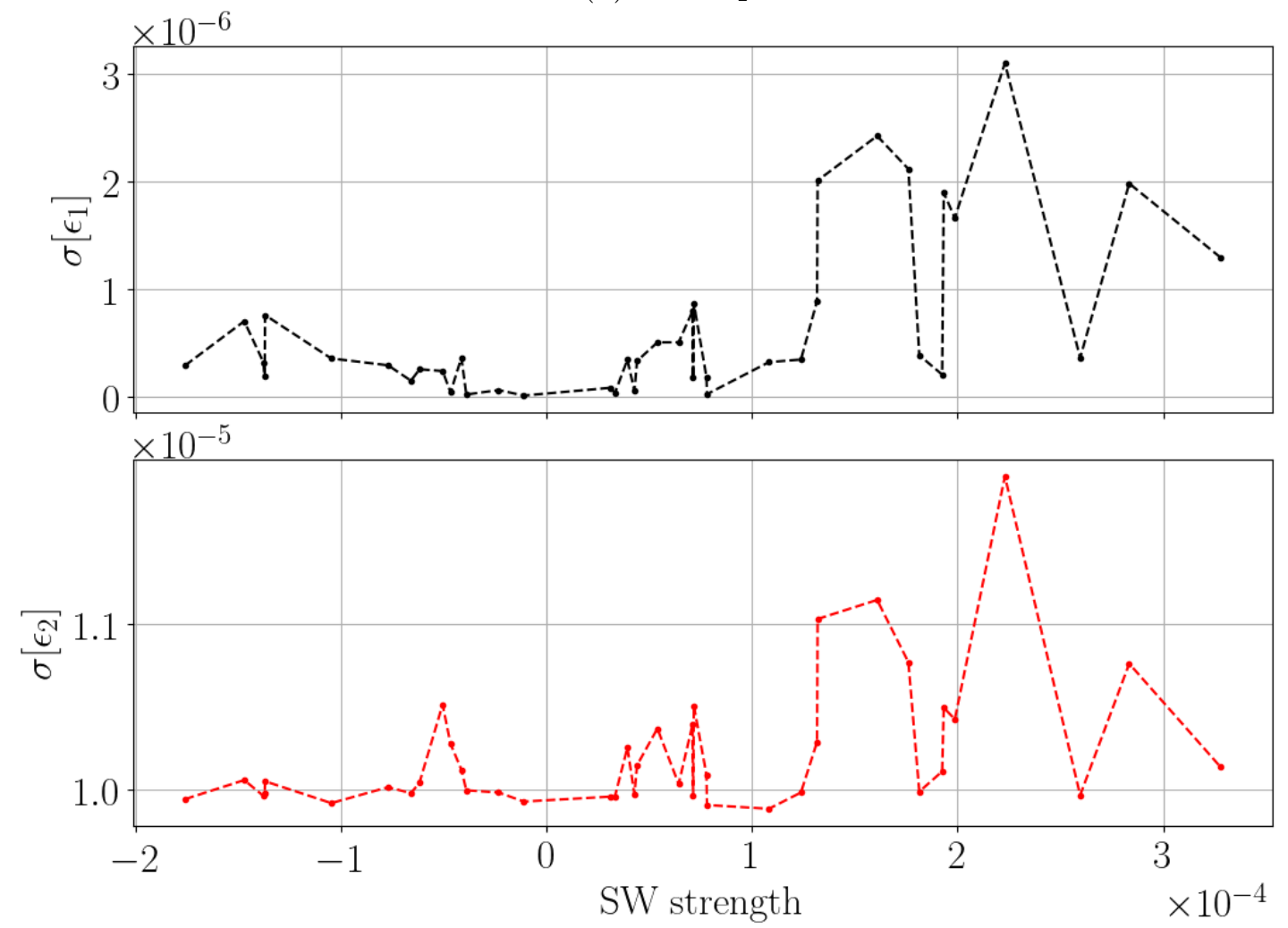

(b) Comparator residuals. Top panel: $\epsilon_{1}$ residual; bottom panel: $\epsilon_{2}$ residual

Figure 2.2: Standard deviation vs spin wheel roll rate. 
CHAPTER 2. UNIVERSAL SR EDM MEASUREMENT PROBLEMS AND THEIR SOLUTIONS

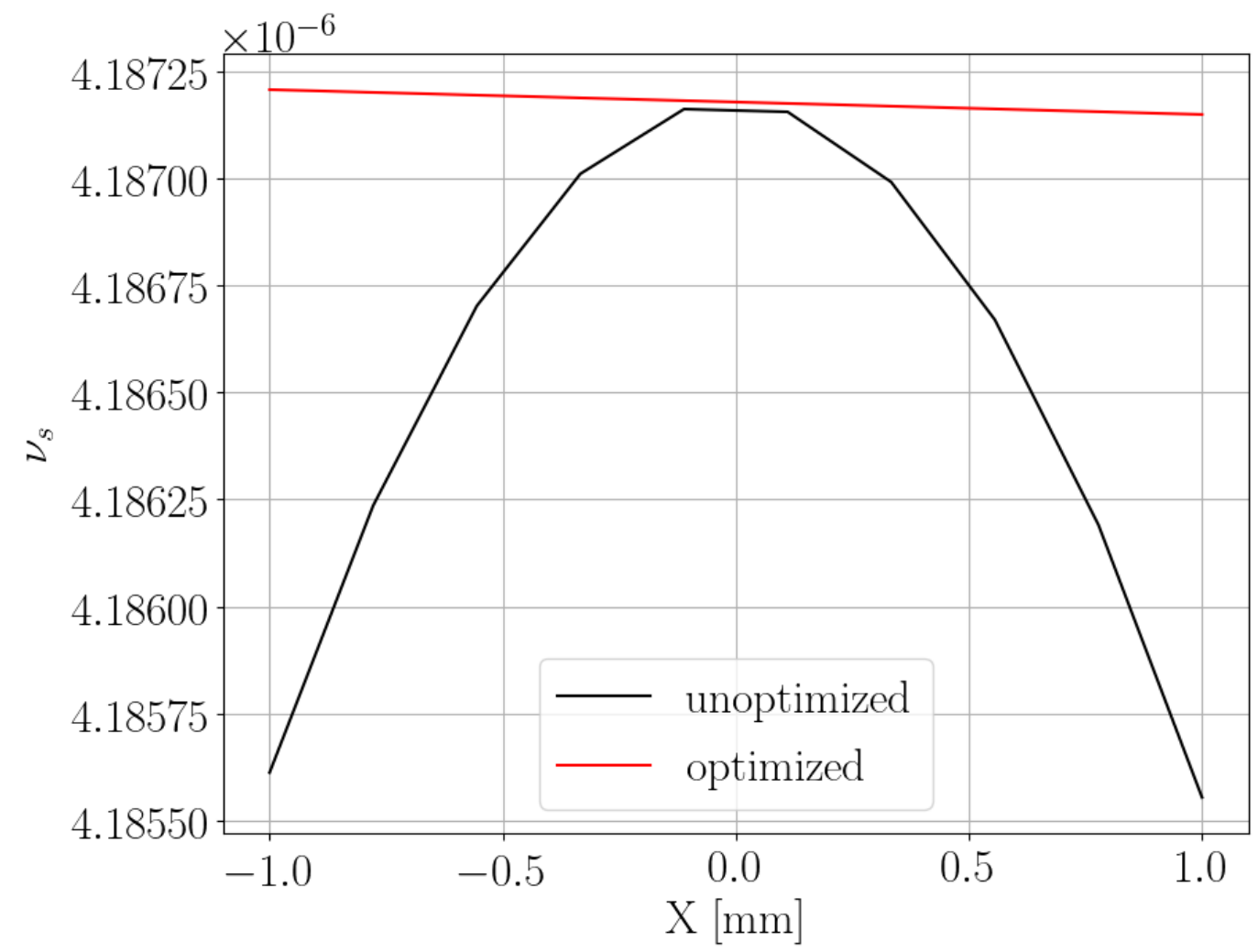

(a) Radial offset

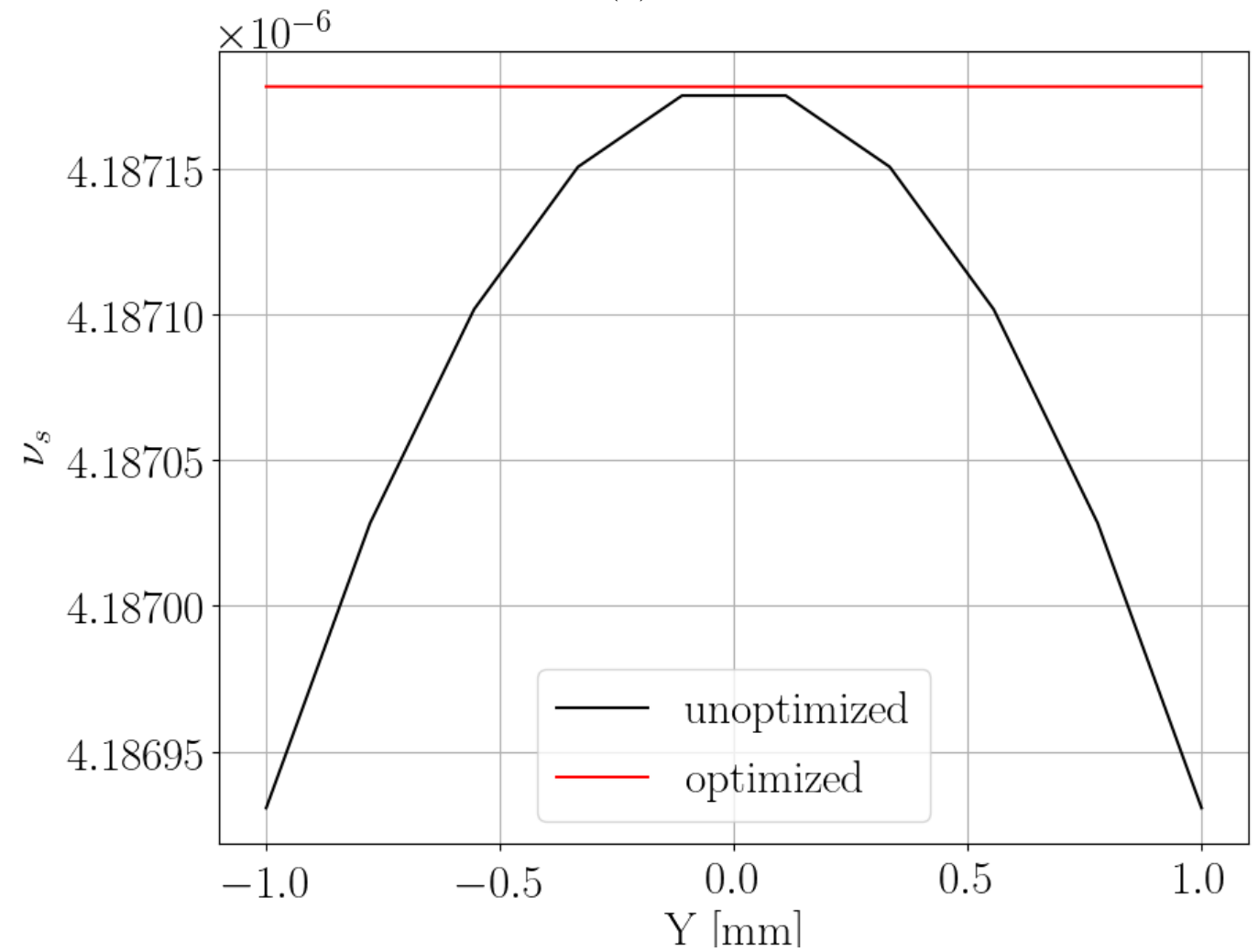

(b) Vertical offset 
CHAPTER 2. UNIVERSAL SR EDM MEASUREMENT PROBLEMS AND THEIR SOLUTIONS

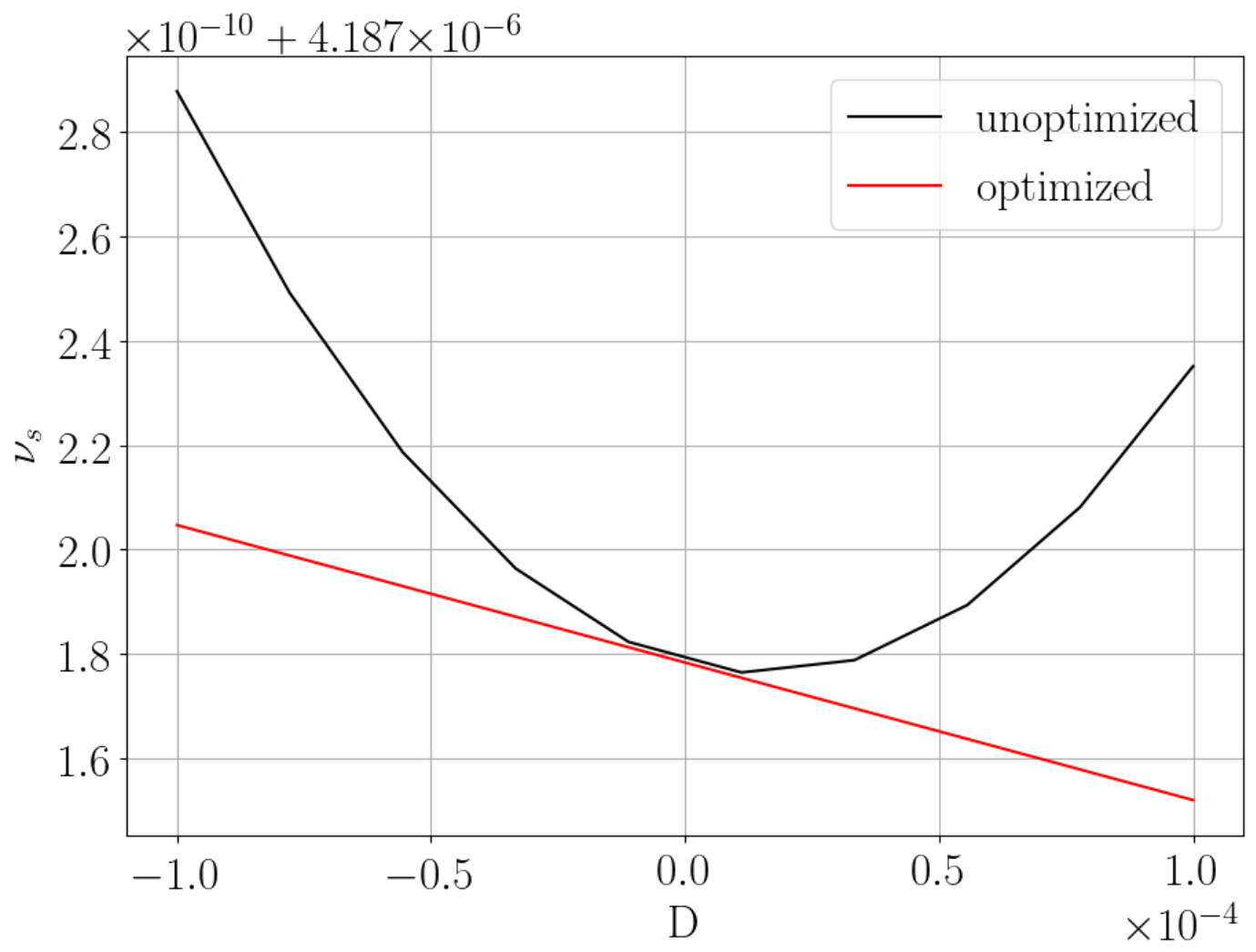

(c) Energy offset

Figure 2.3: The dependence of a particle spin tune on its initial offset from the reference particle. 
CHAPTER 2. UNIVERSAL SR EDM MEASUREMENT PROBLEMS AND THEIR SOLUTIONS

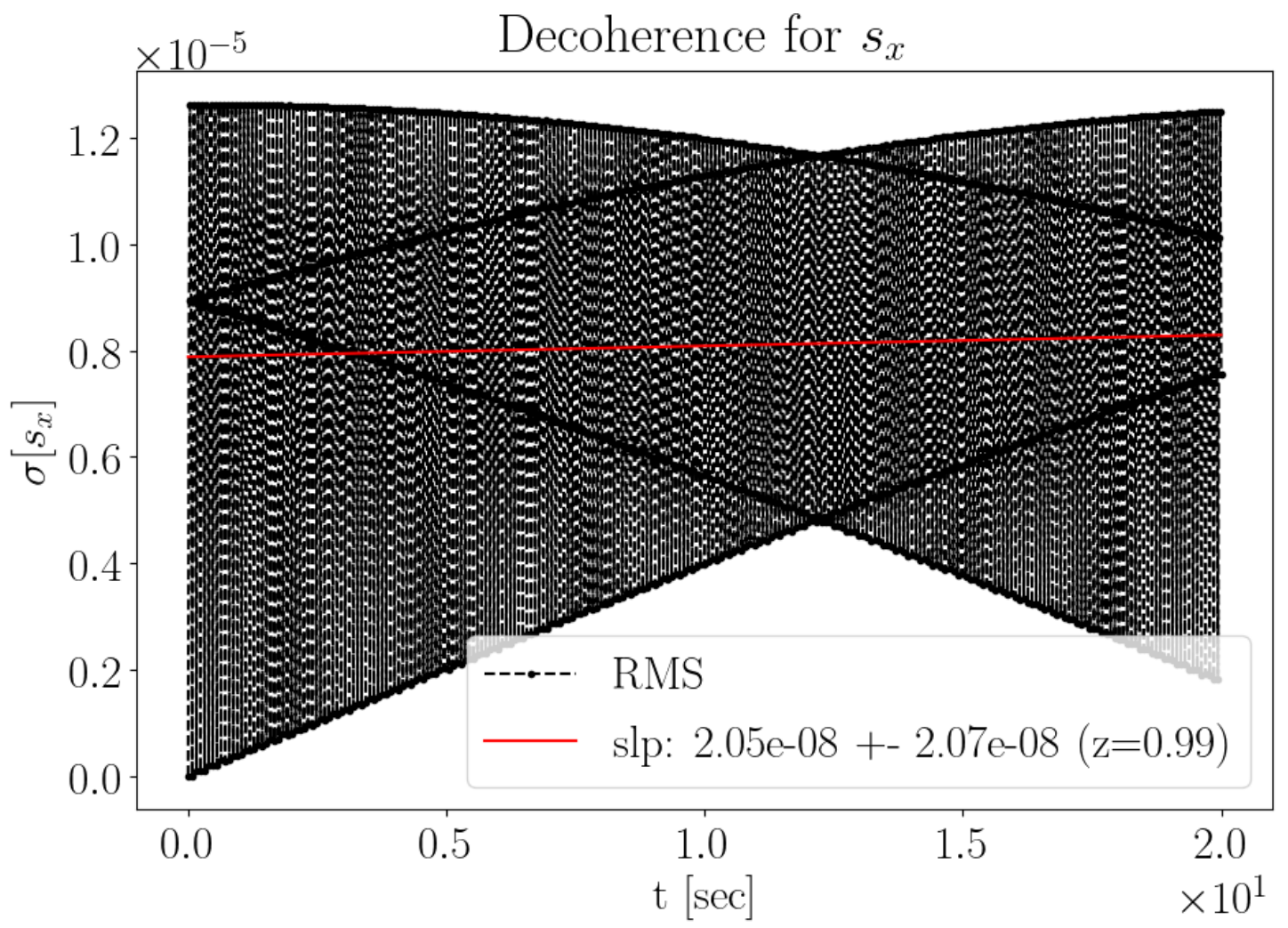

(a) Sextupoles off

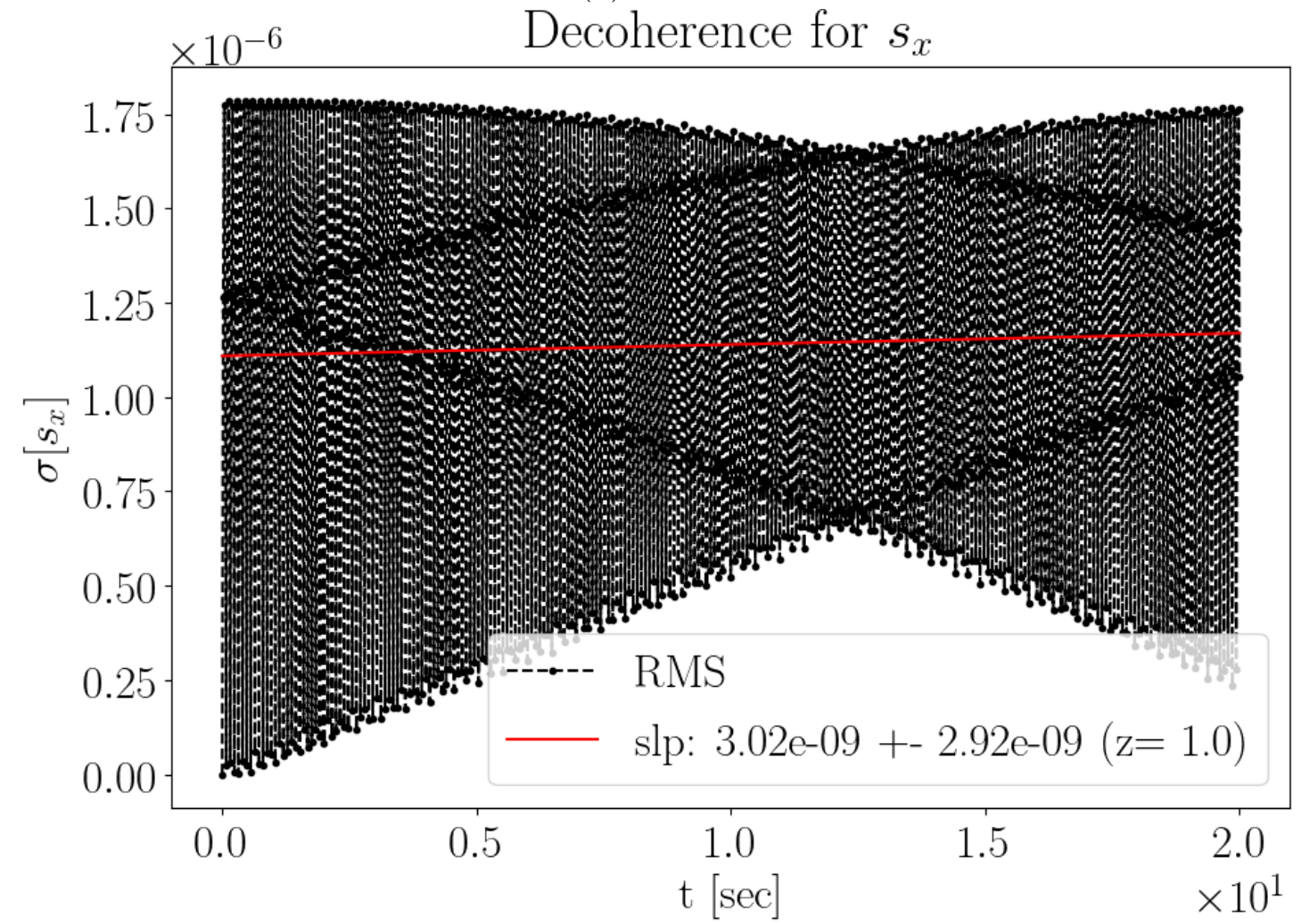

(b) Sextupoles on

Figure 2.4: Standard deviation of the radial spin vector component distribution in a bunch. 
CHAPTER 2. UNIVERSAL SR EDM MEASUREMENT PROBLEMS AND THEIR SOLUTIONS

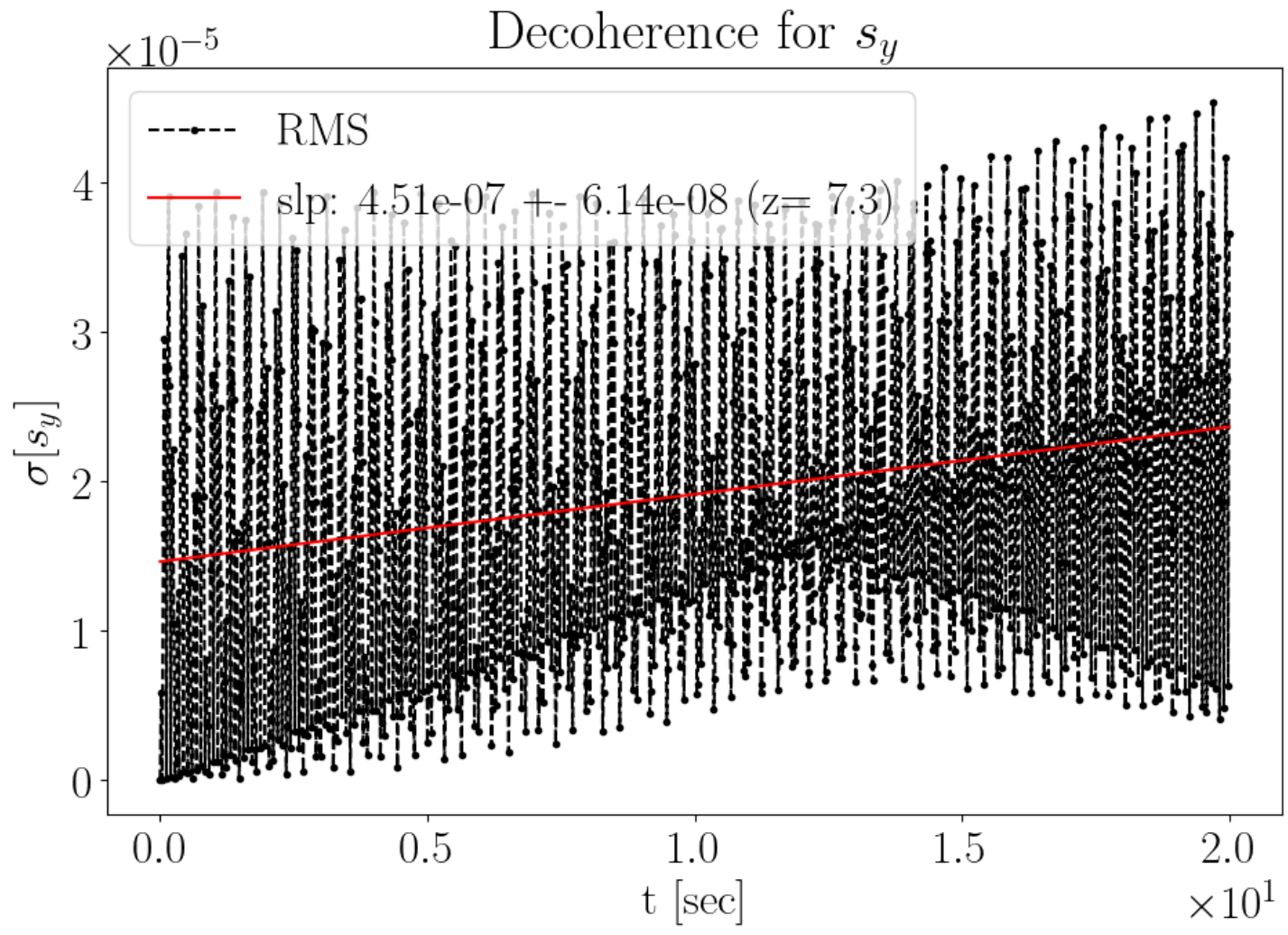

(a) Sextupoles off

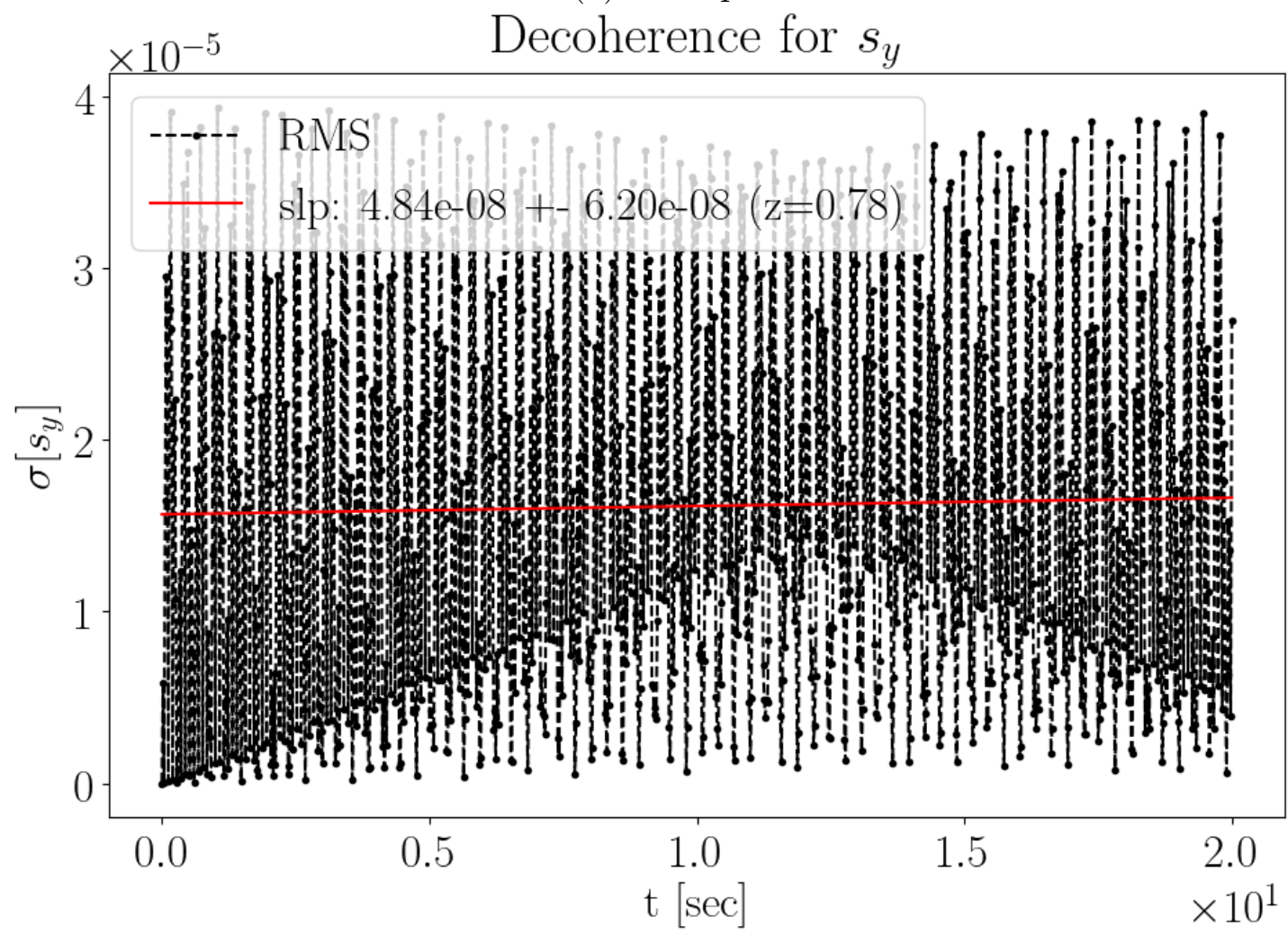

(b) Sextupoles on

Figure 2.5: Standard deviation of the radial spin vector component distribution in a bunch. 
CHAPTER 2. UNIVERSAL SR EDM MEASUREMENT PROBLEMS AND THEIR SOLUTIONS

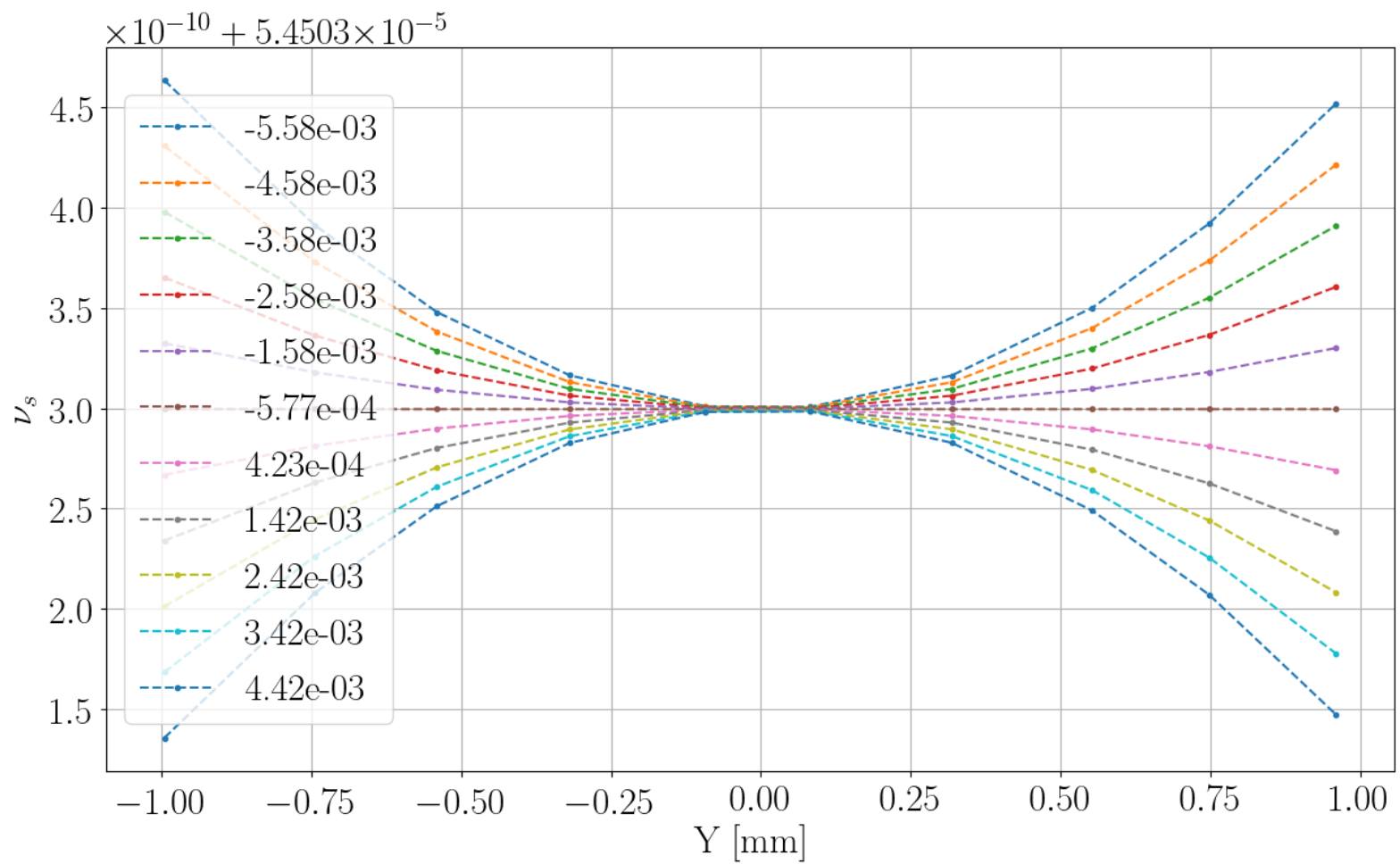

(a) Full range.

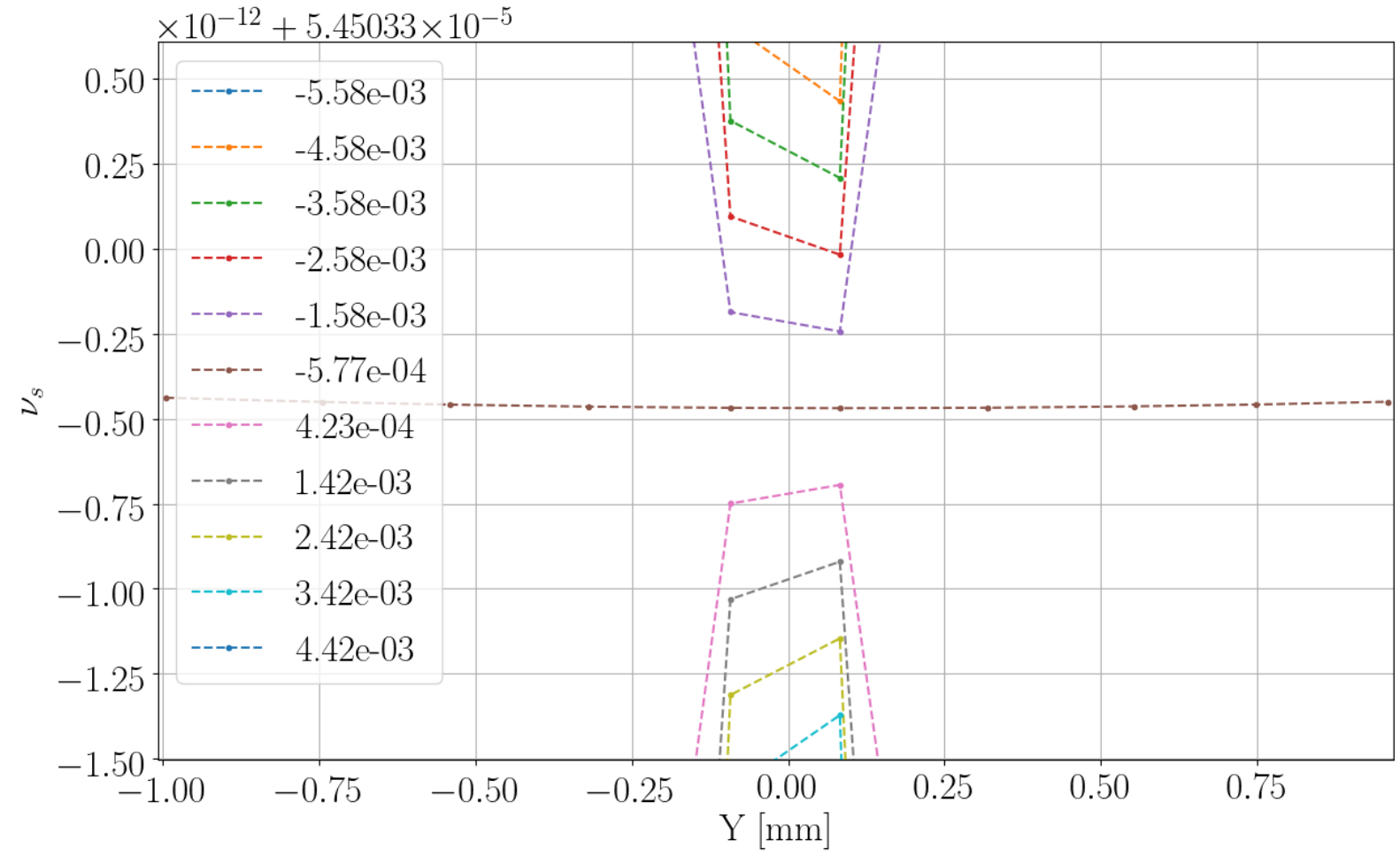

(b) Detalization.

Figure 2.6: Spin tune $\nu_{s}$ as a function of the particle's vertical offset from the closed orbit. Color marks different $G_{Y}$ values. 
CHAPTER 2. UNIVERSAL SR EDM MEASUREMENT PROBLEMS AND THEIR SOLUTIONS

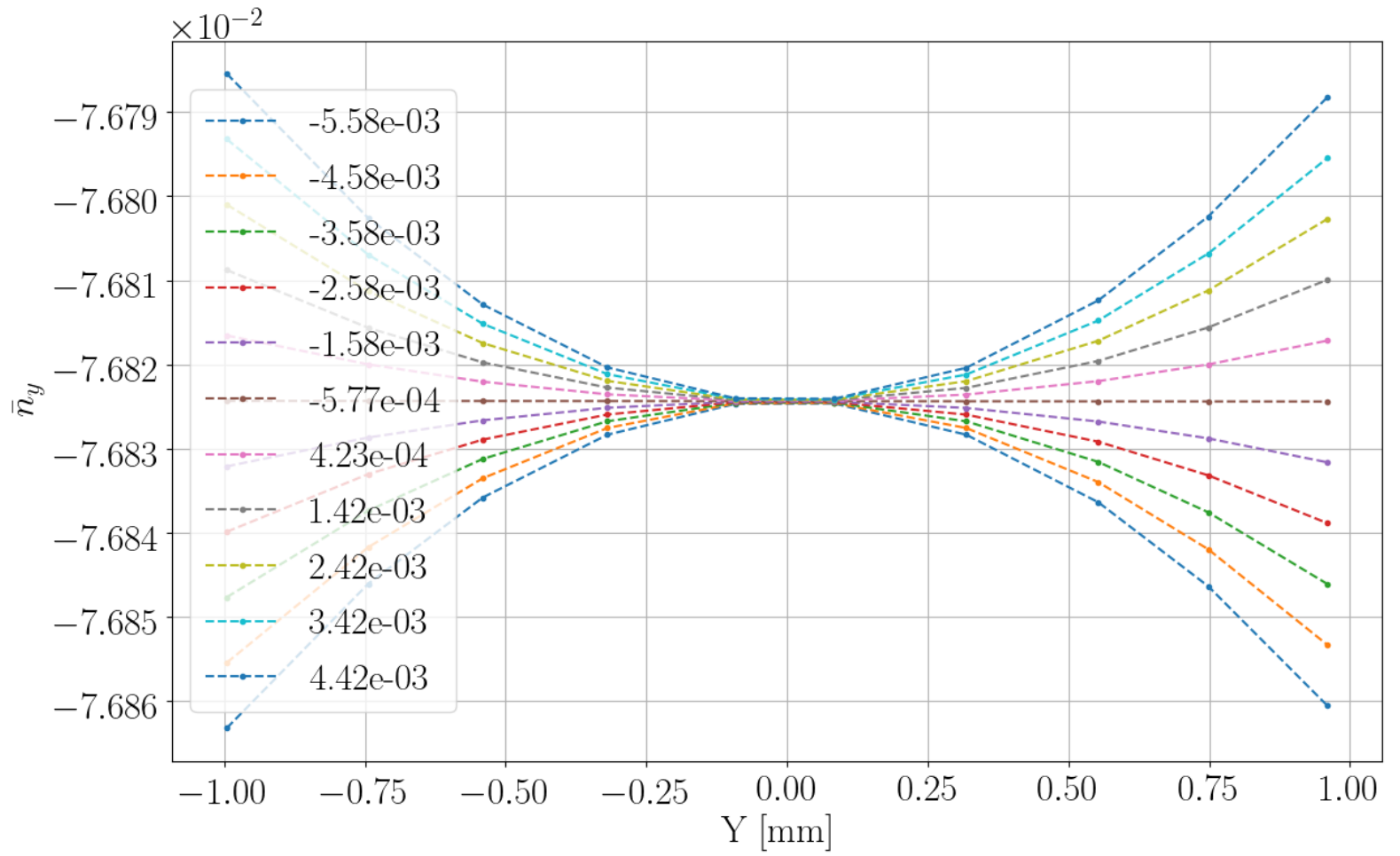

(a) Full range.

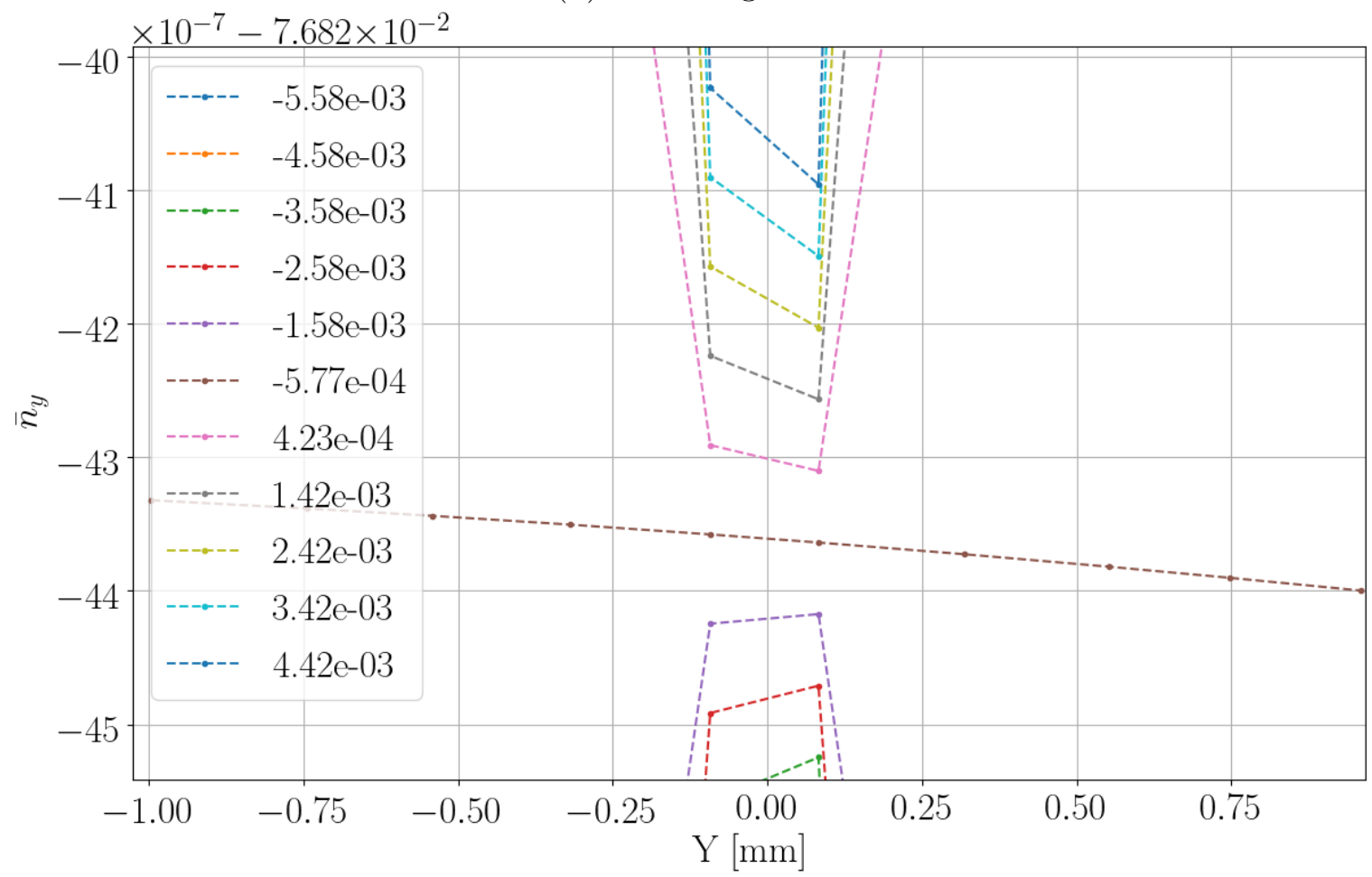

(b) Detalization.

Figure 2.7: Vertical component $\bar{n}_{y}$ of the invariant spin axis as a function of the particle's vertical offset from the closed orbit. Color marks different $G_{Y}$ values 
CHAPTER 2. UNIVERSAL SR EDM MEASUREMENT PROBLEMS AND THEIR SOLUTIONS

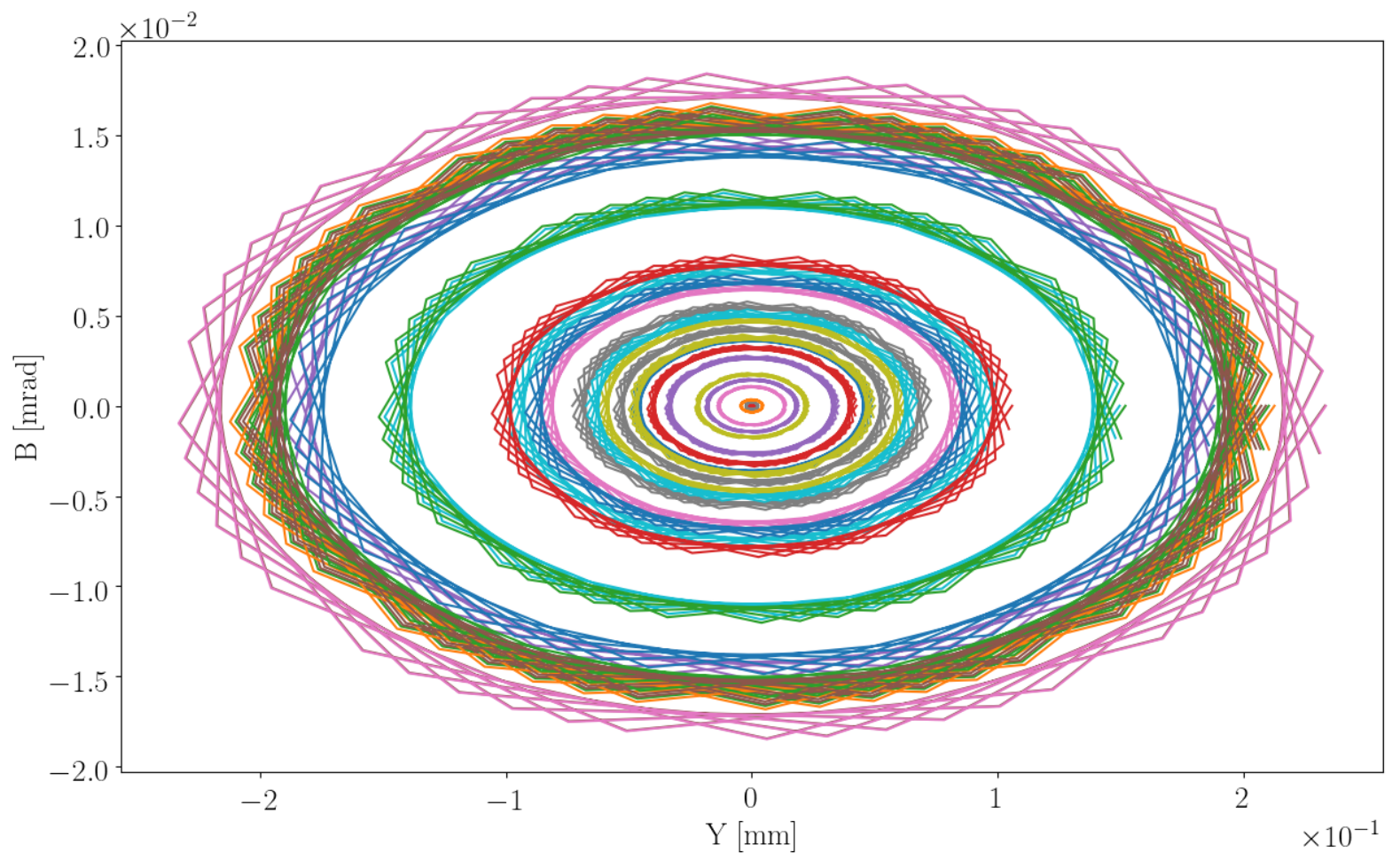

Figure 2.8: Particle trajectories in the $(Y, B)$ phase space. 
CHAPTER 2. UNIVERSAL SR EDM MEASUREMENT PROBLEMS AND THEIR SOLUTIONS

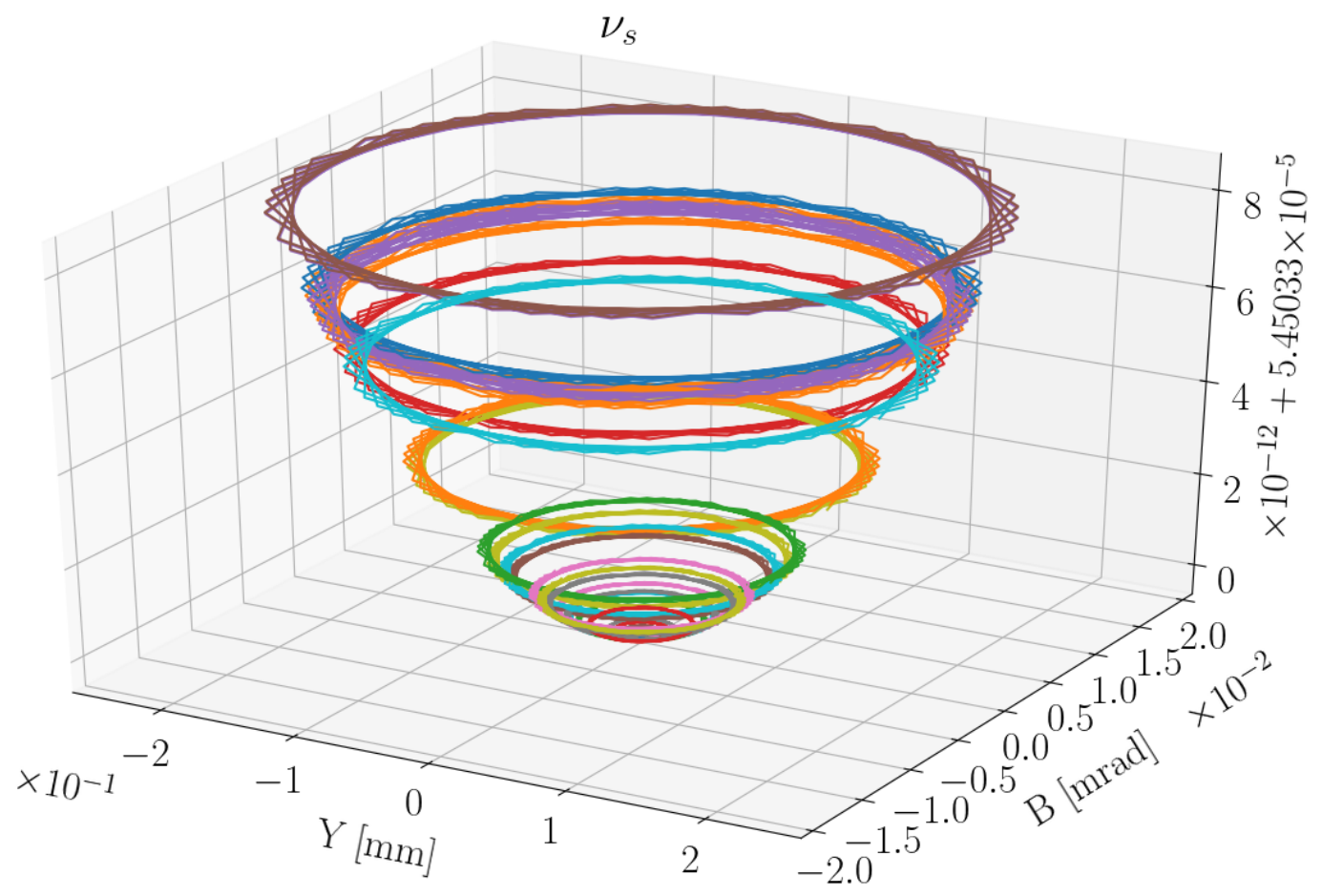

(a) Sextupoles off.

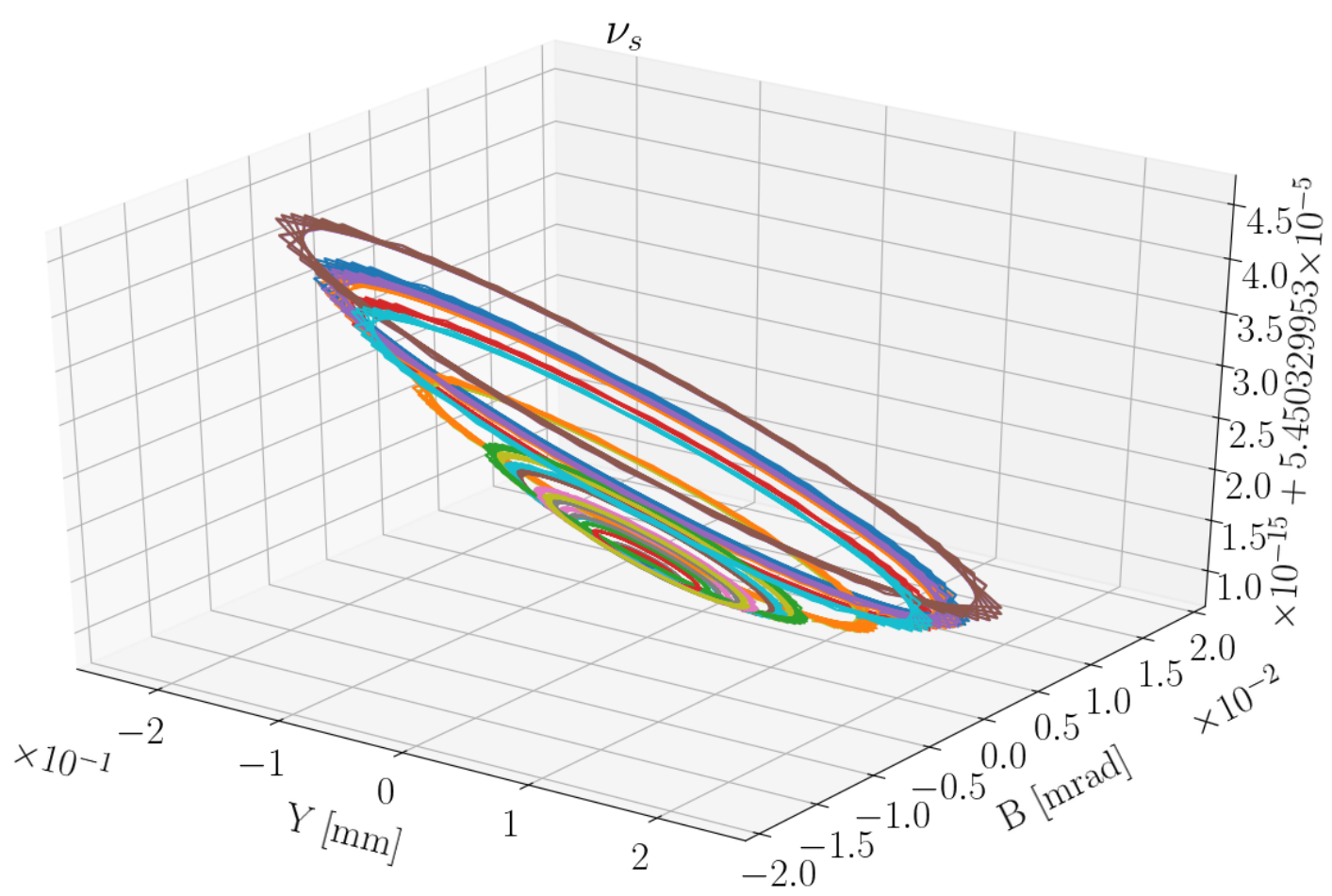

(b) Sextupoles on.

Figure 2.9: Particle spin tunes computed at their trajectories in an imperfect FS lattice. 
CHAPTER 2. UNIVERSAL SR EDM MEASUREMENT PROBLEMS AND THEIR SOLUTIONS 66

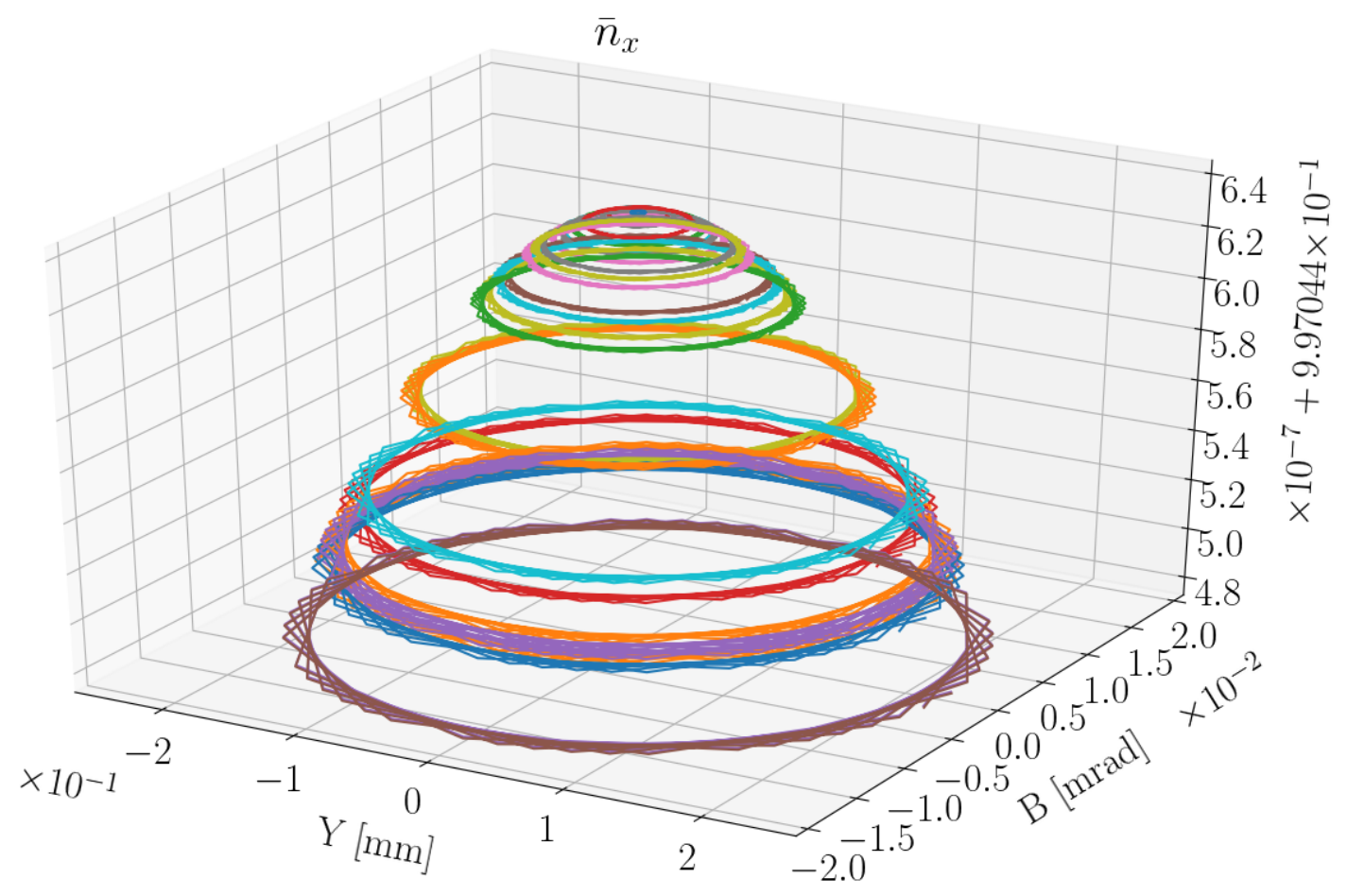

(a) Sextupoles off.

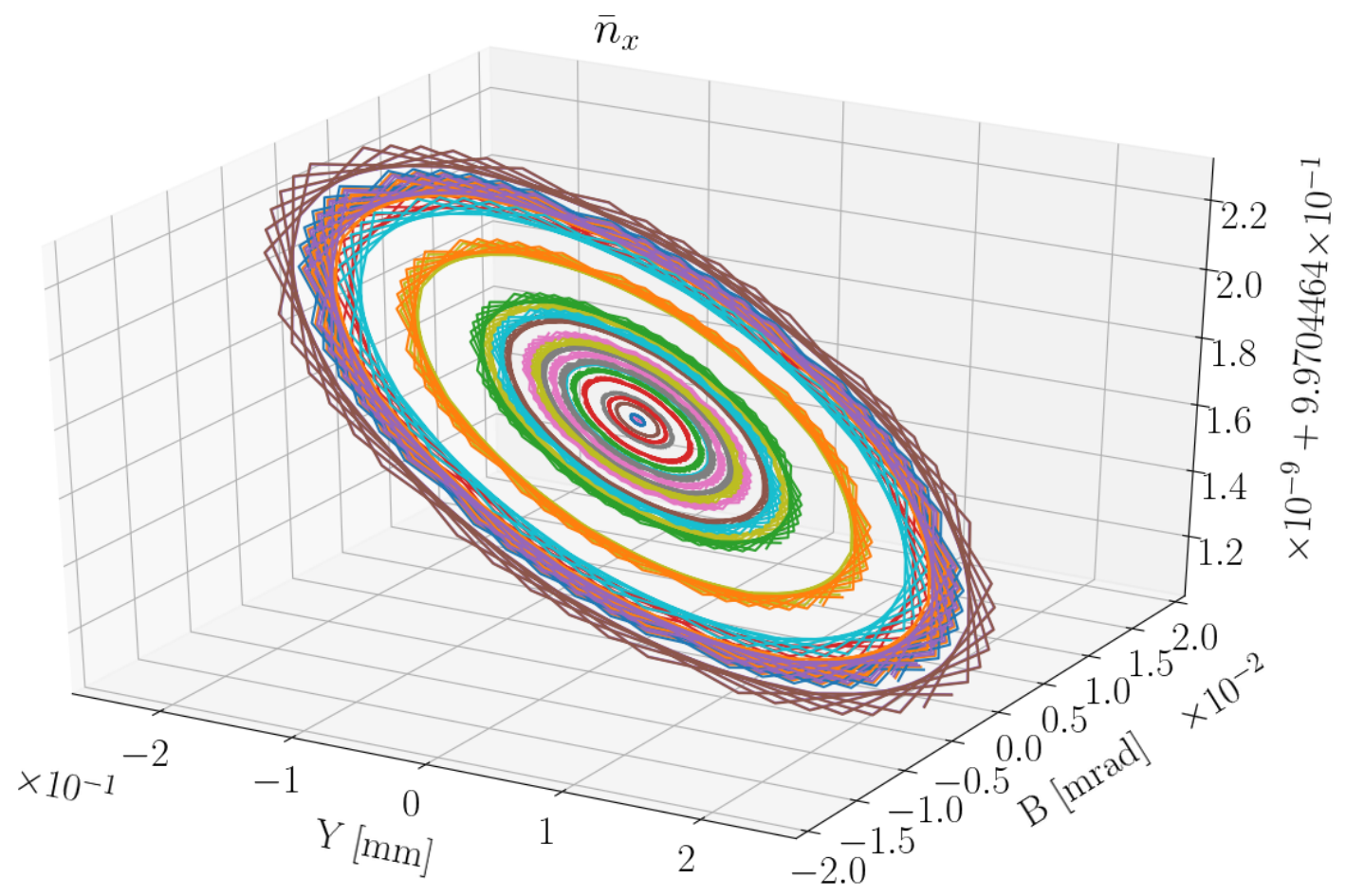

(b) Sextupoles on.

Figure 2.10: Particle's radial ISA components computed at their trajectories in an imperfect FS lattice. 
CHAPTER 2. UNIVERSAL SR EDM MEASUREMENT PROBLEMS AND THEIR SOLUTIONS

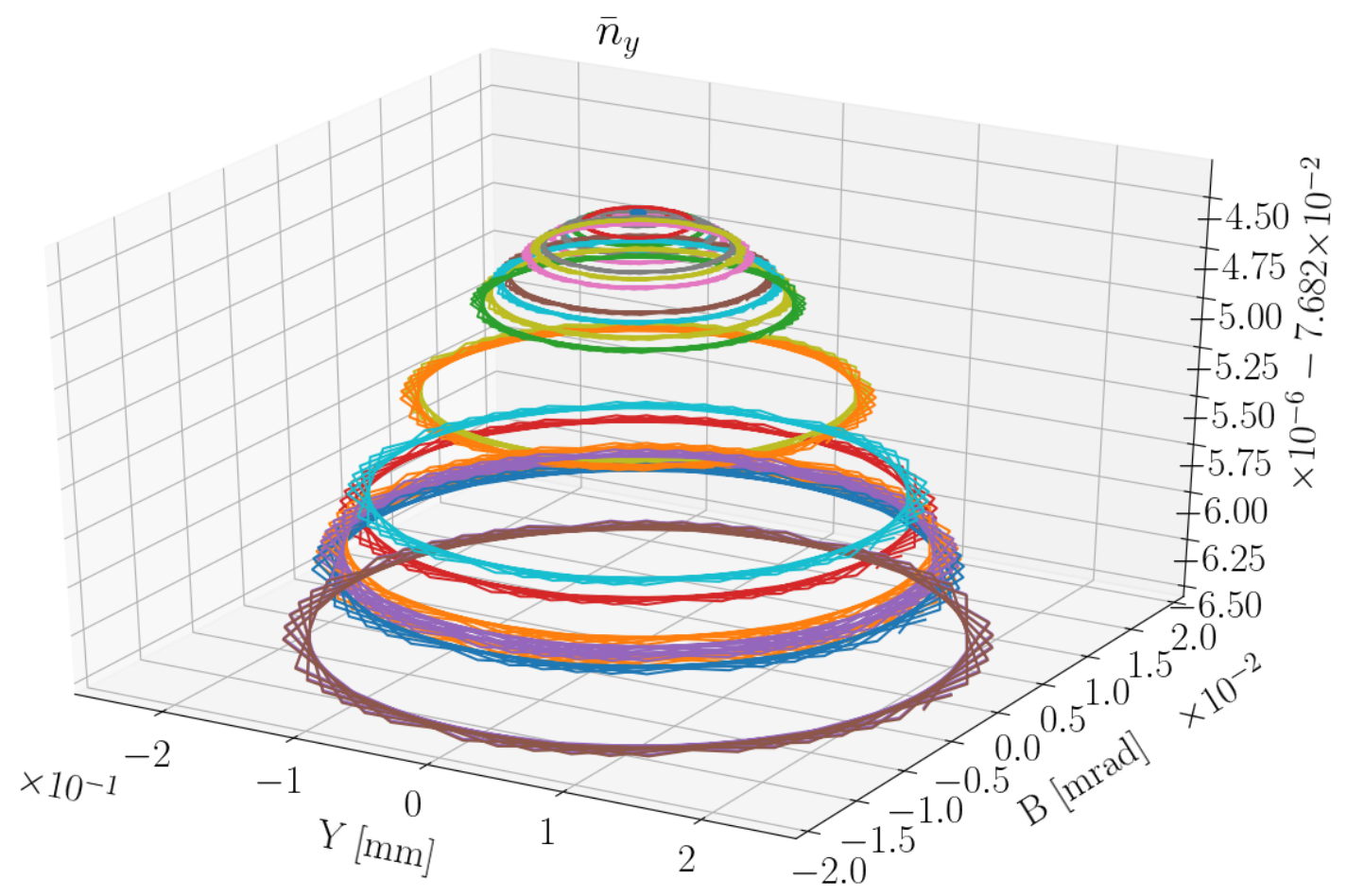

(a) Sextupoles off.

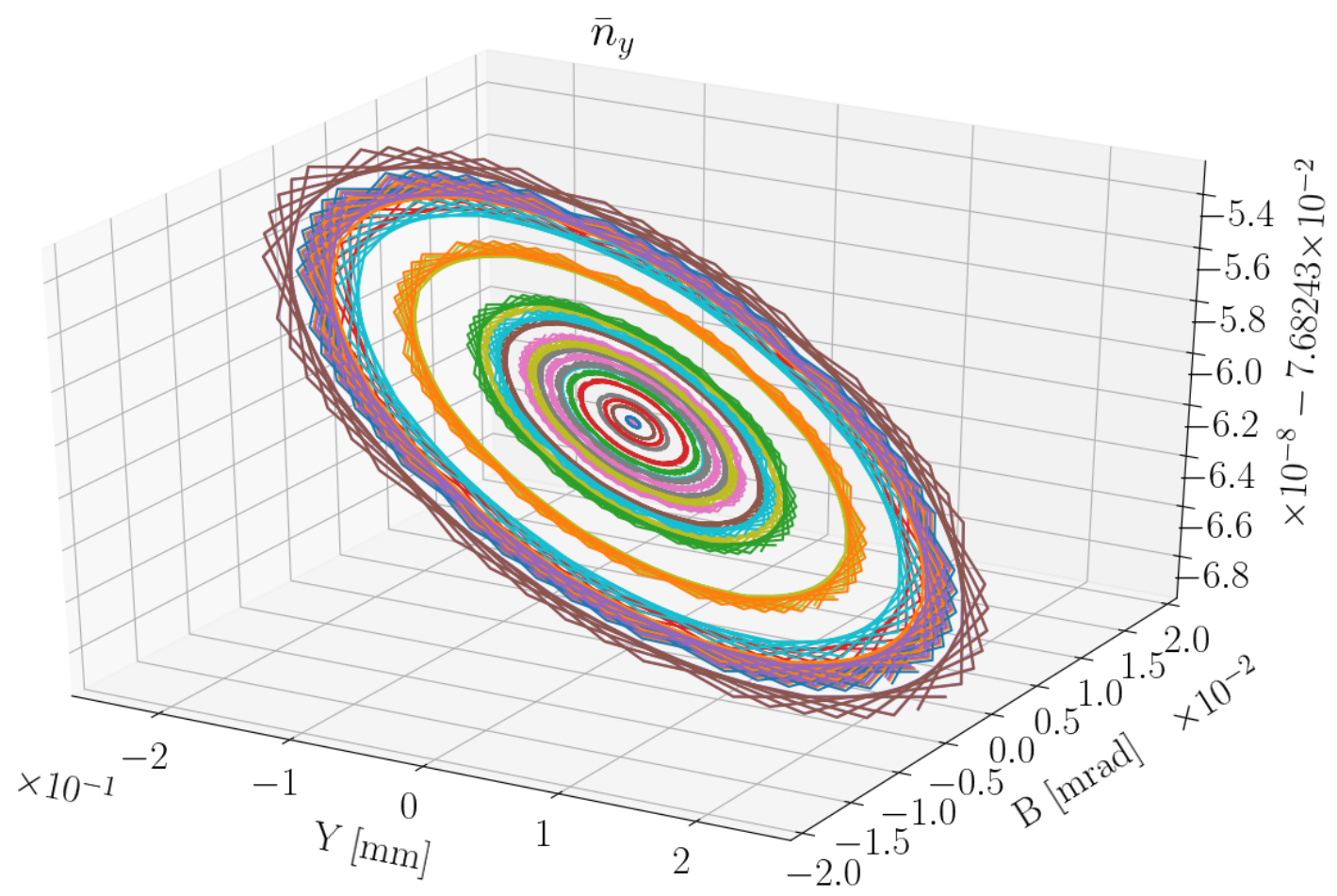

(b) Sextupoles on.

Figure 2.11: Particle's vertical ISA components computed at their trajectories in an imperfect FS lattice. 
CHAPTER 2. UNIVERSAL SR EDM MEASUREMENT PROBLEMS AND THEIR SOLUTIONS 68

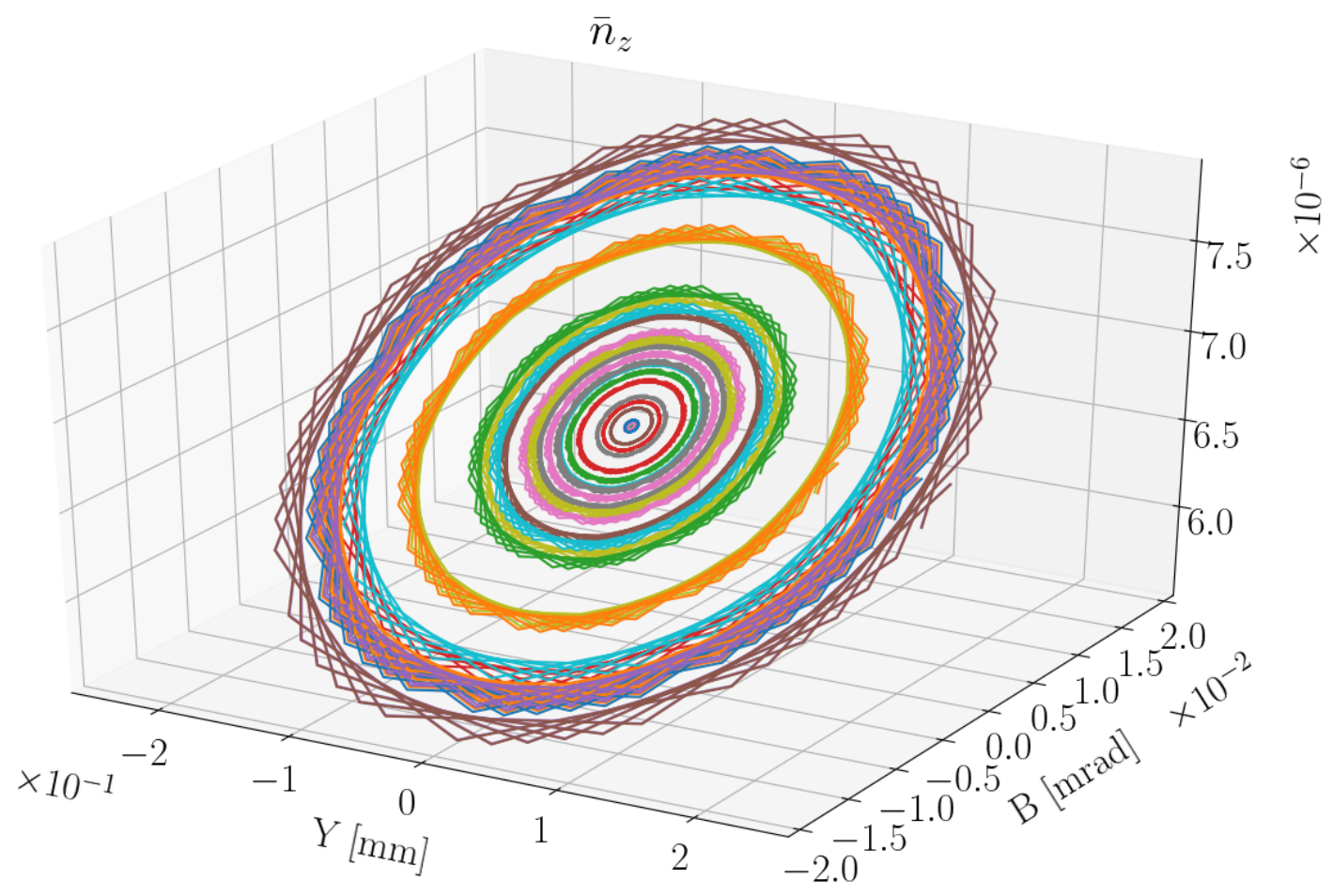

(a) Sextupoles off.

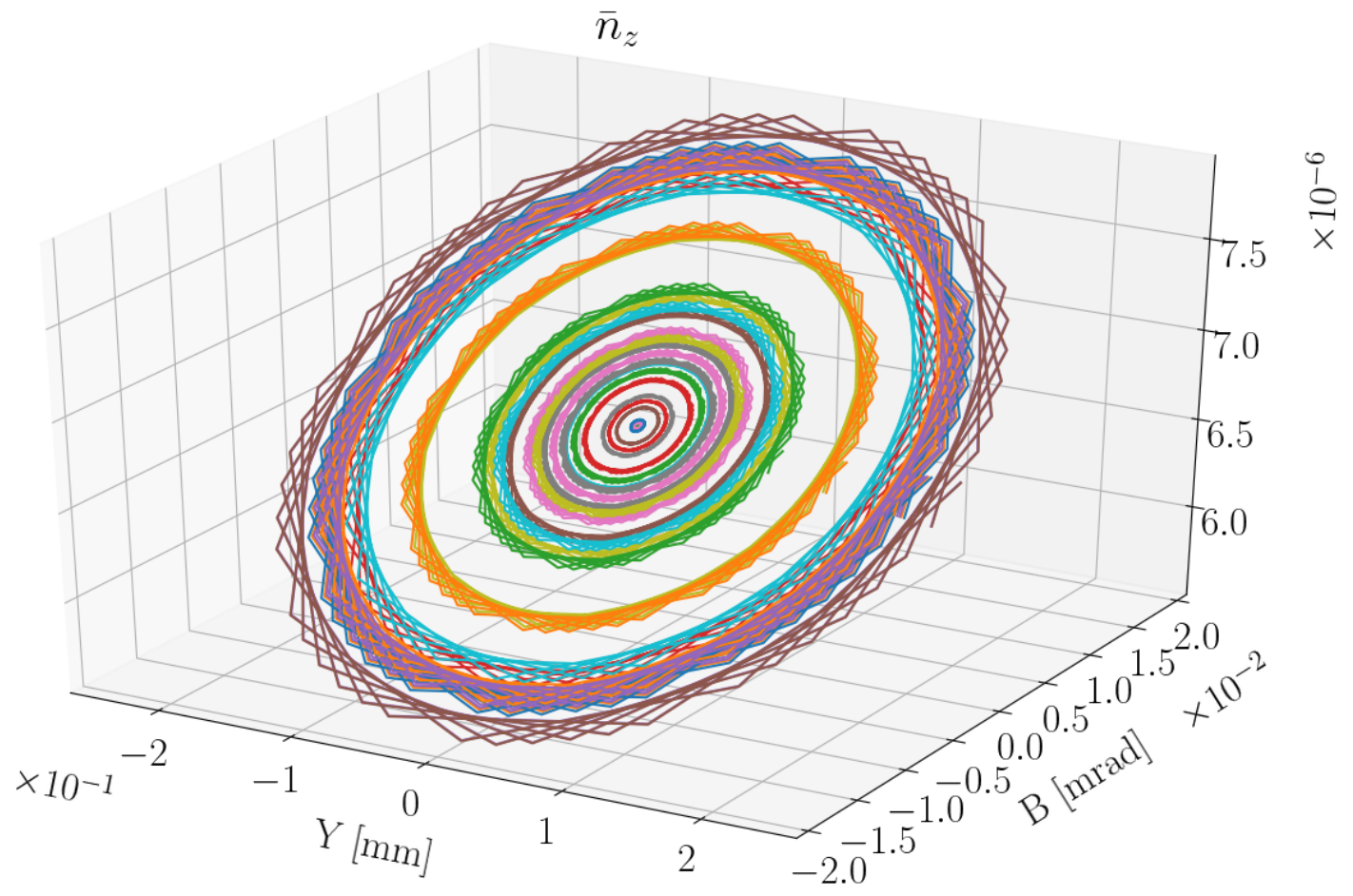

(b) Sextupoles on.

Figure 2.12: Particle's longitudinal ISA components computed at their trajectories in an imperfect FS lattice. 
CHAPTER 2. UNIVERSAL SR EDM MEASUREMENT PROBLEMS AND THEIR SOLUTIONS
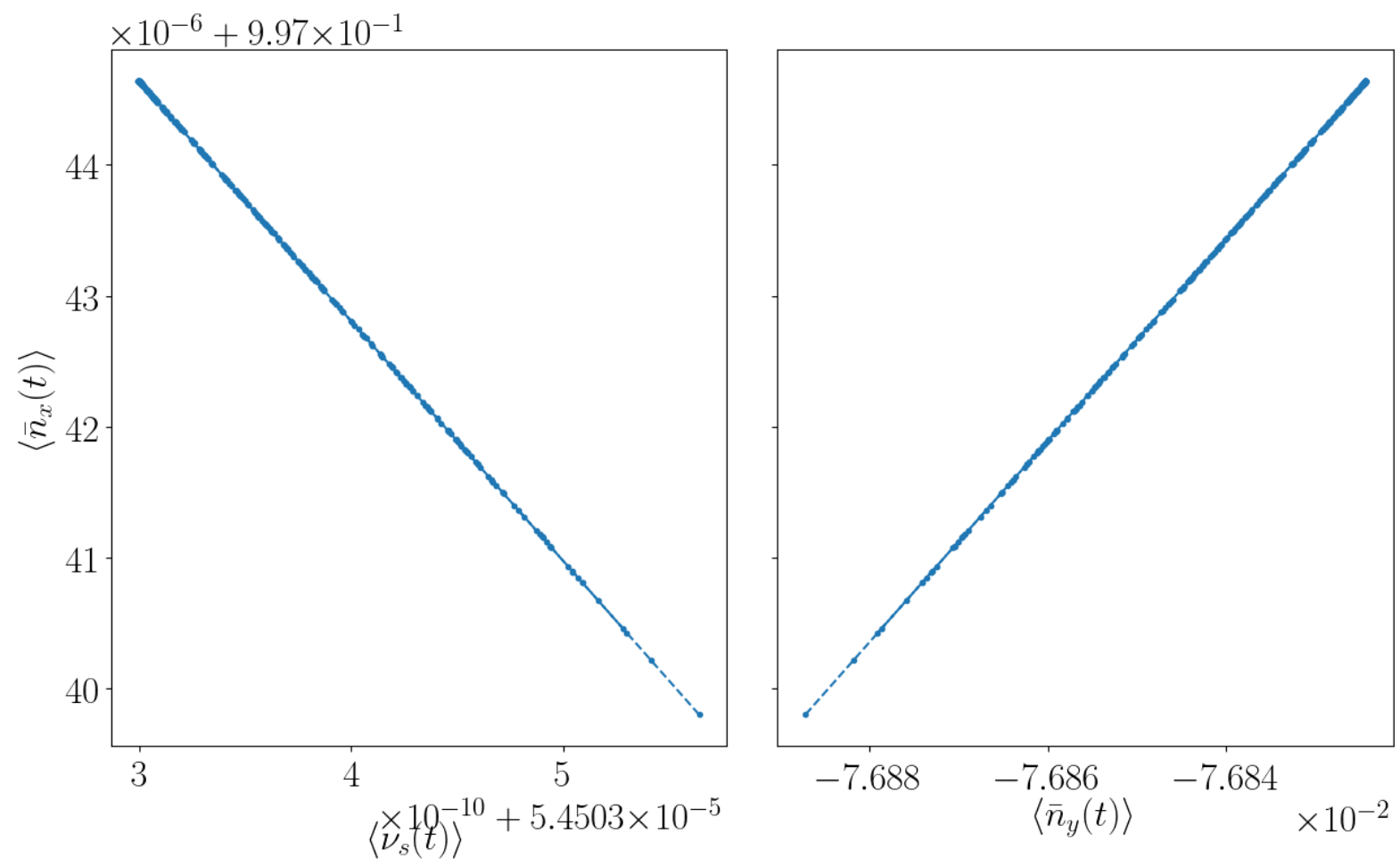

Figure 2.13: Mean level of the radial and vertical ISA components versus the correcponding value of spin tune. 
CHAPTER 2. UNIVERSAL SR EDM MEASUREMENT PROBLEMS AND THEIR SOLUTIONS
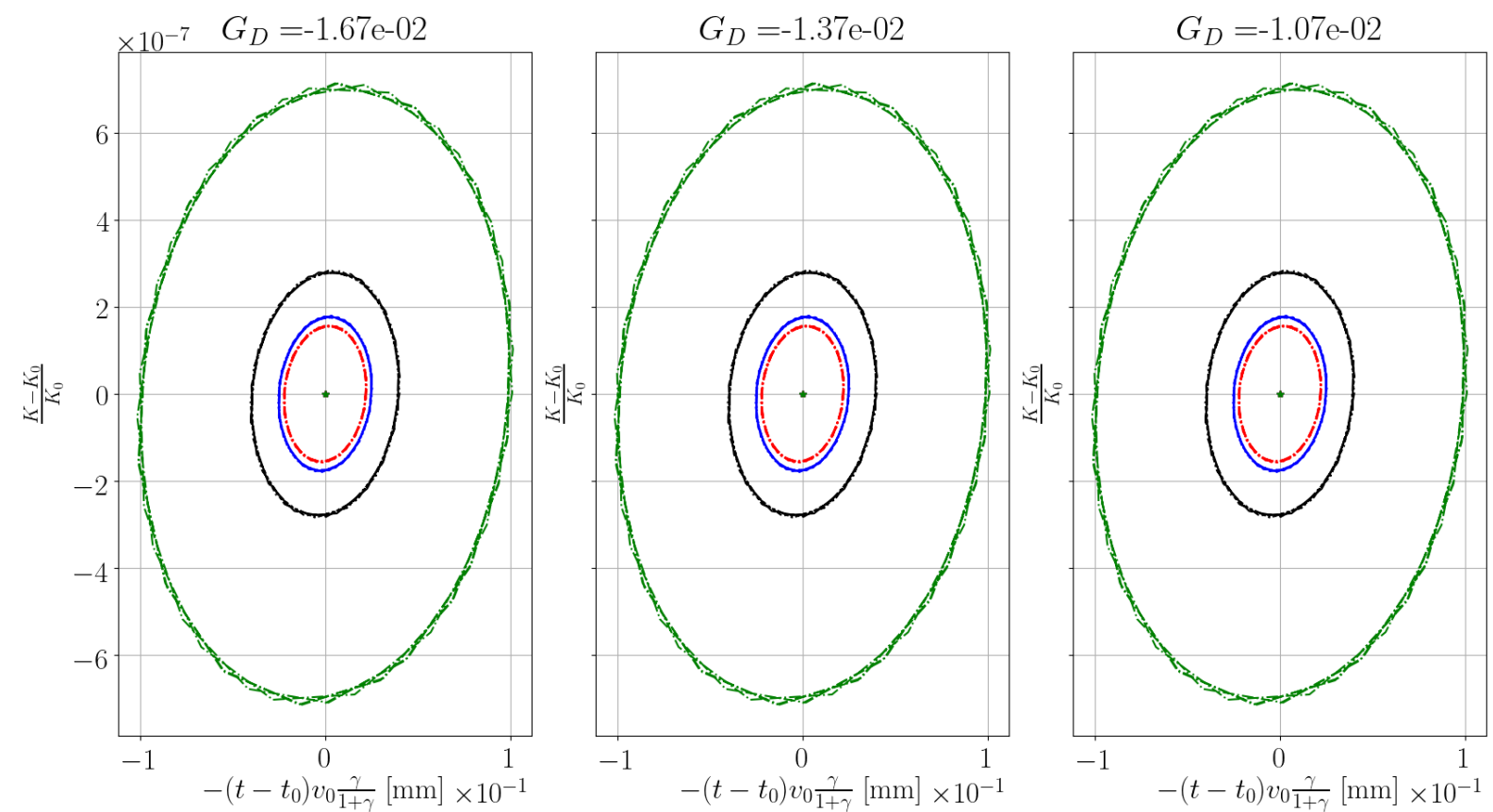

(a) D-bunch phase portraits.
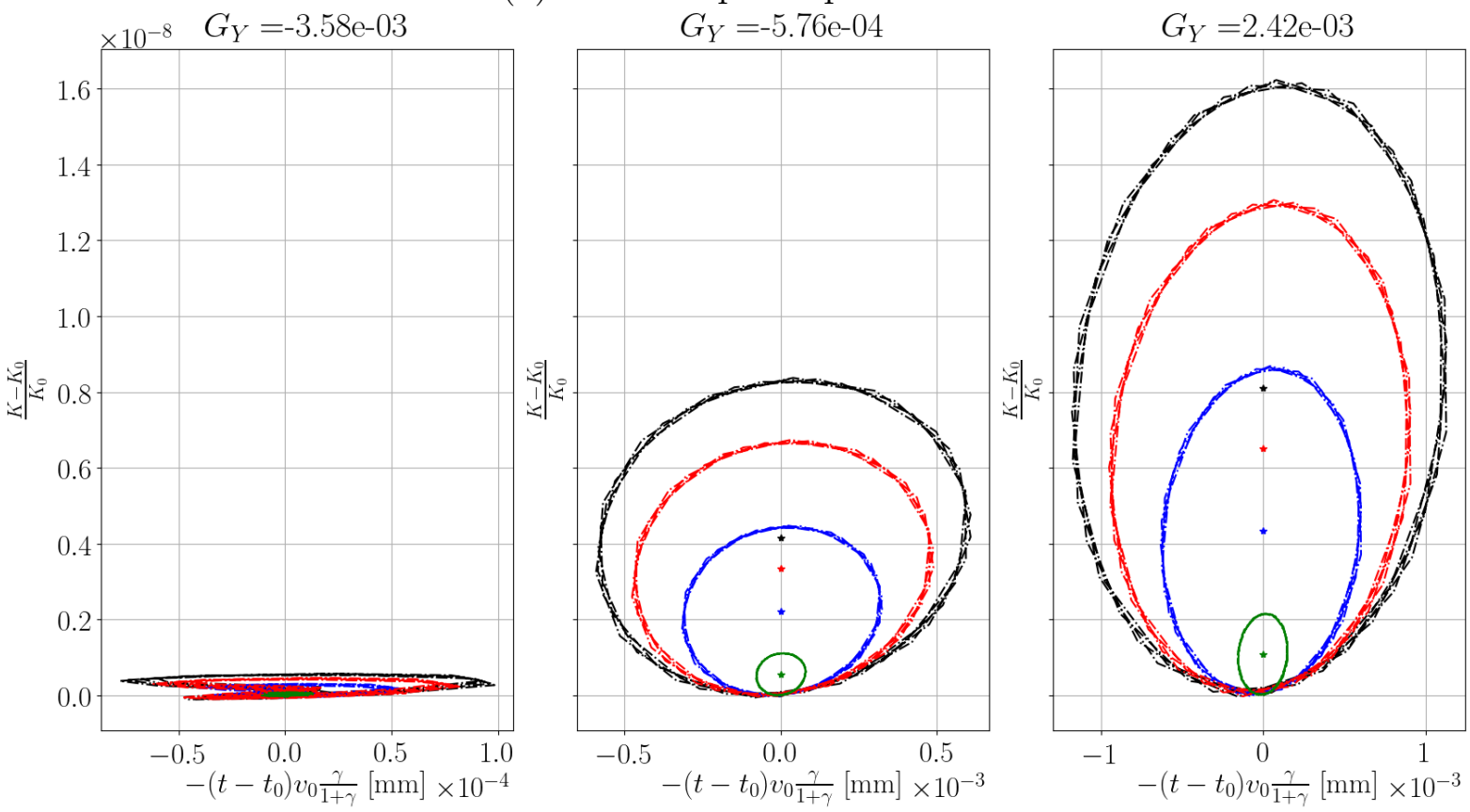

(b) Y-bunch phase portraits.

Figure 2.14: Longitudinal phase space particle portraits. Asterisks mark the ellipse centers. Colors mark trajectories of particles with differing initial vertical offset from the reference orbit. 
CHAPTER 2. UNIVERSAL SR EDM MEASUREMENT PROBLEMS AND THEIR SOLUTIONS

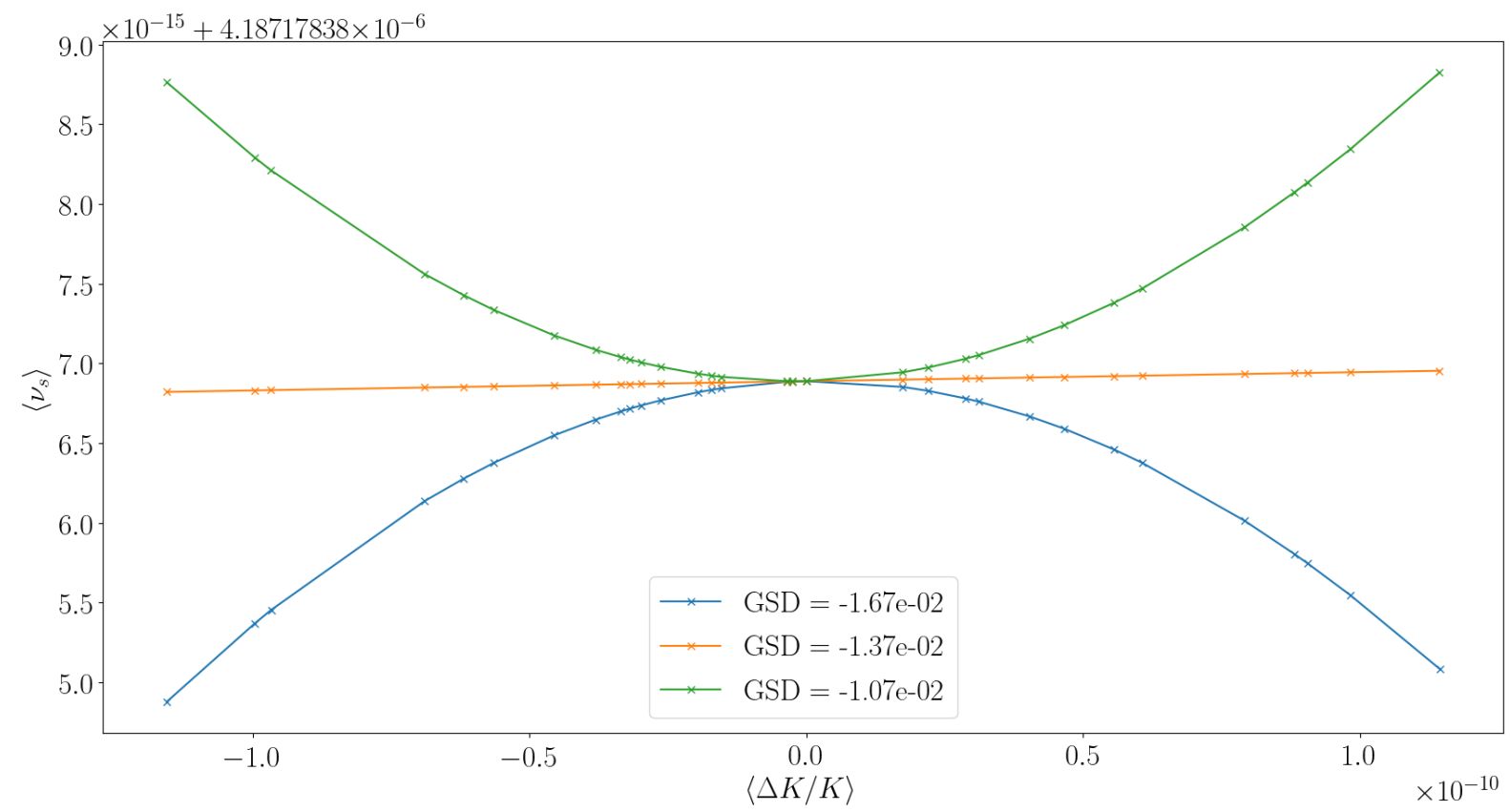

(a) For the D-bunch.

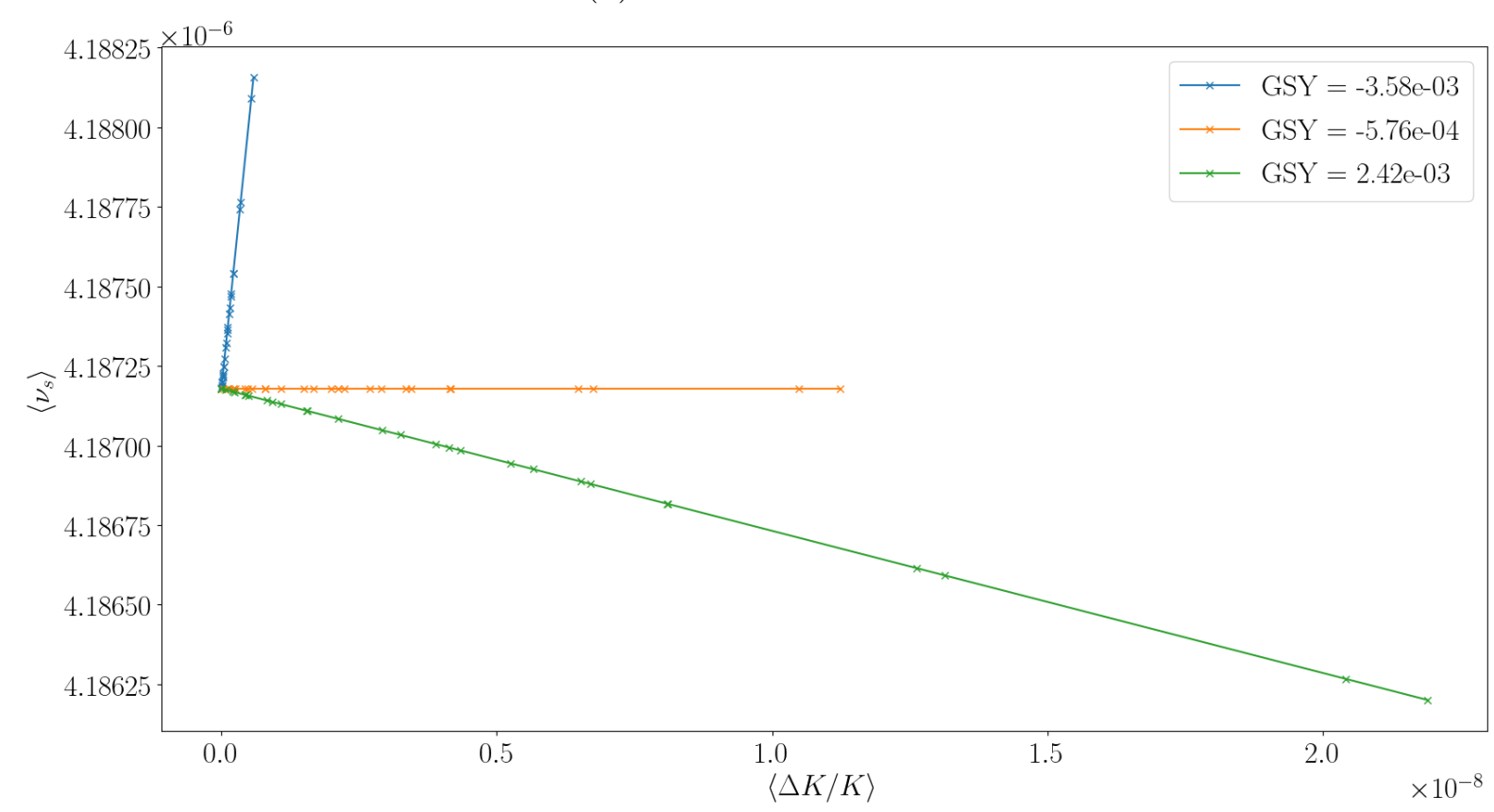

(b) For the Y-bunch.

Figure 2.15: Particle mean spin tune level as a function of its equilibrium level energy at different sextupole strengths. 
CHAPTER 2. UNIVERSAL SR EDM MEASUREMENT PROBLEMS AND THEIR SOLUTIONS

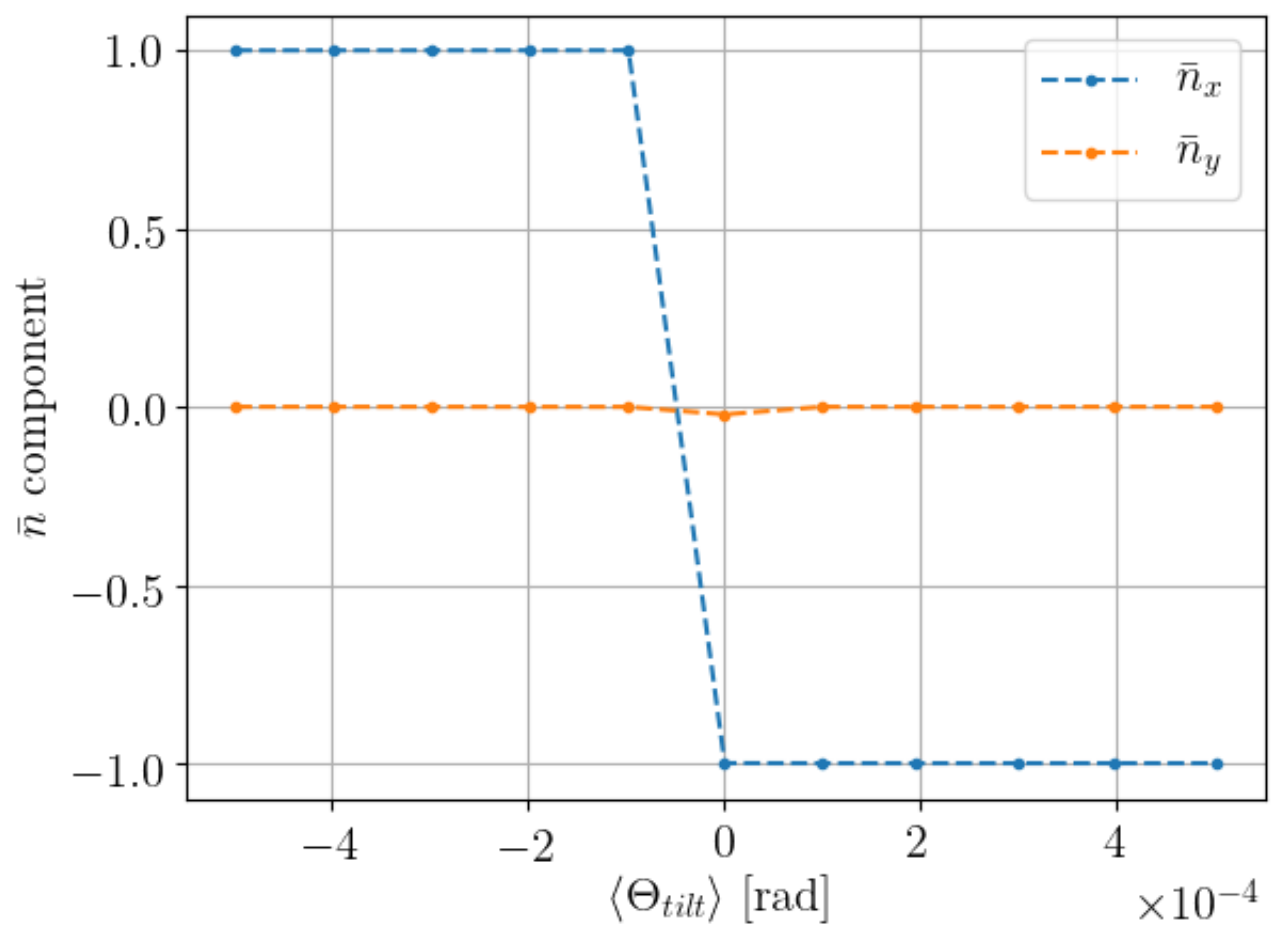

(a) Spin precession axis $\bar{n}$ components.

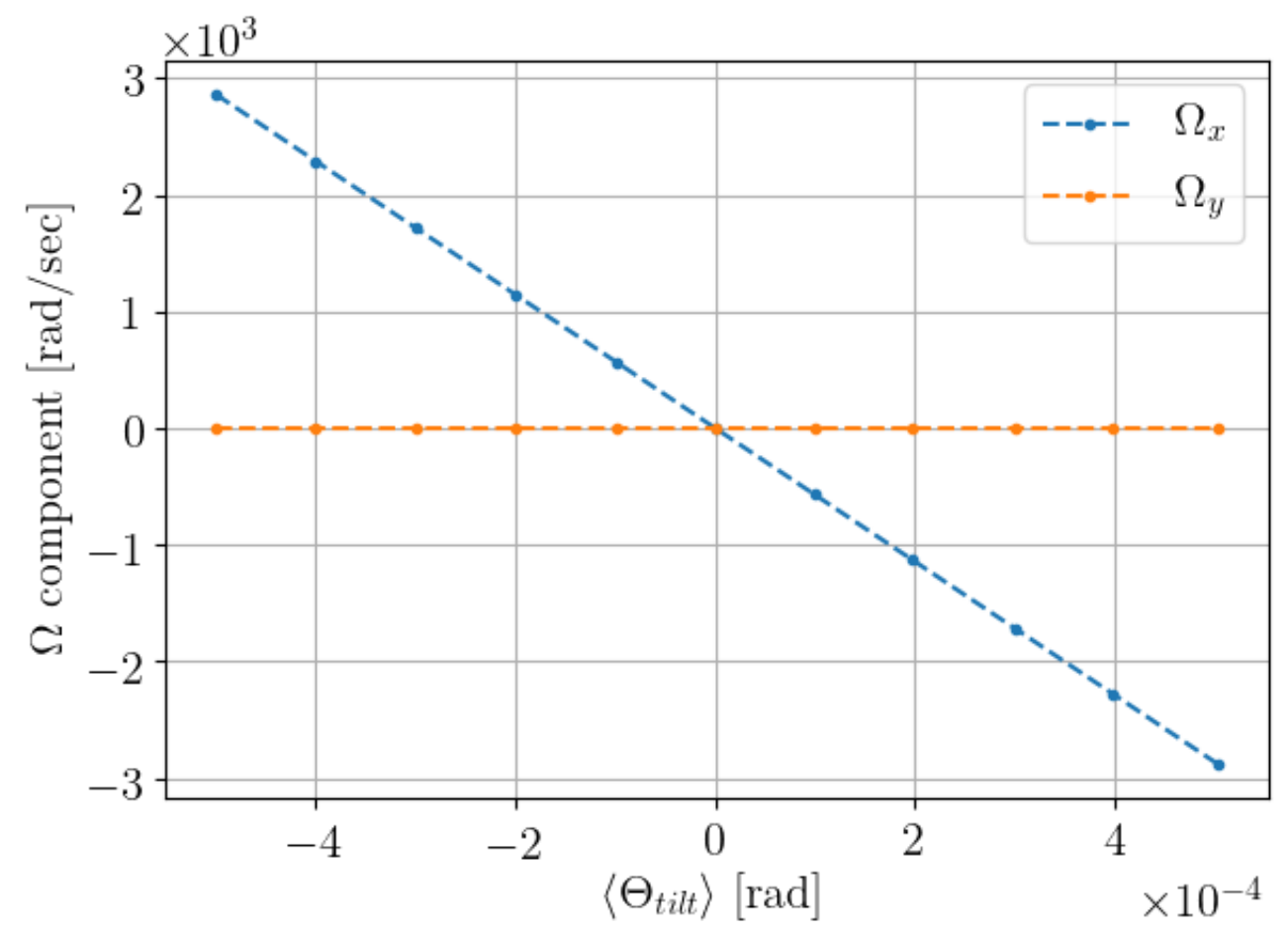

(b) Angular velocity $\Omega$ components.

Figure 2.16: Reference particle's spin precession axis and angular velocity components as functions of the mean $\mathrm{E}+\mathrm{B}$ element tilt angle. Element tilts are normally distributed. Color identifies the component; radial (blue) and vertical (orange). 
CHAPTER 2. UNIVERSAL SR EDM MEASUREMENT PROBLEMS AND THEIR SOLUTIONS

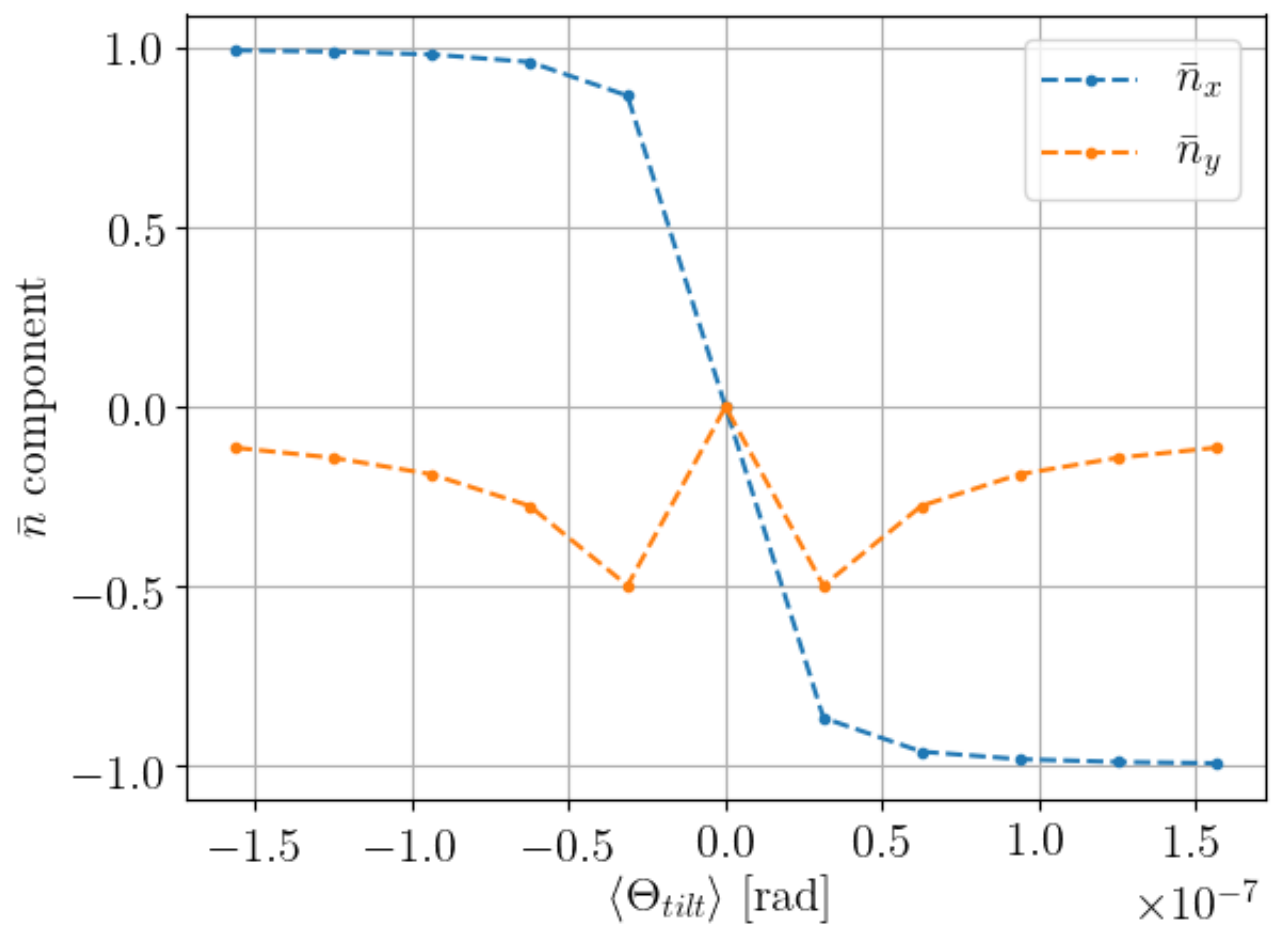

(a) Spin precession axis $\bar{n}$ components.

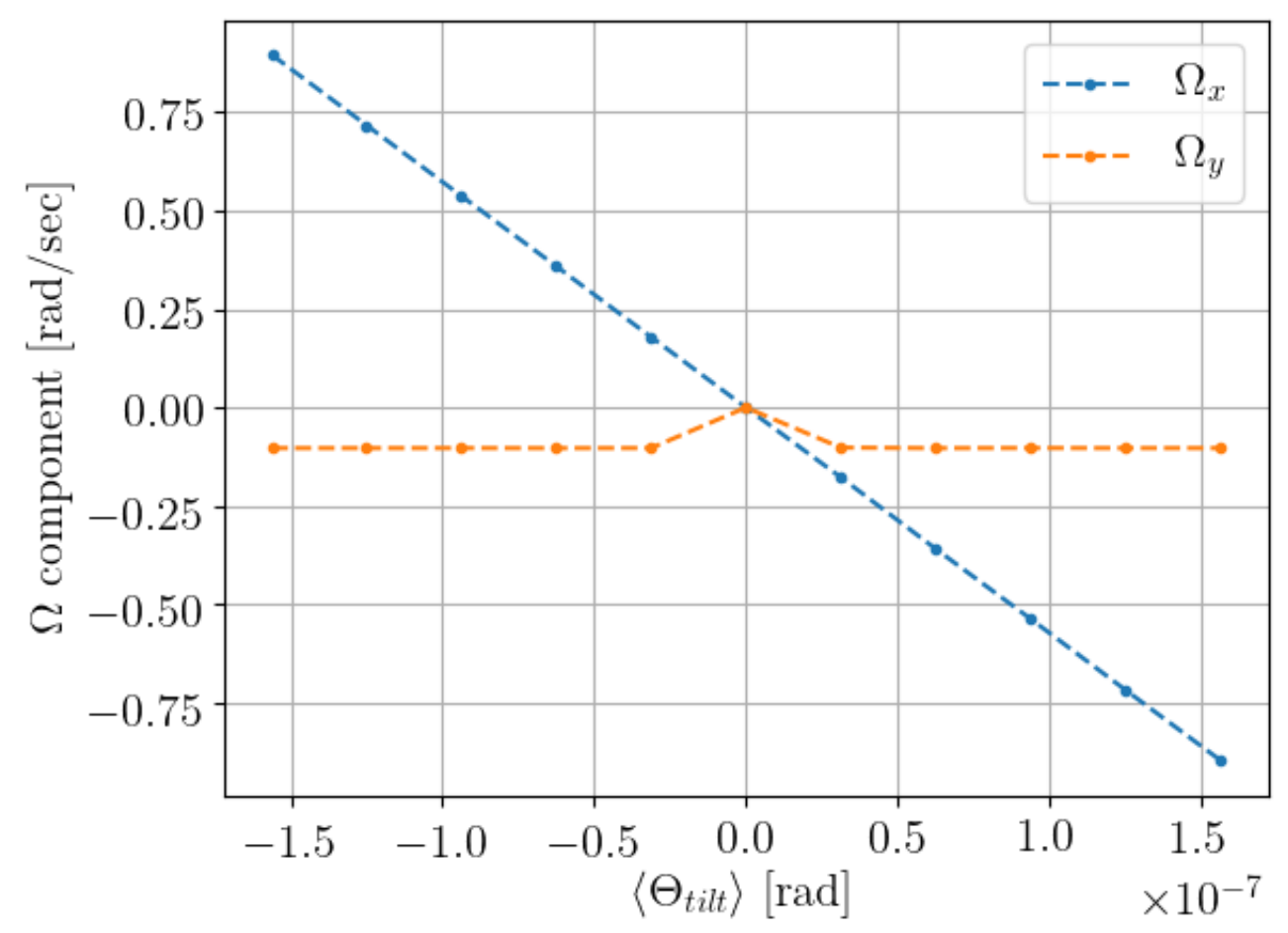

(b) Angular velocity vector $\Omega$ components.

Figure 2.17: Reference particle's spin precession axis and angular velocity components as functions of the mean $\mathrm{E}+\mathrm{B}$ element tilt angle. Three mutually-compensated tilt pairs plus an uncompensated rotation. Color identifies the component; radial (blue) and vertical (orange) 
CHAPTER 2. UNIVERSAL SR EDM MEASUREMENT PROBLEMS AND THEIR SOLUTIONS

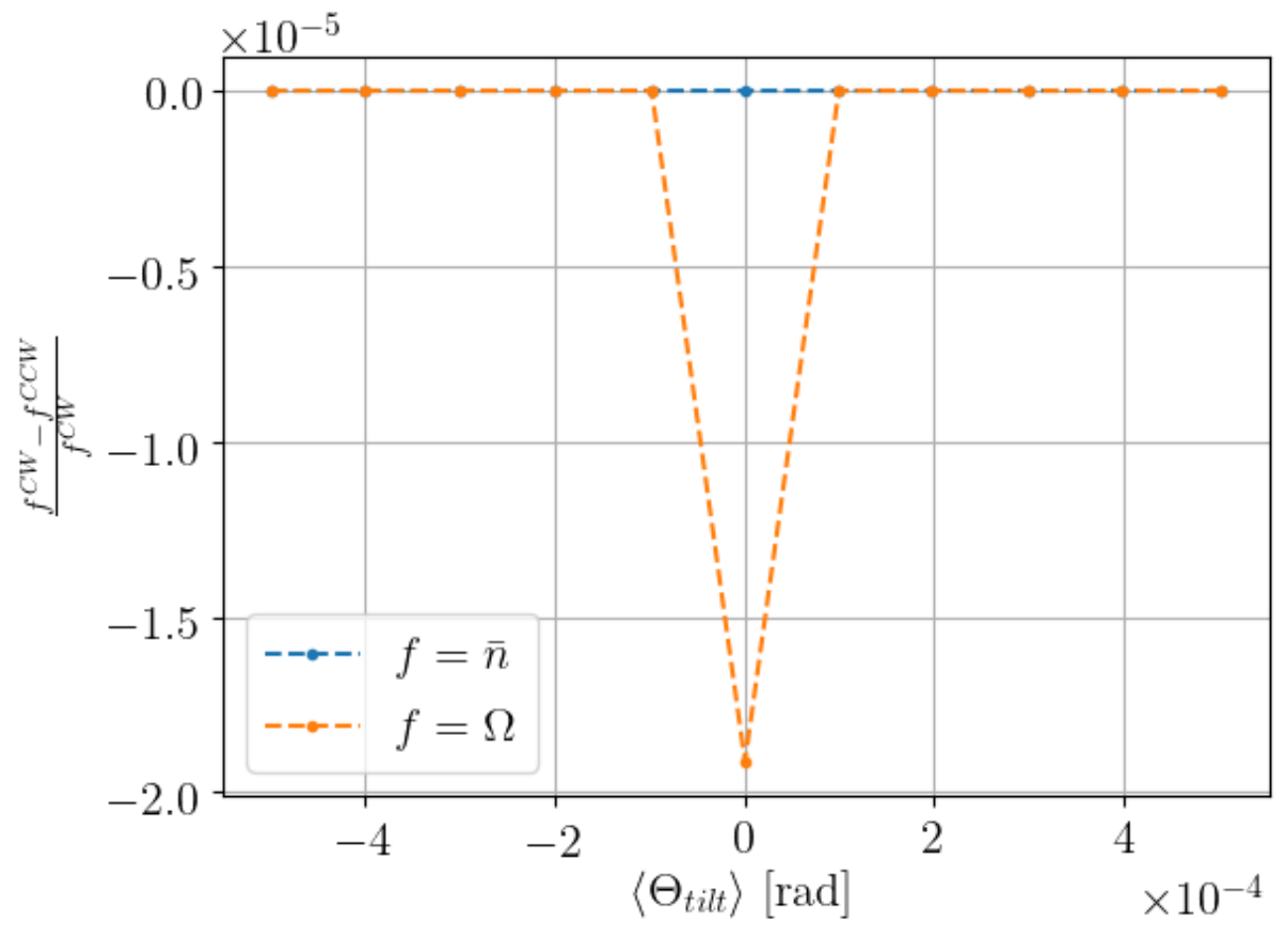

(a) Normally distributed E+B element tilts.

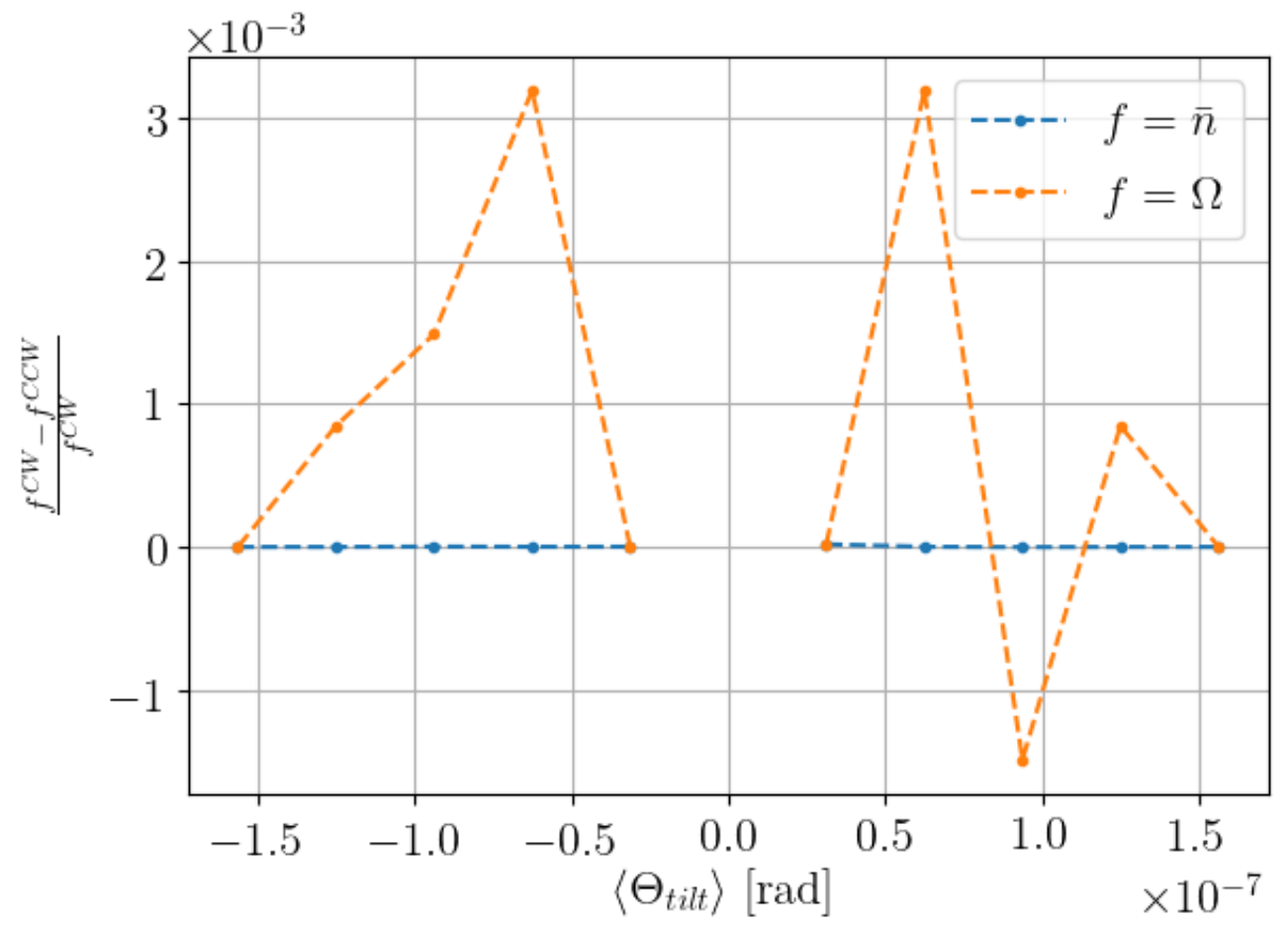

(b) Mutually-compensated element tilts.

Figure 2.18: Relative difference between the CW and CCW beams' spin precession axis and angular velocity radial components. Color marks the compared variable: spin precession axis (blue) and abgular velocity (orange). 
CHAPTER 2. UNIVERSAL SR EDM MEASUREMENT PROBLEMS AND THEIR SOLUTIONS
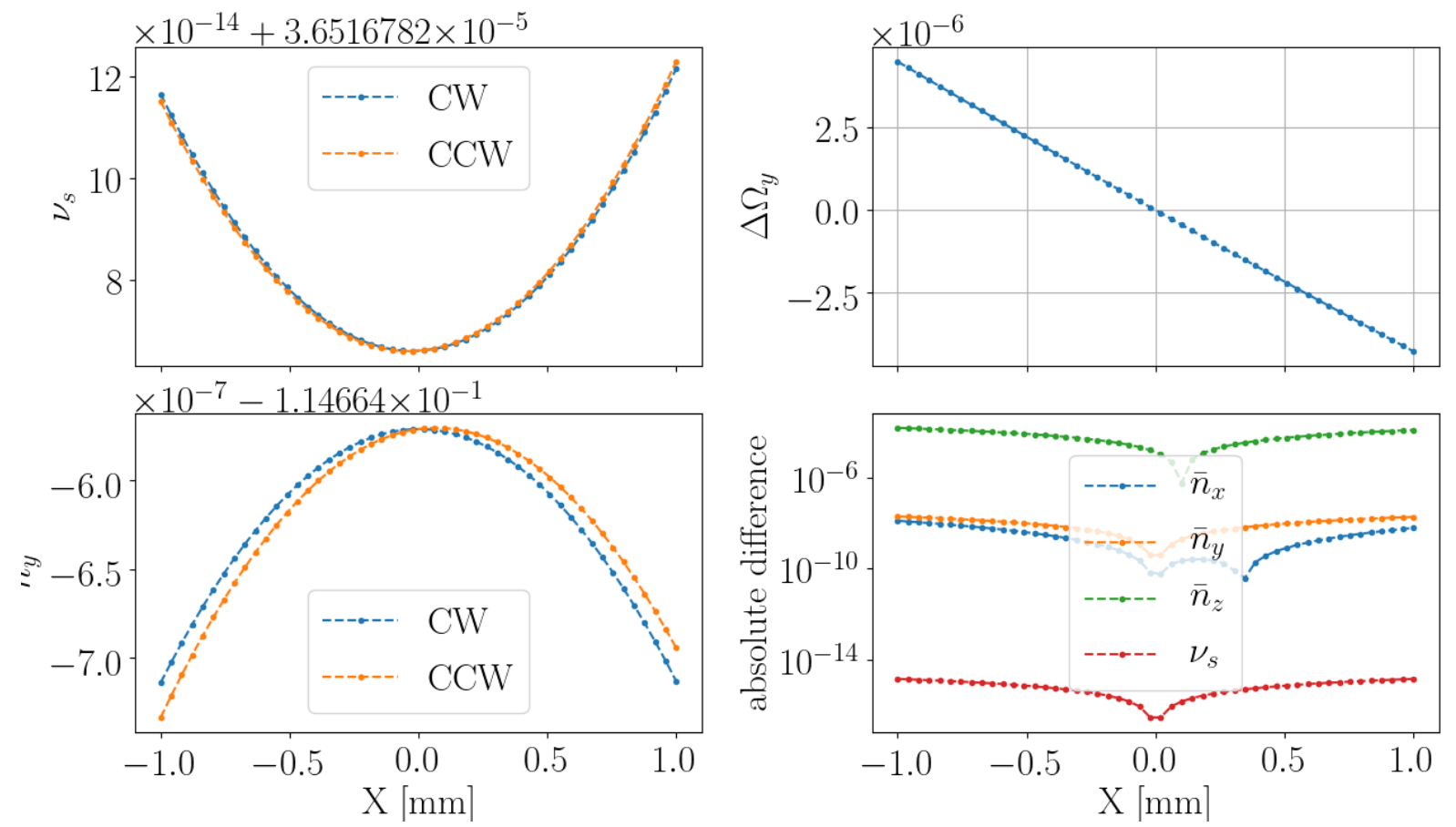

(a) Spin tune and invariant spin axis as functions of the particle's horizontal offset from the closed orbit.

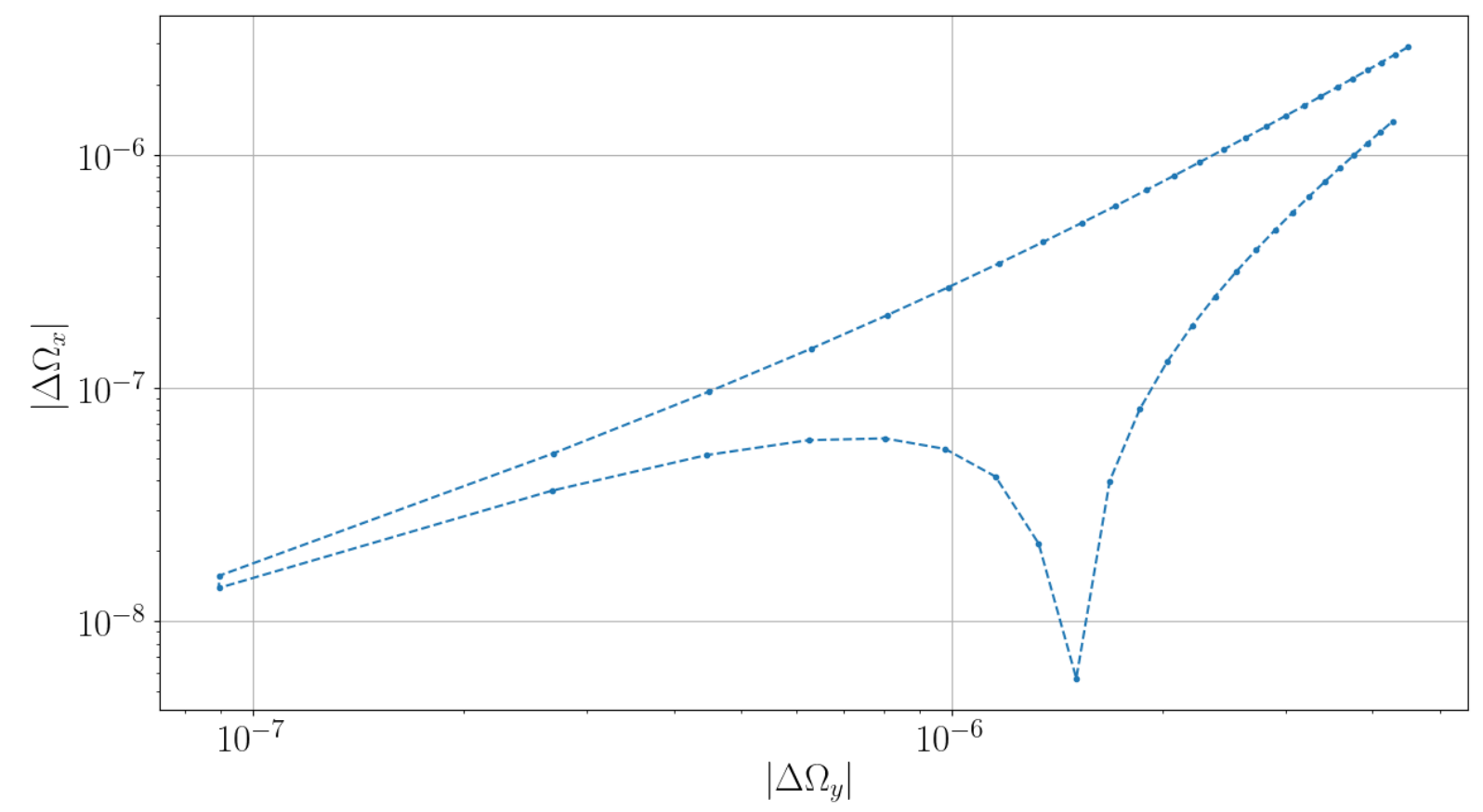

(b) Difference between the CW \& CCW beams' radial spin precession angular velocity components as a function of the differnce between their vertical components (calibration plot).

Figure 2.19: Simulation results in the case fo horizontal plane betatron motion-related spin decoherence. 
CHAPTER 2. UNIVERSAL SR EDM MEASUREMENT PROBLEMS AND THEIR SOLUTIONS
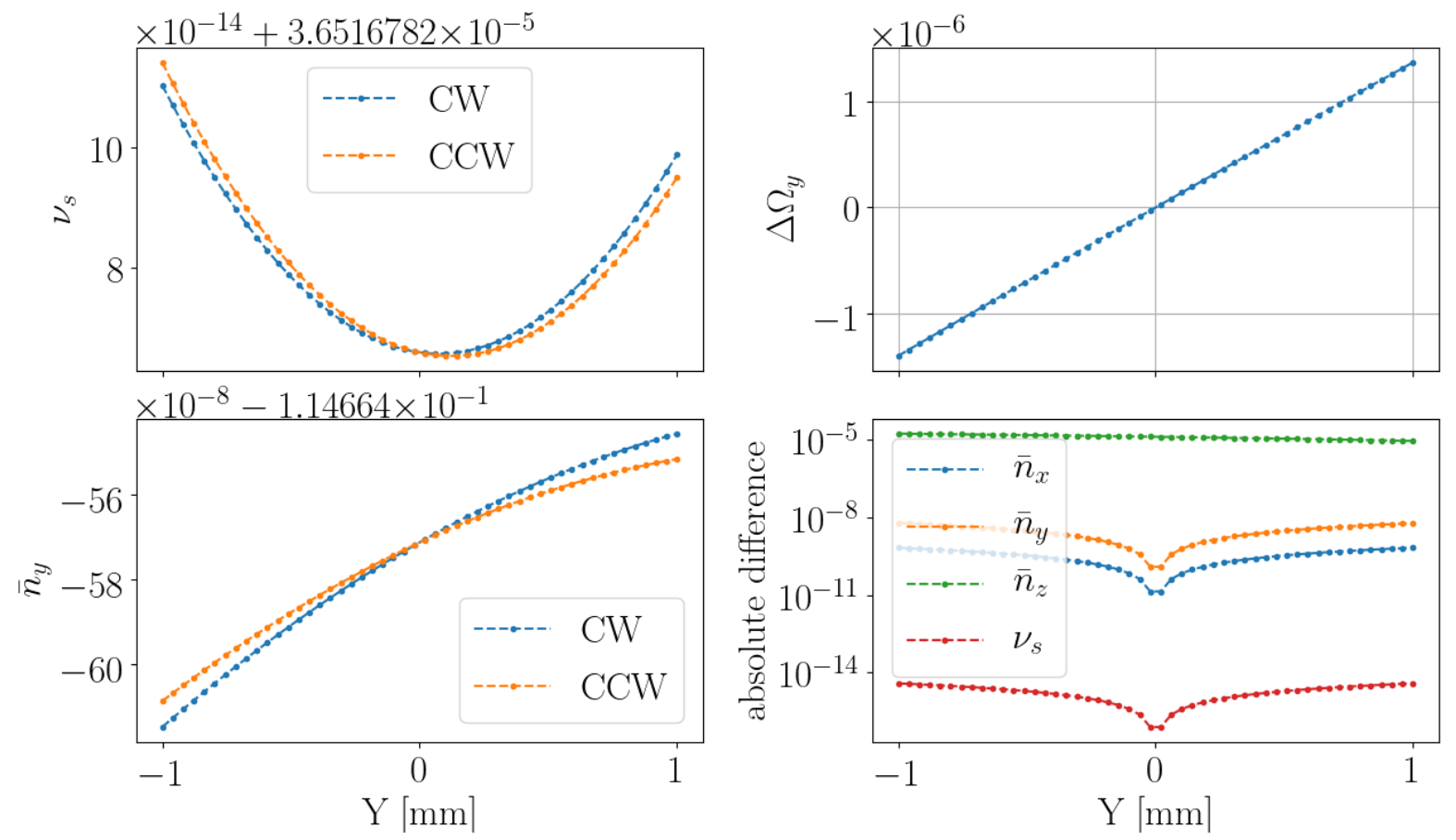

(a) Spin tune and invariant spin axis as functions of the particle's vertical offset from the closed orbit.

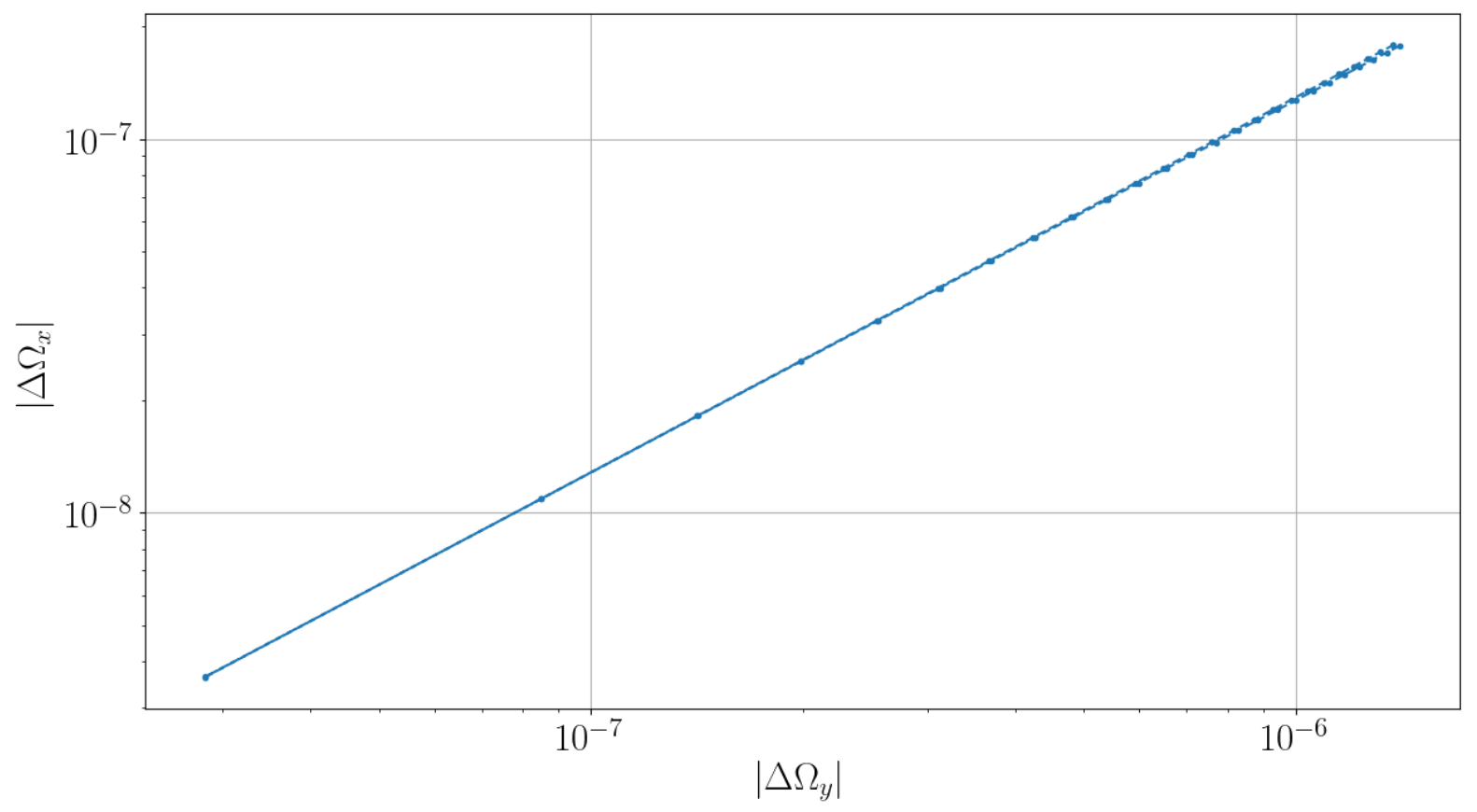

(b) Difference between the CW \& CCW beams' radial spin precession angular velocity components as a function of the differnce between their vertical components (calibration plot).

Figure 2.20: Simulation results in the case fo vertical plane betatron motion-related spin decoherence. 
CHAPTER 2. UNIVERSAL SR EDM MEASUREMENT PROBLEMS AND THEIR SOLUTIONS
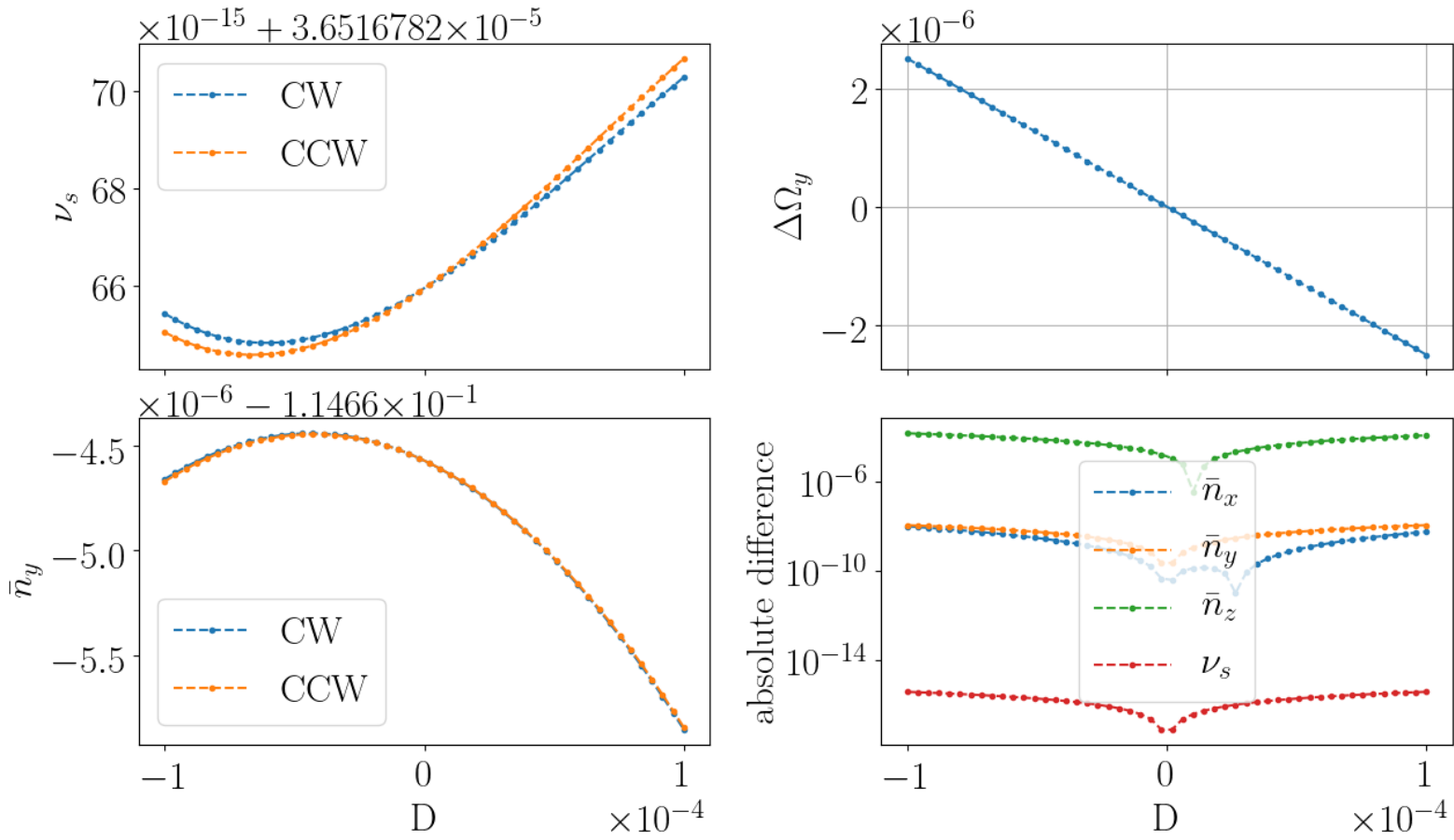

(a) Spin tune and invariant spin axis as functions of the particle's energy offset from the reference energy.

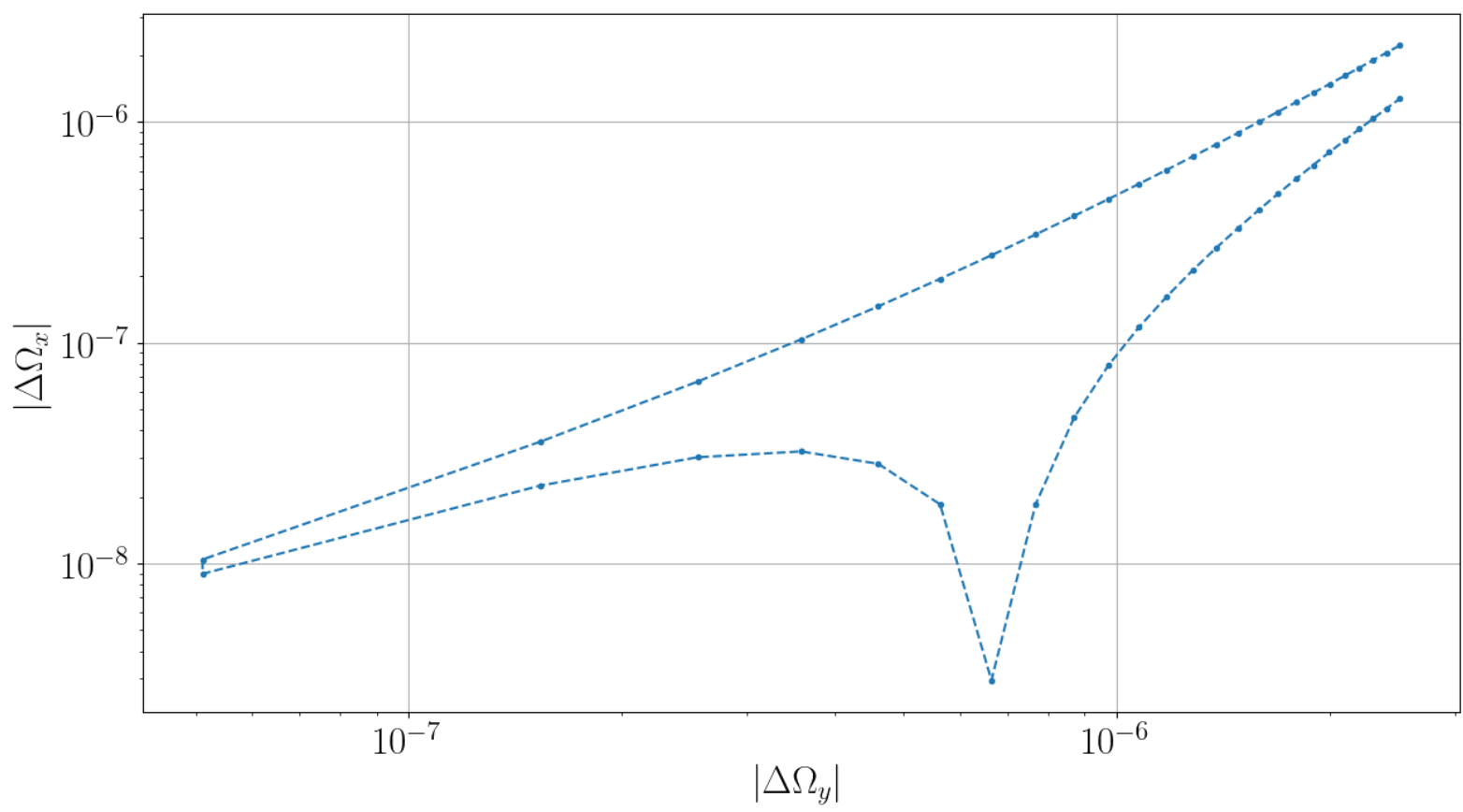

(b) Difference between the CW \& CCW beams' radial spin precession angular velocity components as a function of the differnce between their vertical components (calibration plot).

Figure 2.21: Simulation results in the case fo vertical plane synchrotron oscillations-related spin decoherence. 
CHAPTER 2. UNIVERSAL SR EDM MEASUREMENT PROBLEMS AND THEIR SOLUTIONS
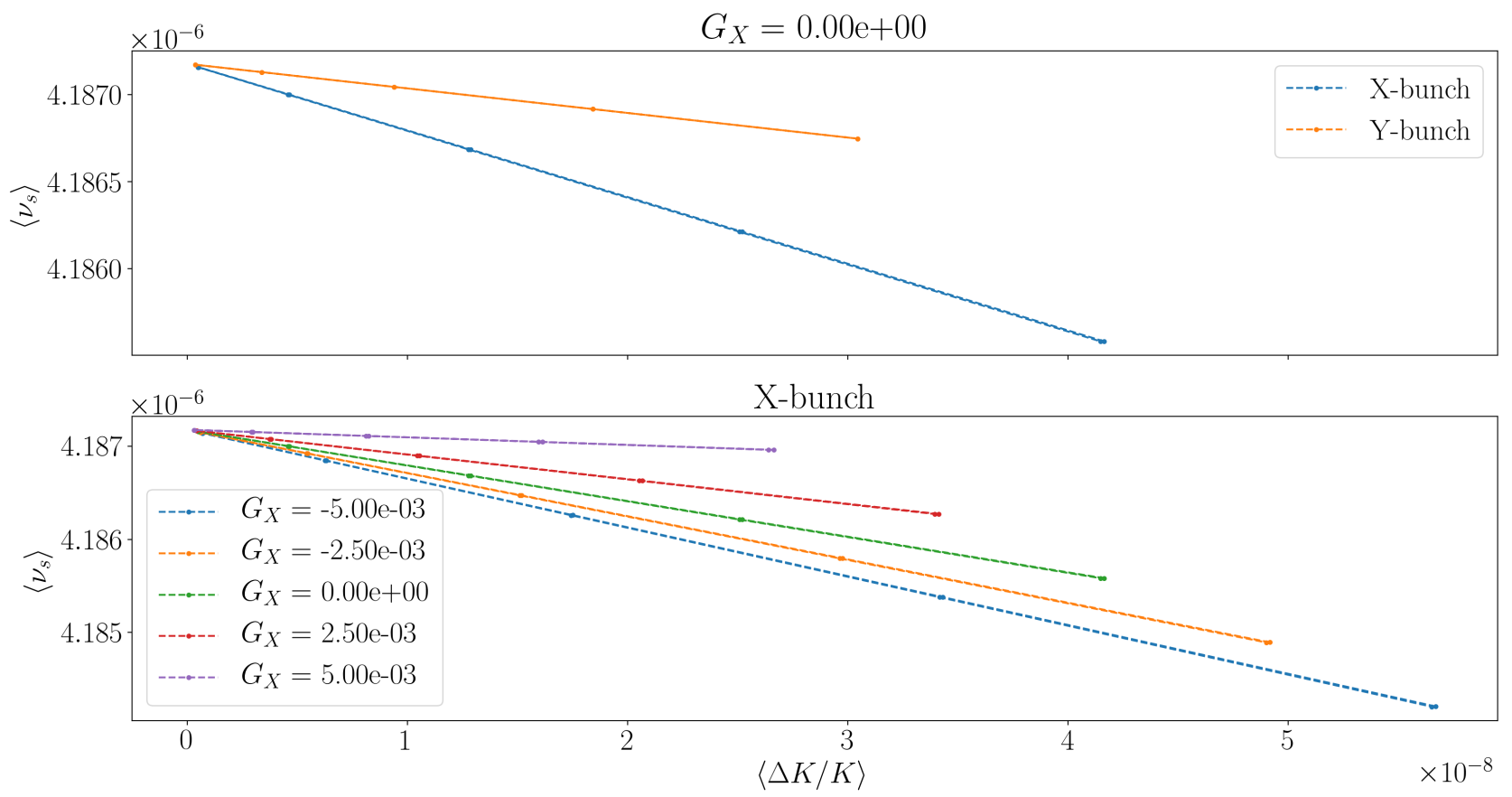

Figure 2.22: Particle mean spin tune level as a function of its mean kinetic energy level. Top panel: sextupoles are off for both injected bunches. Bottom panel: X-bunch dependencies at different GSX gradients. 
CHAPTER 2. UNIVERSAL SR EDM MEASUREMENT PROBLEMS AND THEIR SOLUTIONS
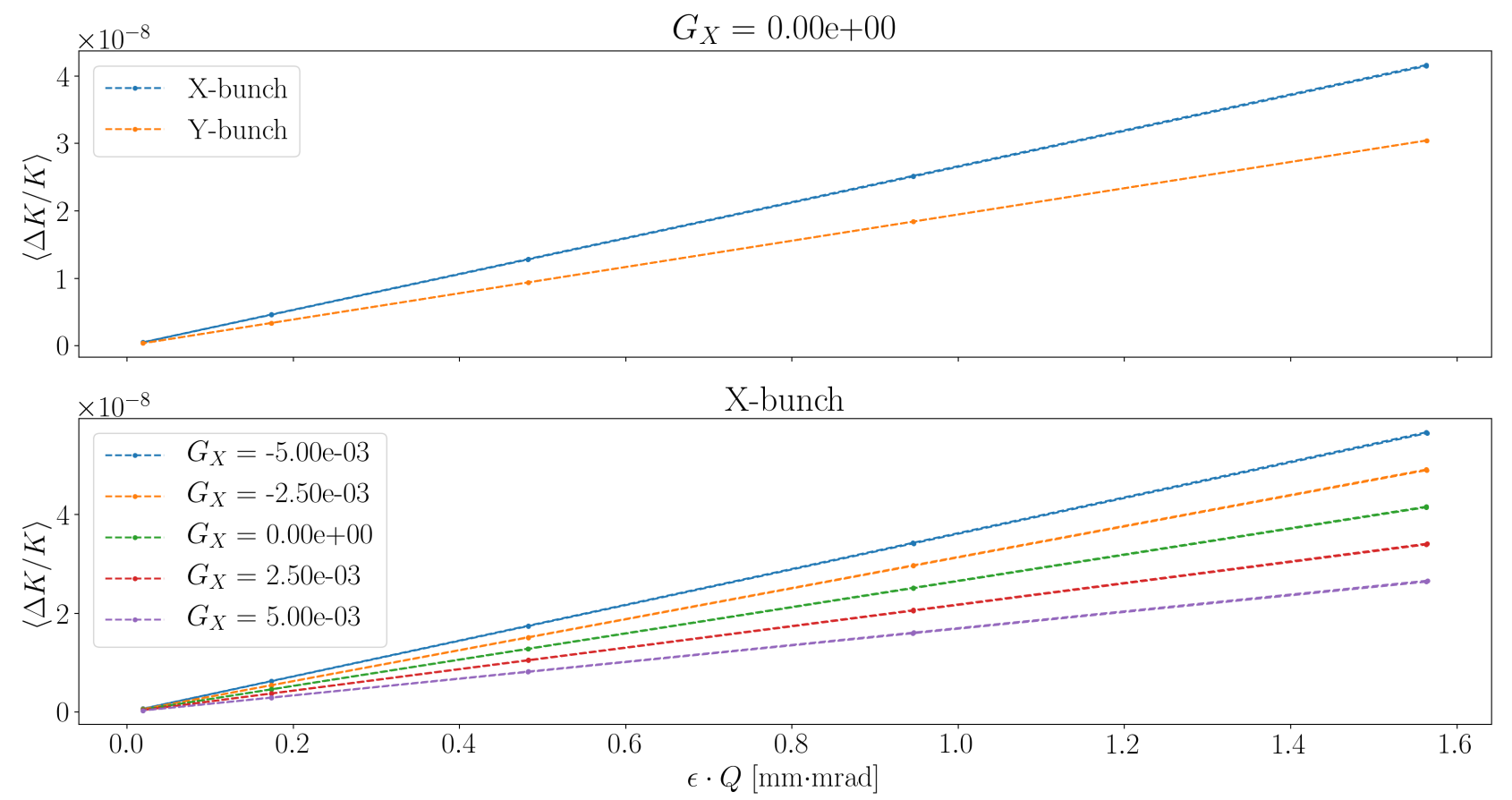

Figure 2.23: Longitudinal emittance dependence of the mean energy level. 
CHAPTER 2. UNIVERSAL SR EDM MEASUREMENT PROBLEMS AND THEIR SOLUTIONS
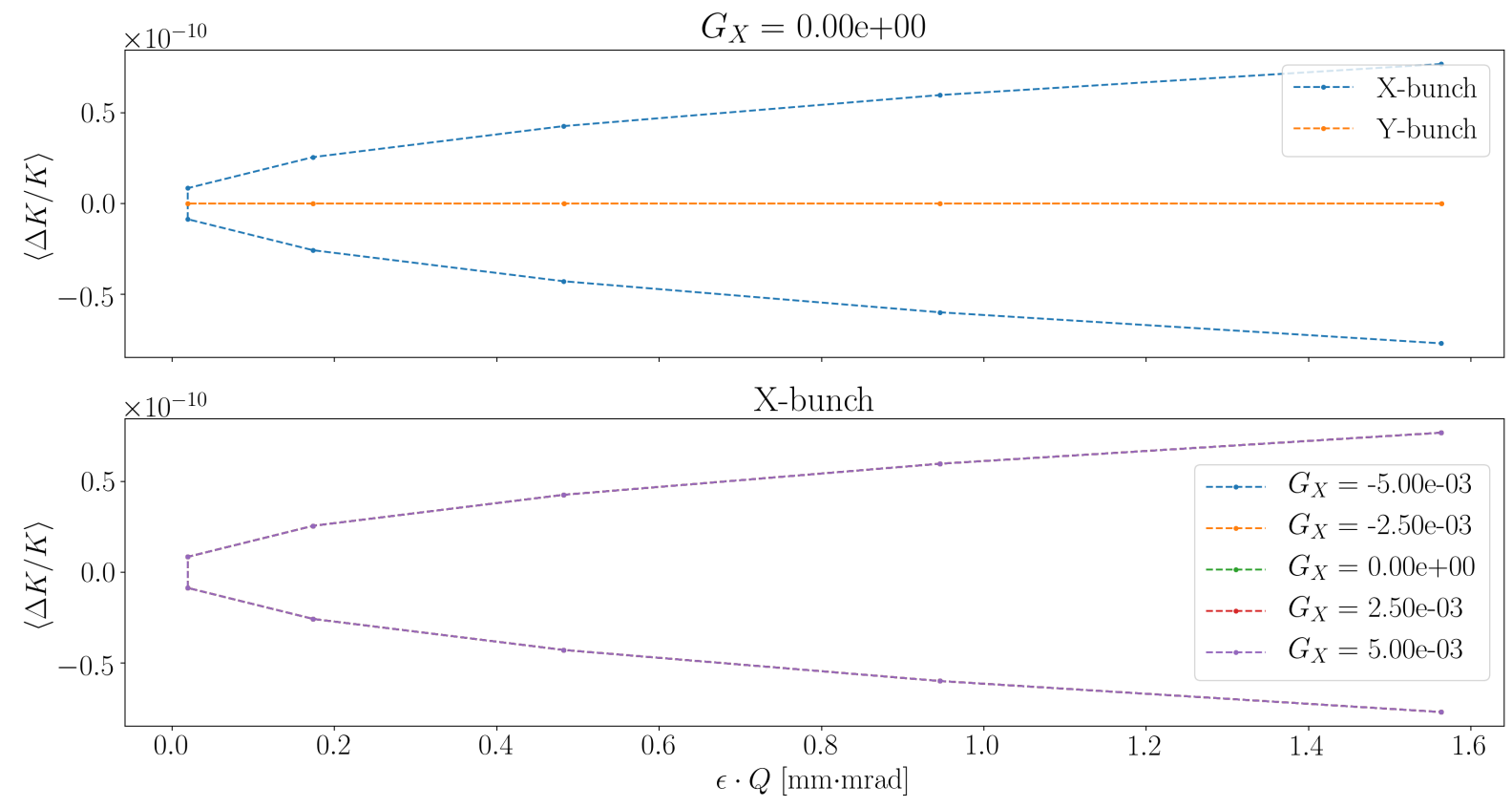

(a) Mean energy level dependence on particle transverse emittance
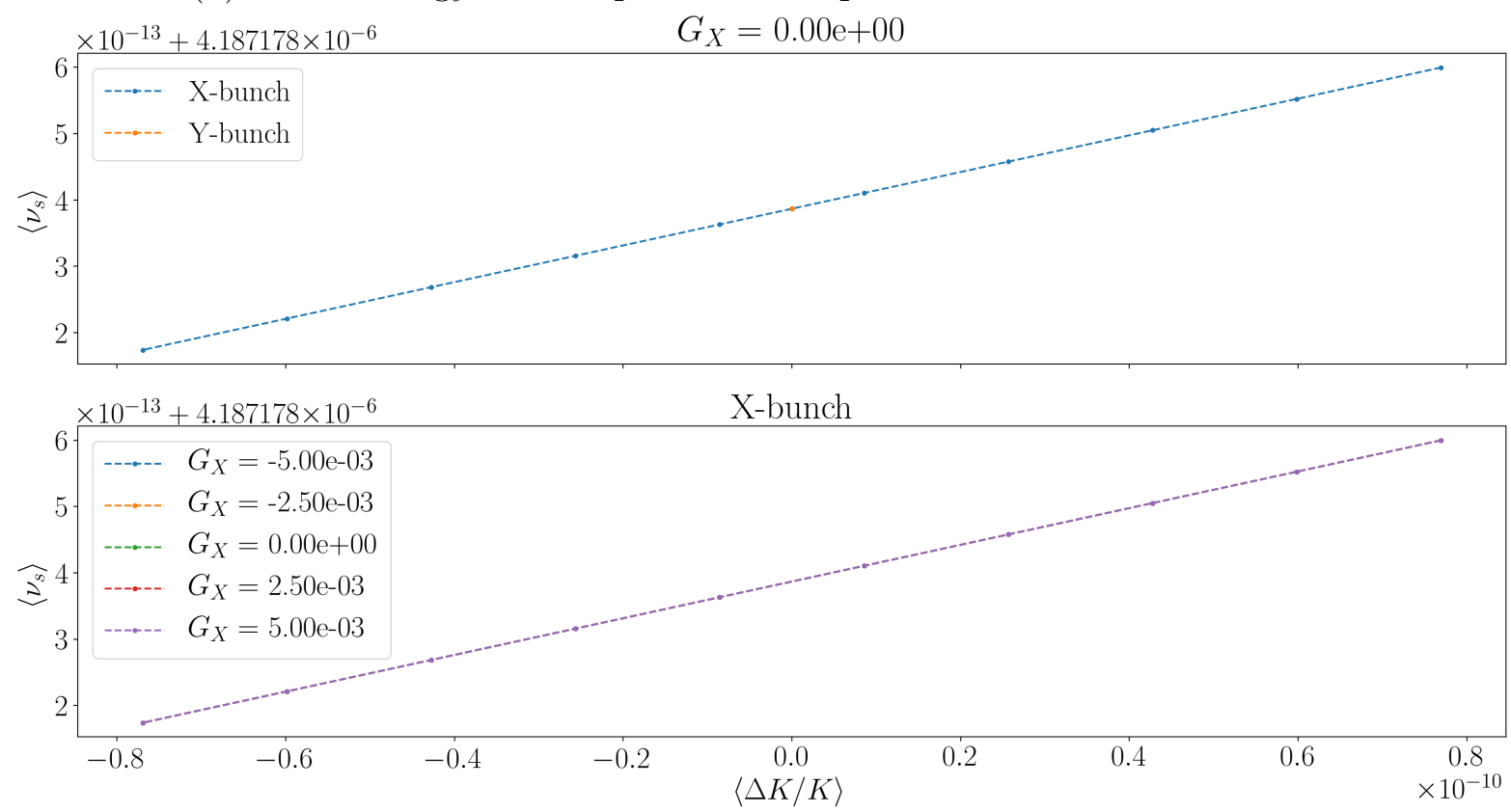

(b) Mean spin tune dependence on mean energy.

Figure 2.24: Simulation results in the case of linear transfer maps. 
CHAPTER 2. UNIVERSAL SR EDM MEASUREMENT PROBLEMS AND THEIR SOLUTIONS
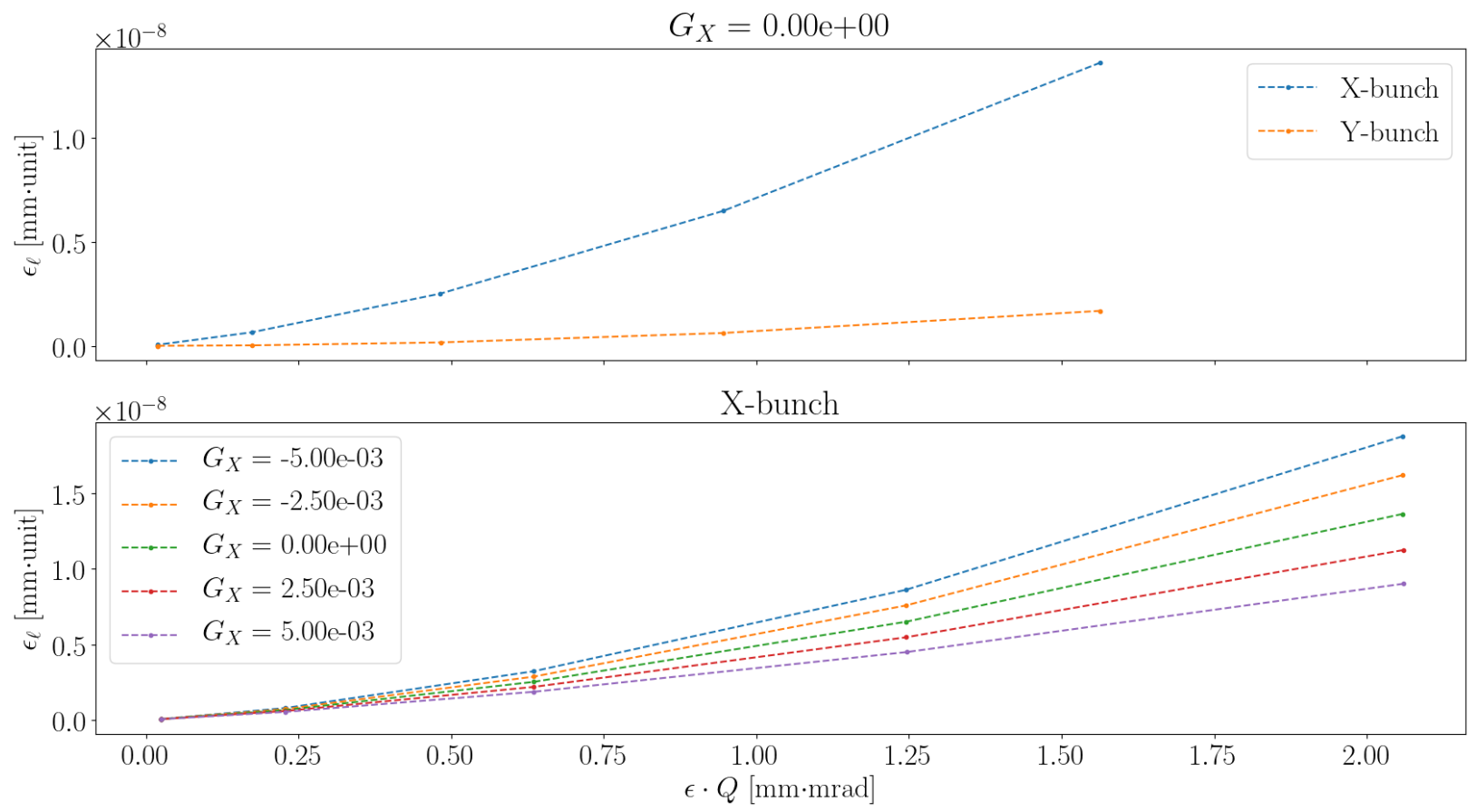

(a) Non-linear transfer maps.
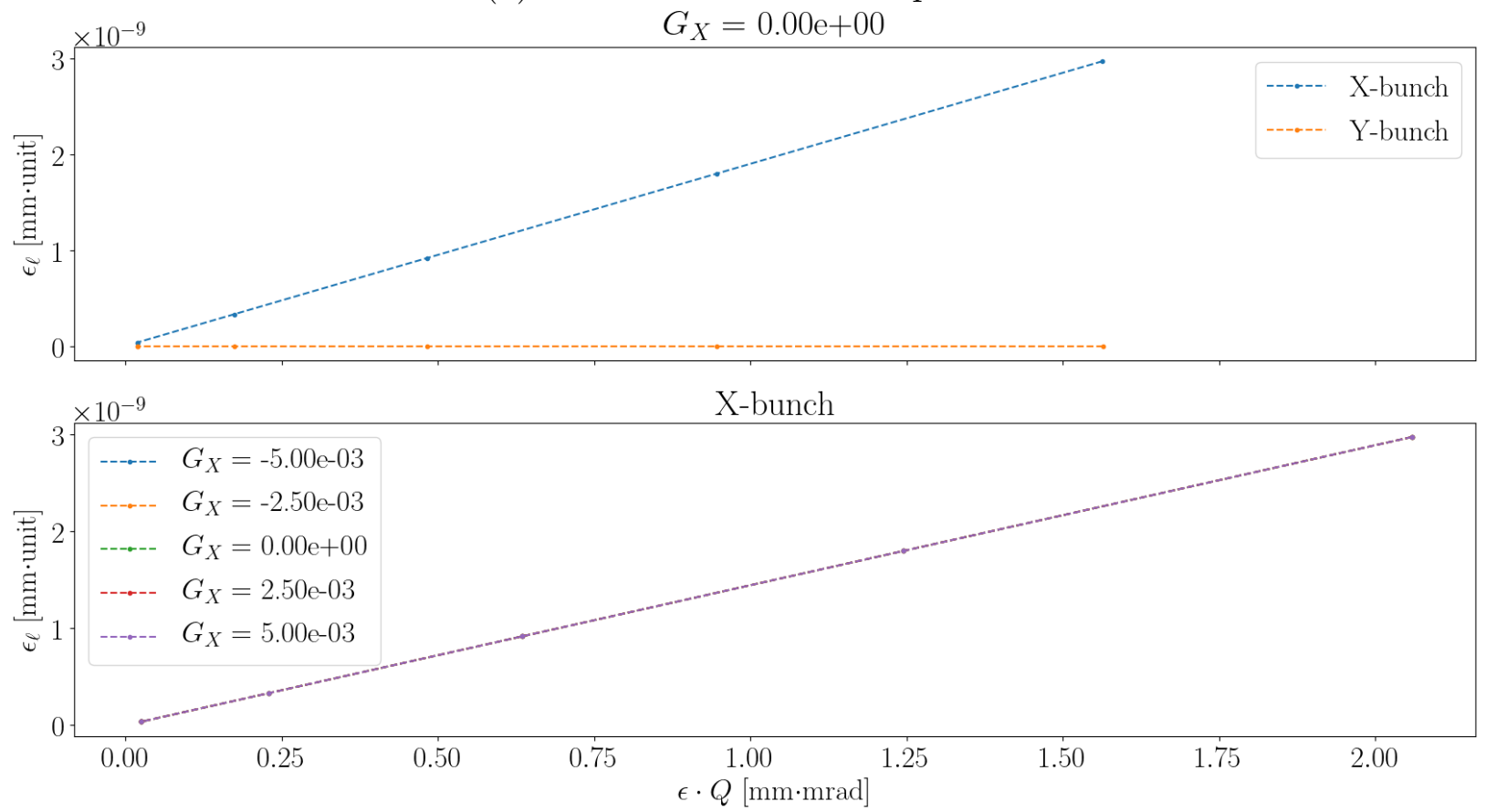

(b) Linear transfer maps.

Figure 2.25: Longitudinal emittance as a function of Q-normalized transverse emittance. 


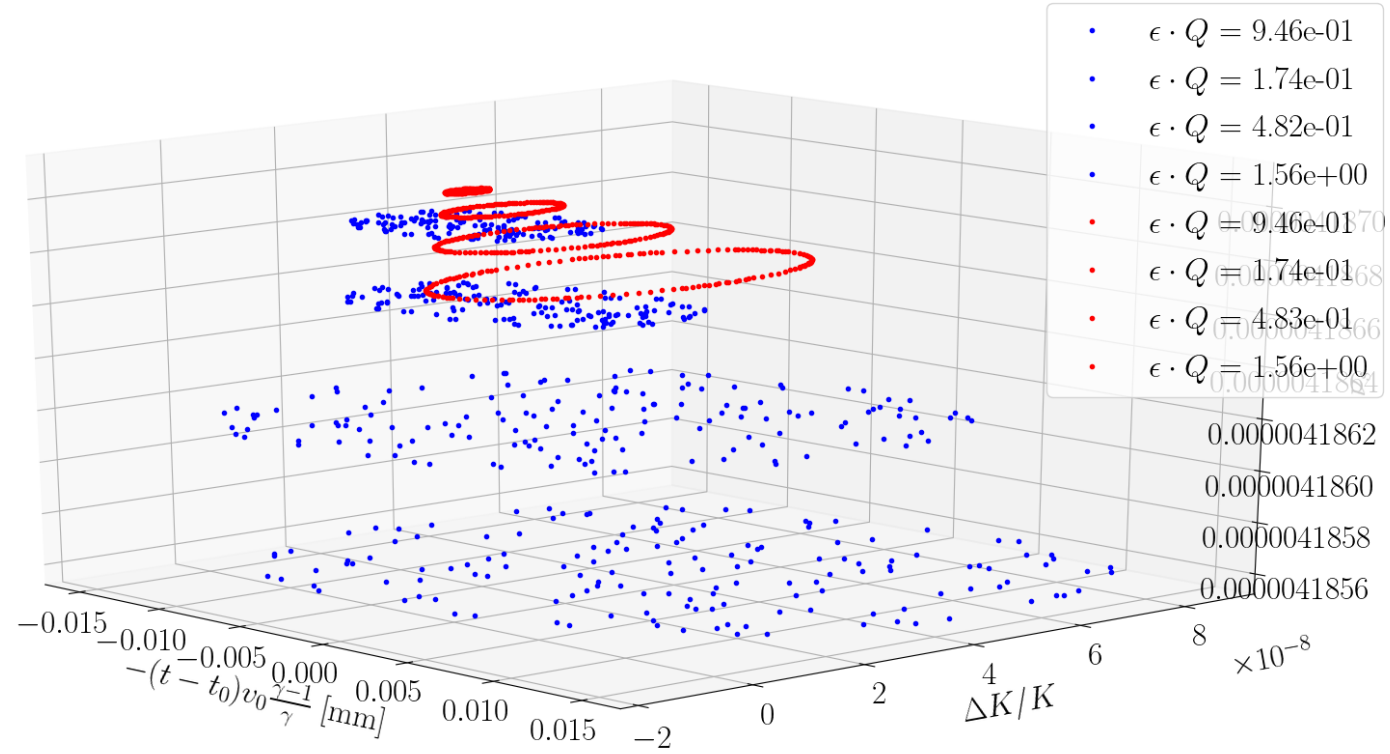

(a) Particles picked according to the values of their Q-normalized transverse emittances.

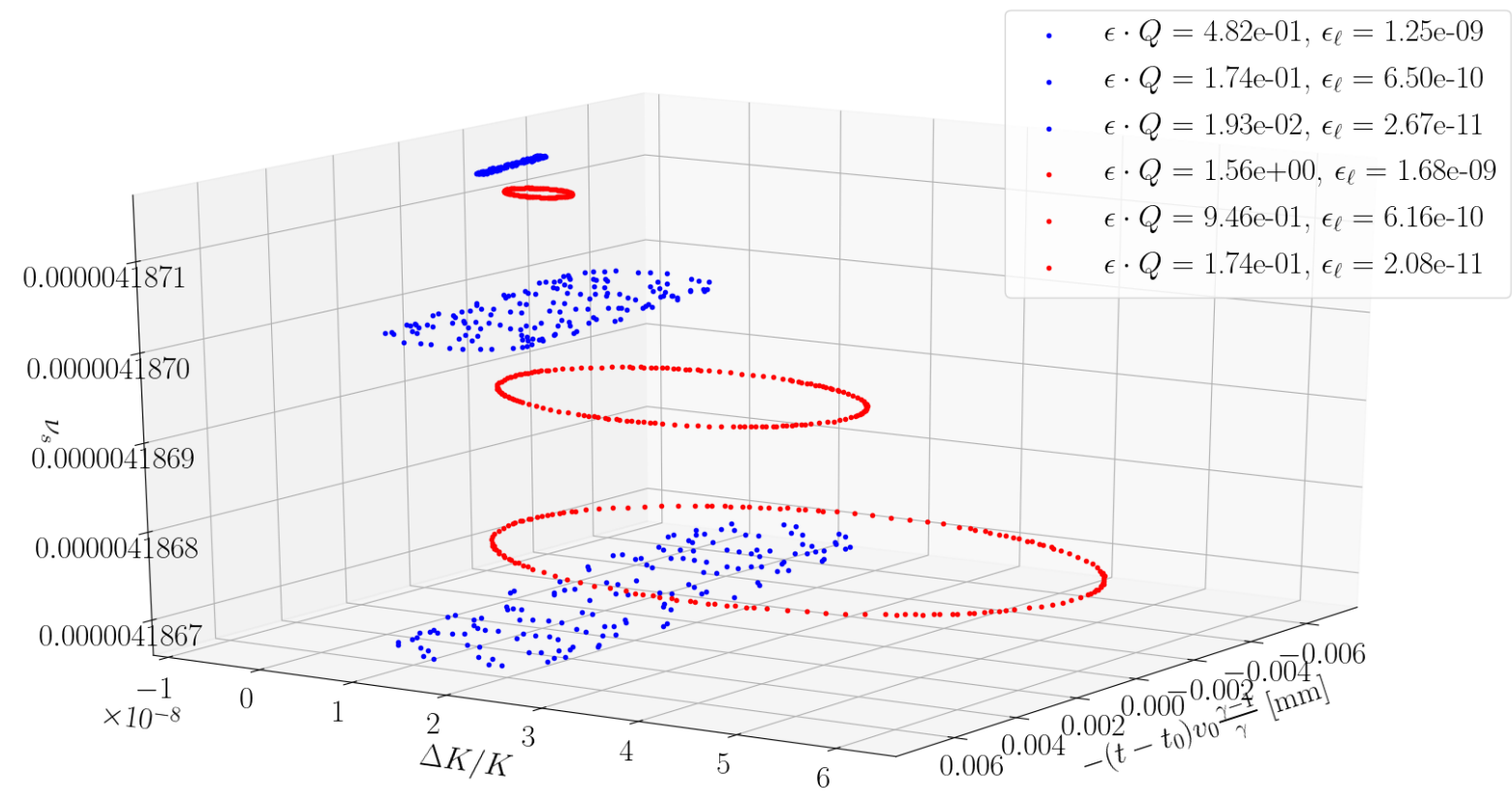

(b) Particles are picked according to the values of their longitudinal emittances.

Figure 2.26: Spin tune as a function of the particle's position in the longitudinal phase space. Colors mark the bunch: blue for X, red for Y. The corresponding Q-normalized transverse and longitudinal emittances are shown in the legend. 


\section{Chapter 3}

\section{Results at COSY}

\subsection{Synchrotron COSY}

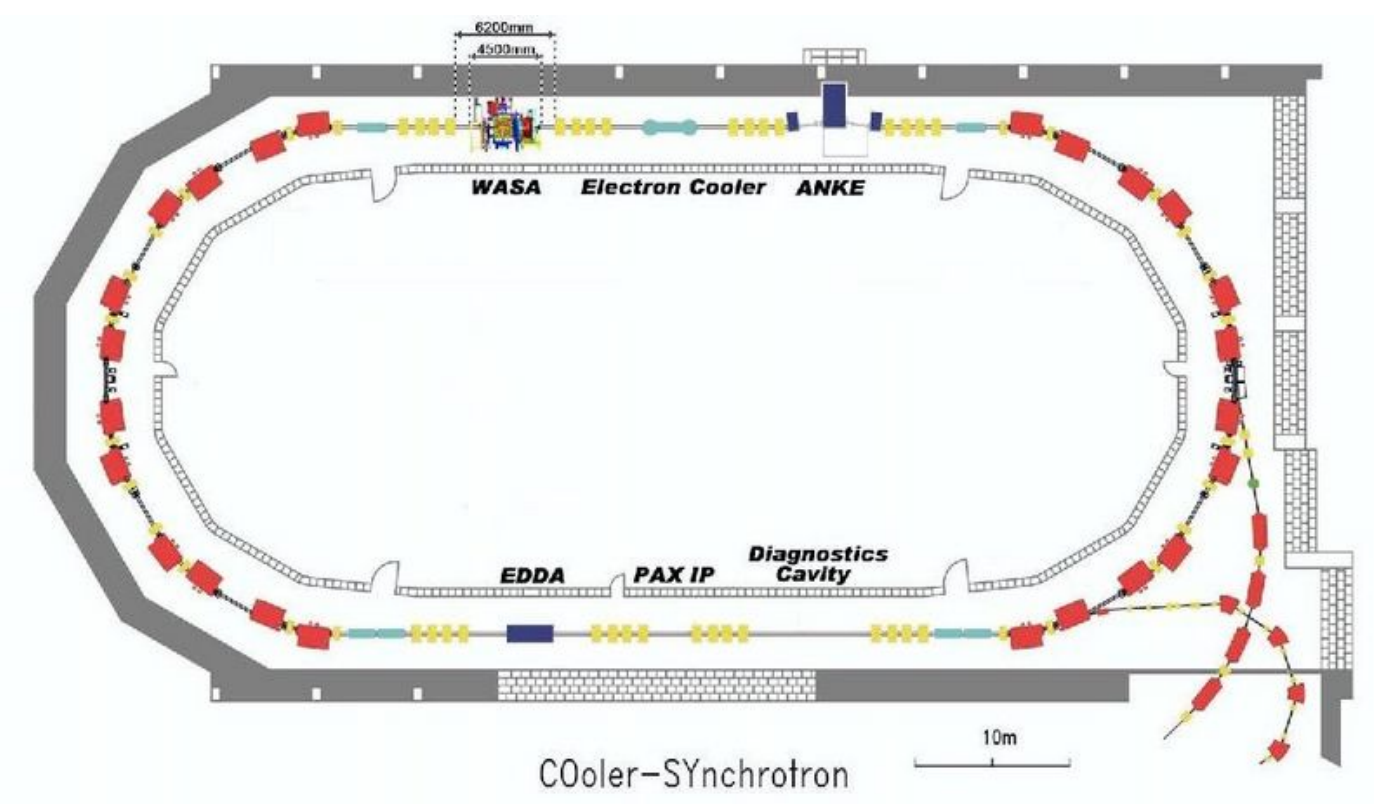

Figure 3.1: Synchrotron COSY.

The COSY accelerator facility depicted in Figure 3.1 consists of two sources of unpolarized $\mathrm{H}^{-} / \mathrm{D}^{-}$-ions and one source of polarized $\mathrm{H}^{-}$-ions, the injector cyclotron JULIC (Jülich Light Ion Cyclotron) [34] capable of accelerating the $\mathrm{H}^{-}$-ions up to $300 \mathrm{MeV} / \mathrm{c}$ and $\mathrm{D}^{-}$-ions up to $600 \mathrm{MeV} / \mathrm{c}$, 
and the cooler synchrotron ring COSY with a circumference $184 \mathrm{~m}$ accelerating protons and deuterons up to $3.3 \mathrm{GeV} / \mathrm{c}$. [35]

Injection into COSY is done via charge exchange of the negative ions over $20 \mathrm{~ms}$ with a linearly decreasing closed orbit bump at the position of the stripper foil. The polarized source delivers $10 \mu \mathrm{A}$ of polarized $\mathrm{H}^{-}$ions. [35]

Two types of beam cooling are available: electron (energy range in the "old" and "new" electron coolers: 20-100 keV and 20-2,000 keV, respectively) and stochastic. The two electron coolers installed in the straight sections are capable of cooling the beam in the full possible energy range. Stochastic cooling works in the momentum range $1.5-3.7 \mathrm{GeV} / \mathrm{c}$.

Beam polarization is continuously monitored by an internal polarimeter EDDA; recently, an additional polarimeter making use of WASA forward detectors was set up, and a new polarimeter, based on LYSO-scintillators, is under development and will be installed in the COSY ring in 2019. Proton polarization of $75 \%$ can be achieved up to the highest momentum levels; deuteron vector and tensor polarizations reach up to $60 \%$. [13, Historical background]

At present, a number of studies with polarized beams are carried out at COSY with the upshot of developing a future EDM experiment for an electrostatic storage ring. [36, 37, 15, 38, 39, 40] In most studies, parameters summarized in Table 3.1 are used.

Table 3.1: COSY parameters in most studies.

\begin{tabular}{lll}
\hline Parameter & Magnitude & Dimension \\
\hline COSY circumference & 184 & $\mathrm{~m}$ \\
Deuteron momentum & 970 & $\mathrm{MeV} / \mathrm{c}$ \\
$\beta / \gamma$ & $0.459 / 1.126$ & \\
Anomalous magnetic moment G & -0.143 & \\
Beam revolution frequency $f_{\text {rev }}$ & 752543 & $\mathrm{~Hz}$ \\
Measurement cycle length & $100-1500$ & $\mathrm{sec}$ \\
Number of particles in the bunch & $\approx 10^{9}$ & \\
\hline
\end{tabular}

The author would like to highlight the following technologies developed 
by the collaboration.

\subsection{High precision spin tune measurement}

The collaboration has been able to achieve an unprecedented spin tune measurement sensitivity level of $10^{-10}$.

The experiment consisted in the following. An initially verticallypolarized deuteron beam was injected into the ring. After the preparatory phase, during which the beam is cooled and bunched, the beam polarization was flipped into the horizontal plane by means of an RF solenoid inducing an imperfection resonance. [15, p. 7]

After that, the beam was continuously extracted onto a carbon target, and the up-down cross section asymmetry was measured, which is proportional the beam's horizontal polarization component. Due to the specially designed data acquisition system [41], it was possible to precisely determine the number of turns the beam had done in the ring by the time an event was recorded on the detector.

The main problem with this measurement was that spin tune could not be estimated via a regular model fit to polarimetry data. The spin precession frequency is approximately $120 \mathrm{kHz}$, while the detector sampling rate does not exceed $5 \mathrm{kHz}$, meaning that only one event could be detected per every 24 spin rotations about the vertical axis. To solve the problem of data sparsity all measurements were mapped into one oscillation period. [38]

This allowed for the estimation of the spin tune at a precision level of $10^{-10}$ in a 100 second measurement cycle, which theoretically allows for the detection of the EDM at the sensitivity level $10^{-24} e \cdot \mathrm{cm}$.

\subsection{Beam Based Alignment}

Beam Based Alignment [40] is a procedure to verify that the beam passes through the center of a quadrupole. In order to so do, one varies the strength of the quadrupole and observes the changes this affects in the 
closed orbit. If the beam does not pass through the center of the quadrupole, the closed orbit will shift; this shift can be described by

$$
\Delta x=\frac{\Delta k \cdot x\left(s_{0}\right) \ell}{B \rho} \cdot \frac{1}{1-k \frac{\ell \beta\left(s_{0}\right)}{2 B \rho \tan \pi \nu}} \cdot \frac{\sqrt{\beta(s) \beta\left(s_{0}\right)}}{2 \sin \pi \nu} \cos \left(\phi(s)-\phi\left(s_{0}\right)-\pi \nu\right),
$$

where $\Delta x$ is the orbit change; $s$ is the coordinate of the beam position monitor; $s_{0}$ is the coordinate of the quadrupole; $\Delta k$ is the quadrupole strength change; $\ell$ is the quadrupole length; $\nu$ is the betatron tune; $\phi$ is the betatron phase; $x\left(s_{0}\right)$ the beam position with respect to the magnetic center of the quadrupole.

Since the orbit change $\Delta x(s)$ is a linear function of the offset of the beam with respect to the magnetic center of the quadrupole, one can determine the optimal position of the quadrupole by minimizing the function

$$
f=\frac{1}{N_{\mathrm{BPM}}} \sum_{i=1}^{N_{\mathrm{BPM}}}\left(x_{i}(+\Delta k)-x_{i}(-\Delta k)^{2} \propto x^{2}\left(s_{0}\right) .\right.
$$

The first time, Beam Based Alignment was tested in the NovemberDecember 2017 beam time. The methodology requires that the strength of a single quadrupole is varied at a time, else the observed effect will be a superposition of several closed orbit perturbations. Since quadrupoles at COSY are fed in groups of four, in order to vary the strength of a single quadrupole, additional coils on the poles of a quadrupole are used. In that case, the field of a quadrupole becomes a superposition of two quadrupole fields; however, this does not reflect on the methodology.

During the measurement multiple different bumps were introduced into the closed orbit at the quadrupole position. This leads to different magnitudes of the measured effect on the closed orbit. Thereby multiple points could be scanned in horizontal and vertical direction to find the optimal beam position inside the quadrupole. [40, p. 60]

The measurement was repeated in February 2019.

From a surveying procedure the quadrupole position is known to approximately $0.2 \mathrm{~mm}$. [13, Results and achievements at COSY] 
Especially relevant for the present research are the spin coherence time optimization studies.

\subsection{Spin coherence time optimization procedure}

The initial goal of the spin coherence time (SCT) studies at COSY was to confirm the possibility of using sextupole fields in the correction of spin tune dispersion associated with the transverse beam emittances and momentum dispersion $(\Delta p / p)$. [42] At the present moment, SCT optimization is the initial phase of any EDM-related investigation at COSY.

Sextupole spin decoherence suppression is used together with electron cooling, in order to minimize the beam's phase space volume, and bunching, which is used for the suppression of the linear spin decoherence effect associated with momentum dispersion. The sextupoles, placed in the arc sections, are used for the suppression of second-order spin decoherence effects.

Spin decoherence is controlled by means of three sextupole families, marked respectively: MXG, placed in the maximum of the dispersion function, and controlling the decoherence associated with $\Delta p / p$; MXS, placed in the maximum of the horizontal beta-function $\beta_{x}$, and controlling the dispersion effect associated with horizontal betatron oscilaltions; MXL, in the maximum of $\beta_{y}$, controlling the dispersion assiciated with the vertical betatron oscillations.

\section{Optimization procedure}

In this section we describe the optimization procedure using the example of the 2014 experiment. [43] The SCT optimization experiment was first performed in 2012, but then only the MXS field strength was varied. In 2014, a comprehensive (the field strengths of all three sextupole families were varied) SCT optimization study was done for the first time. 


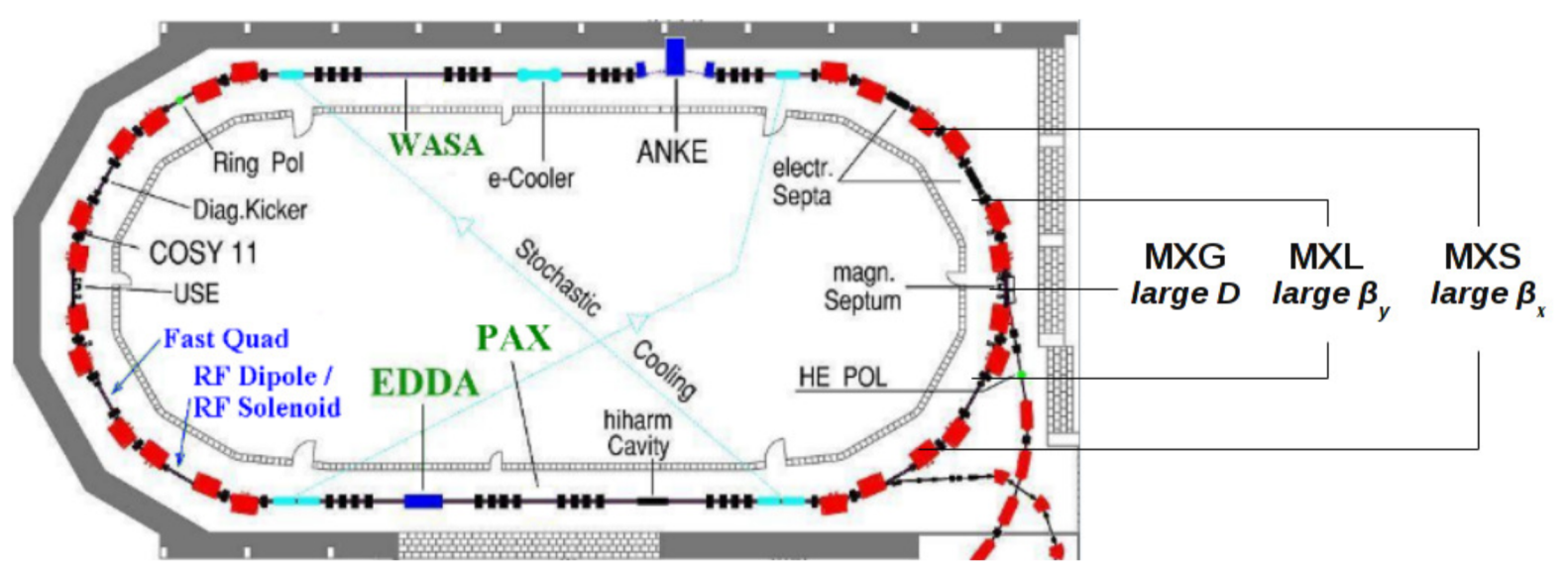

Figure 3.2: COSY ring with marked sextupole positions. (Image taken from [43].)

To separate the effects related to the beam emittances and the secondorder momentum dispersion $(\Delta p / p)^{2}$, the beam was prepared in two different ways.

When studying the effect associated with a large $(\Delta p / p)^{2}$, a polarized deuteron beam injected at $p=0.97 \mathrm{GeV} / \mathrm{c}$ momentum is first cooled for 60 seconds, so that its emittance is minimized. After the cooling is turned off, the beam is bunched (harmonic number $h=1$ ). Bunching is required to minimize linear spin decoherence effects.

When studying the effect associated with horizontal emittance, 1 the beam is cooled and bunched simultaneously for the initial 60 seconds, after which cooling is turned off, and horizontal heating is turned on for 5 seconds. The beam is heated by applying white noise to the horizontal kicker plates.

In both cases the polarization is vertically-oriented at injection. It is flipped by an RF solenoid after the beam preparation at the 80-th second.

Polarization is continuously measured by extracting the beam onto a $17 \mathrm{~mm}$ thick carbon target and detecting the scattered deuterons at the EDDA polarimeter. Extraction is done by applying white noise to the

\footnotetext{
${ }^{1}$ Decoherence associated with vertical emittance could not be studied because of acceptance limitations.
} 
vertical kicker. Elastic scattering of deuterons on carbon is a spin sensitive process with a large cross section.

The EDDA scintillators were grouped in four sectors (up, right, down, left); event rate asymmetry between the left and right sectors is proportional to the beam's vertical polarization, the one between the up and down - to the horizontal polarization component. Horizontal plane spin precession occurs at a a rate which greatly exceeds the polarimeter sampling rate, which is why a special data acquisition system was developed in 2012. [41]

As a result of the experiment [43] a possibility of reaching an SCT over 1,000 seconds at COSY was shown.

\section{SCT change when going from the external to the internal beam layers}

SCT optimization results obtained during the April-May 2019 beam time are shown below.

In the Figure series 3.3 the up-down cross section asymmetry measurement results are presented. In the first two figures one can observe that the rate of depolarization changes from high in the first half (100 to 150 seconds) of the measurement cycle to significantly lower in the second half. In Figure $3.3 \mathrm{a}$ especially, we observe that polarization is increasing in the 130 to 150 second range, before it begins to gradually decrease again.

Such behavior can be explained by the non-uniformity of the polarization distribution. In the first half of the measurement cycle particles from the outer (halo) beam layer are being sampled, while by the second half those get exhausted, and the polarization of the internal (core) layer is being probed. Since the core is more dense than the halo, the orbit length (hence spin tune) dispersion is less pronounced for its particles.

\section{SCT dependence on sextupole strength}

SCT dependence on the relative strengths of, respectively, the MXL and MXG sextupoles, measured during the April-May 2019 beamtime is pre- 
sented in Figure 3.4. A resonance-type pattern can be observed.

Since COSY operates at an energy that is significantly far removed from spin resonance we decided to check if this pattern can be seen (within the framework of our numerical model) in the FS-type lattice. Spin tune standard deviation as a function of the corresponding sextupole gradient is plotted in Figure 3.5. (Data were taken from the simulation described in section 2.2.) The same resonance pattern is observed as in the experiment. 
Horizontal Asymmetry FB Sum Pol=1 B0

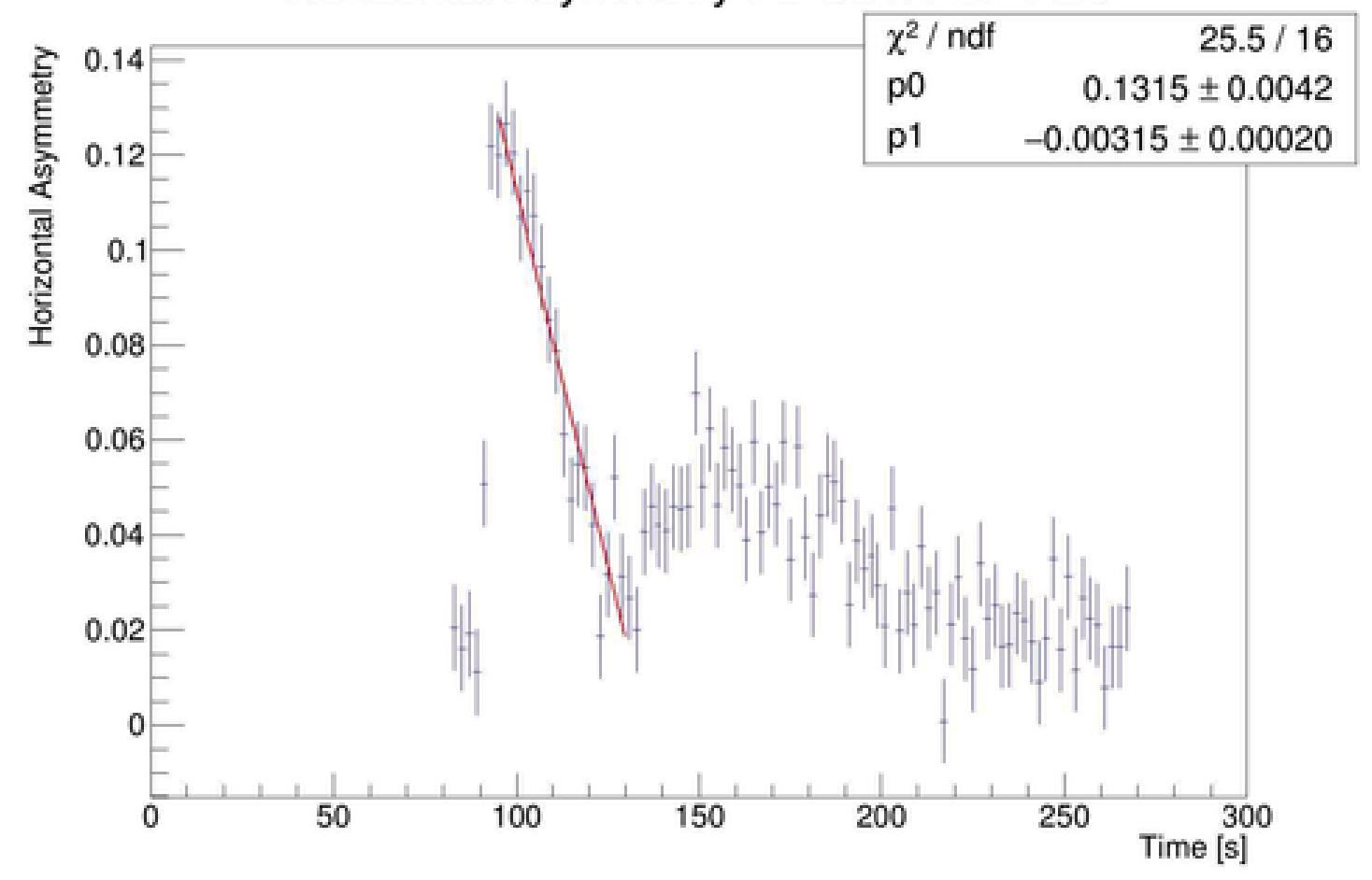

(a) $\mathrm{SCT}=20.87 \pm 1.49 \mathrm{sec}$.

Horizontal Asymmetry FB Sum Pol=1 B0

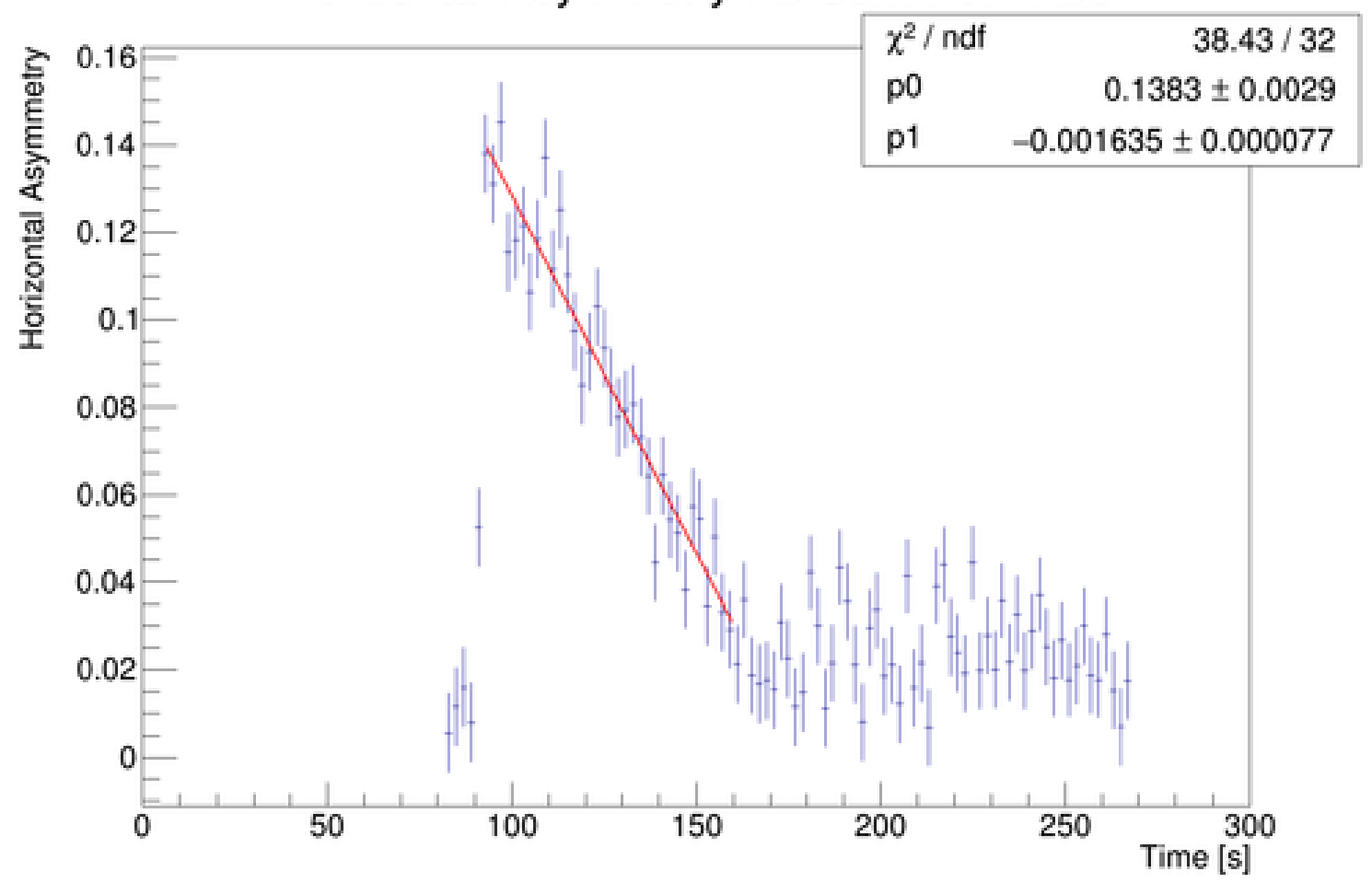

(b) $\mathrm{SCT}=42.3 \pm 2.2 \mathrm{sec}$. 
Horizontal Asymmetry FB Sum Pol=1 B0

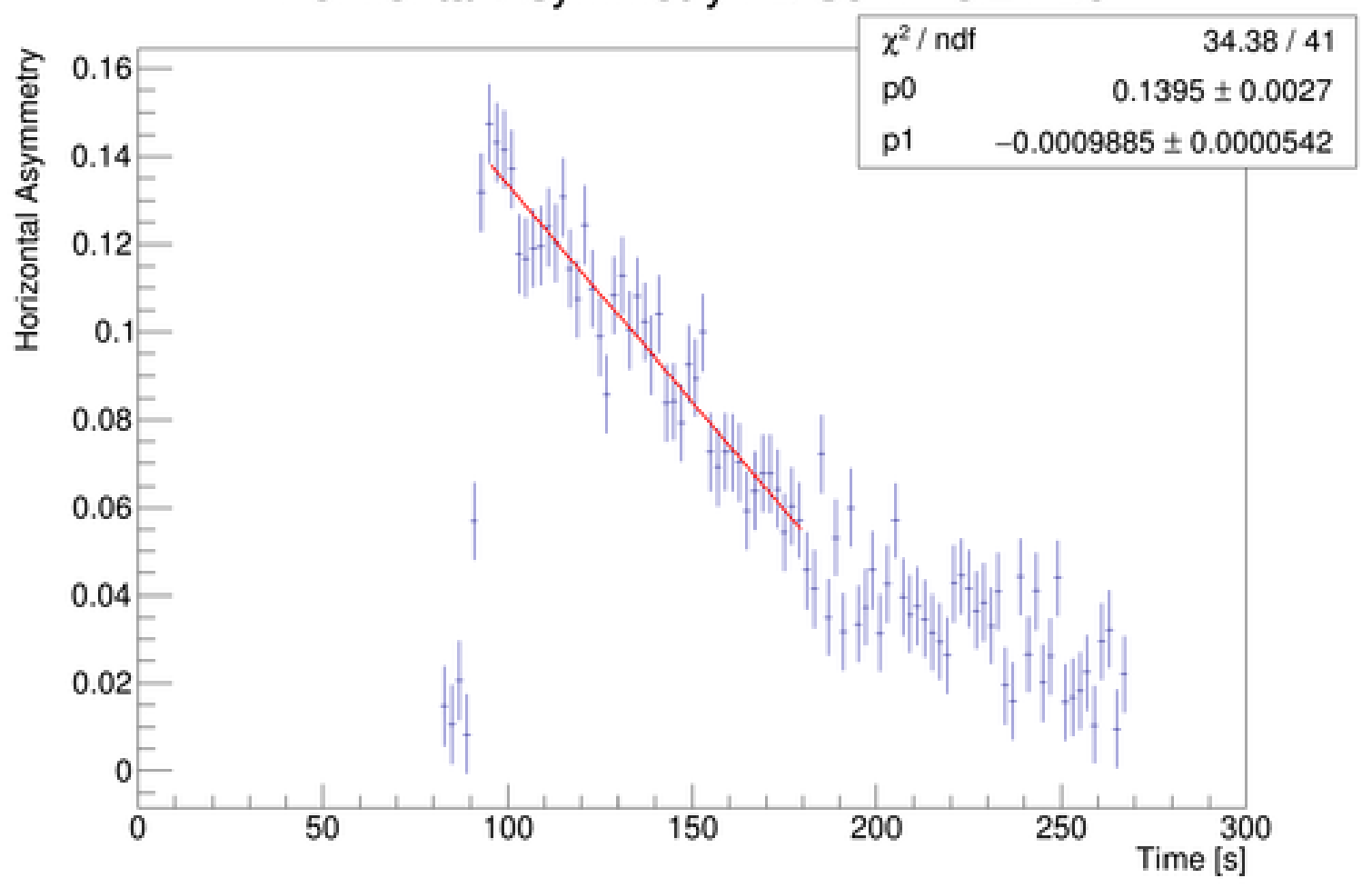

(c) $\mathrm{SCT}=70.6 \pm 4.1 \mathrm{sec}$.

Horizontal Asymmetry FB Sum Pol=1 B0

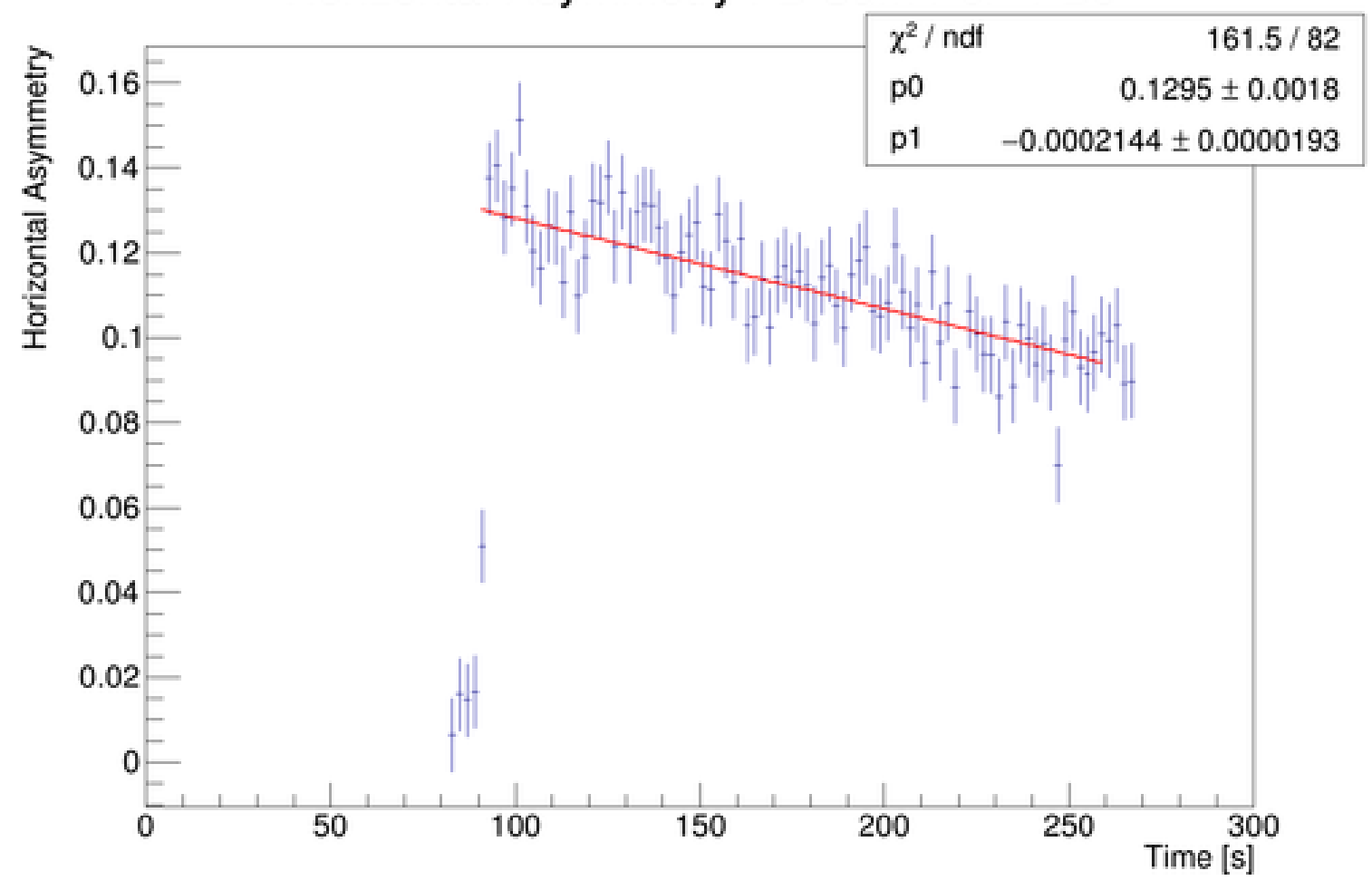

(d) $\mathrm{SCT}=302.0 \pm 27.5 \mathrm{sec}$.

Figure 3.3: Horizontal polarization measurements during SCT optimization during the axion search experiment done in April-May 2019. 
$M X L$ scan at $M X G=\%, M X S=\%$

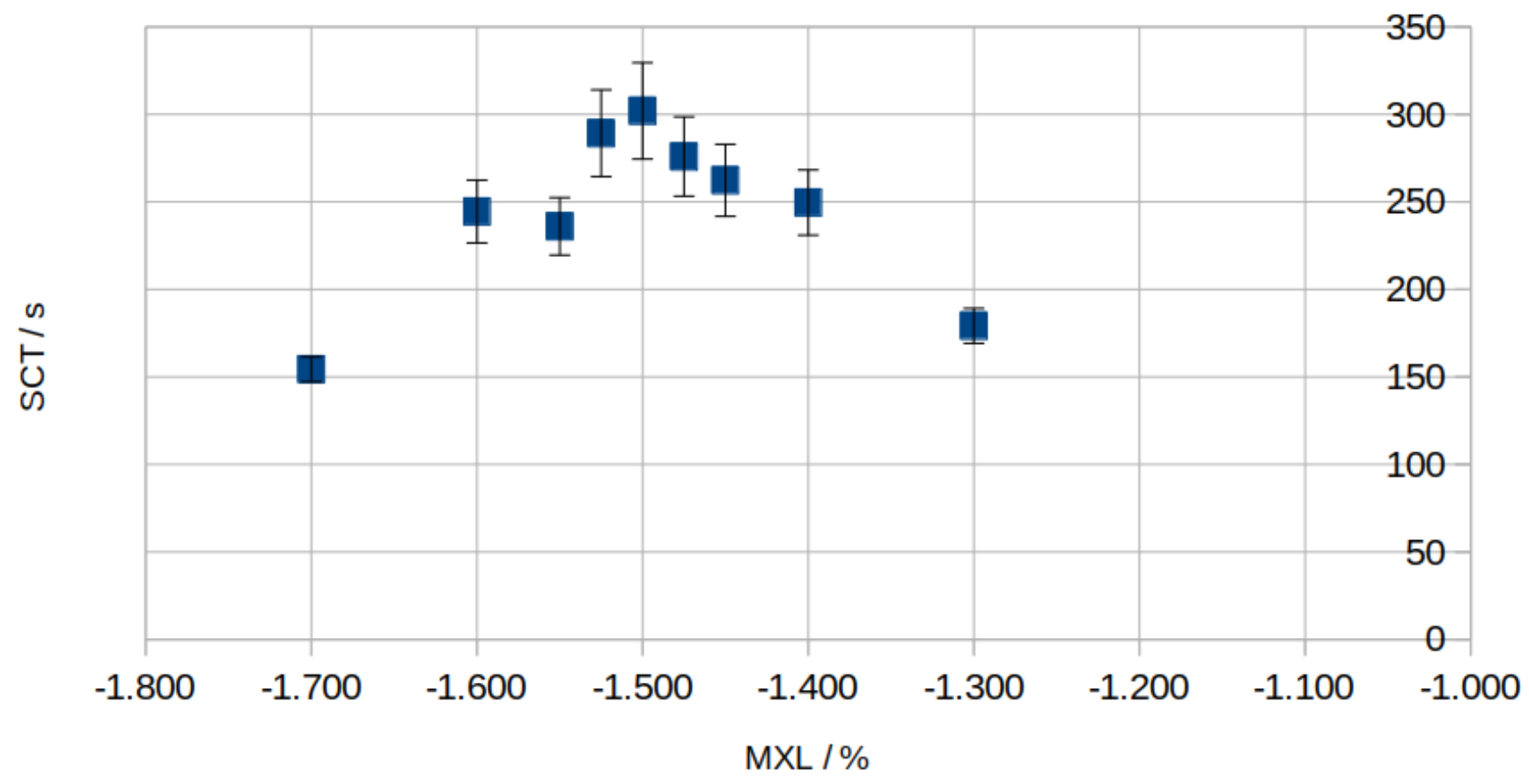

(a) MXL sextupole.

first diag. scan, $M X L-1.575 \%$

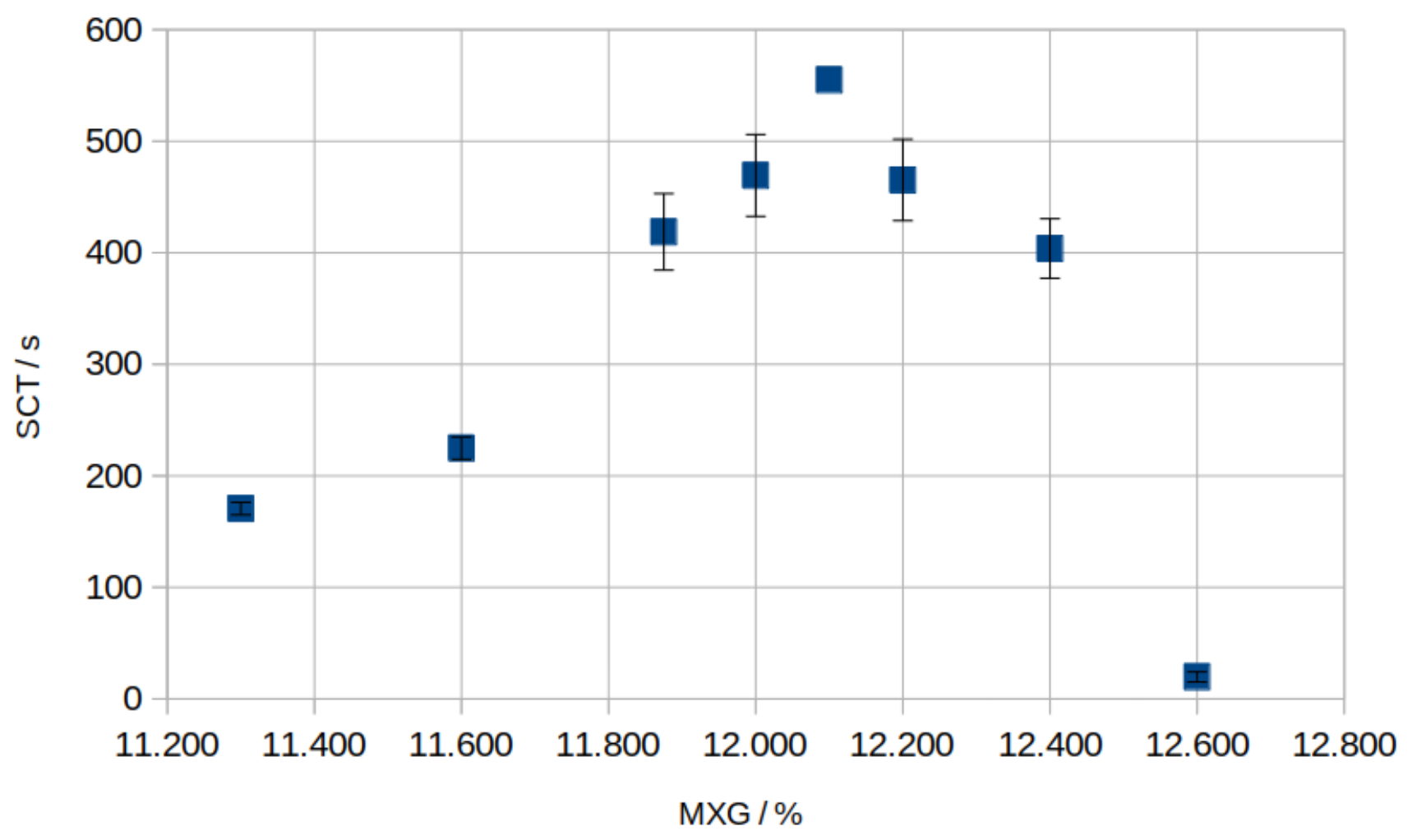

(b) MXG sextupole

Figure 3.4: SCT as a function fo the sextupole strength. 


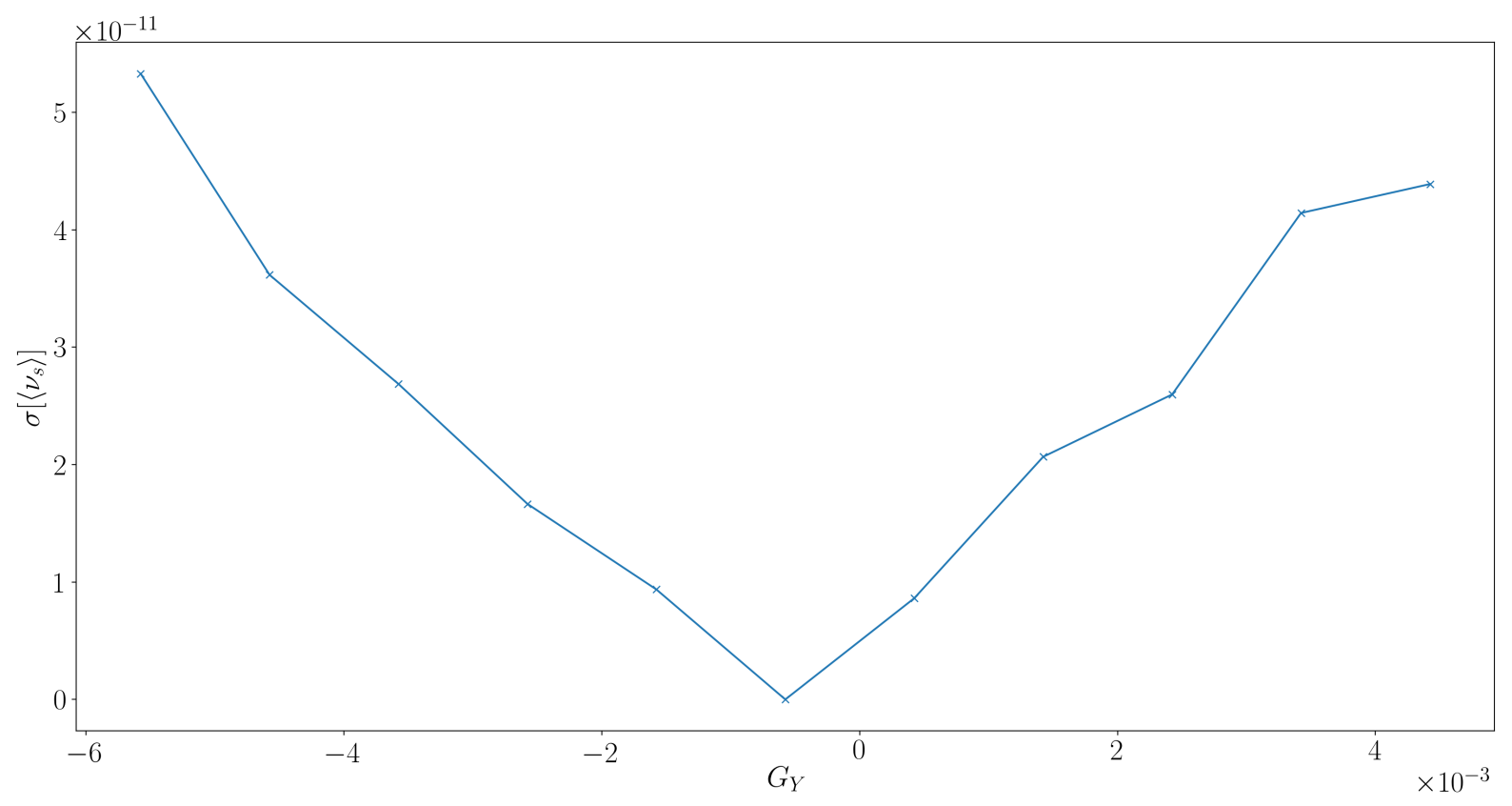

Figure 3.5: Spin tune dispersion as a function of the sextupole field gradient. 


\section{Conclusion}

The main results of this dissertation are:

(1) Effects of spin dynamics that could potentially result in systematic error were studiedm such as:

- betatron motion-related psrticle spin dynamics pertubrations;

- spin decoherence;

- machine imperfection-related EDM-faking MDM spin precession.

(2) For each of the systematic errors, a solution was described, its effectiveness numerically analyzed.

(3) Were formulated:

- the notions of the space and time domains (with respect to the FS SR EDM measurement methodology);

- the notion of the 2D frozen spin state;

- the necessary conditions of a successful SR EDM measurement;

- the 2D FS (Frequency Domain) method, satisfying all of the necessary conditions we found.

(4) Frozen and Quasi-Frozen spin lattices were described.

Concluding, I would like to express gratitude to my thesis advisors Y. V. Senichev and S. M. Polozov for guidigng me in this research, fellow students A. V. Saleev and E. V. Valetov for fruitful discussions, Institute 
fü Kernphysik (IKP-2) of Forschungszentrum Jülich, and in particular members of the JEDI Collaboration, for giving me the opportunity to work on this project and develop as a researcher. 


\section{Appendix A}

\section{Statistical modeling}

In this appendix we analyze the standard error of the spin precession frequency in the storage ring experiment for searching for the deuteron EDM. The main body of the analysis begins in section A.2, section A.1 introduces some terms (like sample Fisher information, Fisher information of a point), but it can be omitted.

Spin precession frequency is determined via fitting a harmonic signal $f(t)=a \cdot \sin (\omega \cdot t+\delta)$ with constant parameters $(a, \omega, \delta)$ to polarimetry data. Polarimetry data are obtained by scattering the polarized beam on a carbon target. Two important aspects of polarimetry are: (1) decrease of the number of beam particles at each measurement, and (2) depolarization.

The first aspect motivates the search for a more optimal beam sampling strategy. In sampling the beam polarization, most informative (in terms of frequency) are measurements made during a rapid change in the signal(see section A.1 below). This was the basis of the idea to measure polarization only when its vertical component crosses zero (frequency-modulated sampling): this way sampling is done in the mose efficient manner, and the beam lifetime is extended.

We must note, however, that the detector analyzing power is maximal in at the peaks of the measured signal, and goes to zero in the nodes. This limits the opportunities of improvement of the sampling effectiveness by modulated sampling: the most useful (for us) measurement are least certain, while the least useful can be measured with most certainty.

It also affects the heteroskedasticity of the data: in our simulation we 
used a non-periodic measurement error growth model [24, p. 18], while the oscillations of the analyzing power introduce periodicity into the error.

Depolarization is another factor restraining the usefulness of extending the beam lifetime; it puts a much harder bound on the duration of the measurement cycle, and hence the standard error of the frequency estimate obtained from a single cycle.

In the next sections we will introduce a detector counting rate model, the notion of cross section asymmetry, and determine an adequate (in view of depolarization) length of the polarization measurement cycle. We will also simulate experimental data in order to assess the potential of the frequency-modulated polarization samplign frequency.

\section{A.1 Preliminary analysis}

The probability of observing the value $y_{i} \equiv y\left(t_{i}\right)$ when the expectation value is $\mu\left(t_{i}\right)$ and the error is Gaussian is

$$
\begin{aligned}
f\left(y_{i} \mid \boldsymbol{\theta}\right) & =\frac{1}{\sqrt{2 \pi \nu}} \exp \left(-\frac{1}{2} \frac{\left(y_{i}-\mu\left(t_{i}\right)\right)^{2}}{\nu}\right), \\
\boldsymbol{\theta} & =(\nu, \omega, \phi), \\
\mu\left(t_{i}\right) & =N_{0}\left(1+P \sin \left(\omega t_{i}+\phi\right)\right) .
\end{aligned}
$$

The likelihood of observing a set of observations $\mathbf{y}=\left(y_{1}, \ldots, y_{K}\right)$, under the i.i.d. assumption, is the product of propabilities taken as a function of the parameters:

$$
\mathcal{L}(\boldsymbol{\theta} \mid \mathbf{y})=\prod_{i} f\left(y_{i} \mid \boldsymbol{\theta}\right),
$$

and the log-likelihood

$$
\ell(\boldsymbol{\theta} \mid \mathbf{y})=-\frac{K}{2} \log 2 \pi-\frac{K}{2} \log \nu-\frac{1}{2 \nu} \sum_{i} \epsilon_{i}^{2}, \epsilon_{i}=y_{i}-\mu\left(t_{i}\right) .
$$

The usual assumptions for the error term are zero expectation and strict exogeneity

$$
\mathrm{E}\left[\epsilon_{i} \mid \boldsymbol{\theta}_{0}\right]=\mathrm{E}\left[t_{i} \epsilon_{i} \mid \boldsymbol{\theta}_{0}\right]=0
$$


and the relations between the mean's derivatives are

$$
\begin{aligned}
& \mu_{\phi}^{\prime}=N_{0} P \cos (\omega t+\phi), \\
& \mu_{\omega}^{\prime}=t \cdot \mu_{\phi}^{\prime}, \epsilon_{\xi}^{\prime}=-\mu_{\xi}^{\prime} .
\end{aligned}
$$

\section{Variance of the frequency estimate}

After computing the lok-likelihood derivatives (and their expectation values), we can construct the Fisher matrix

$$
I\left(\boldsymbol{\theta}_{0}\right)=\left(\begin{array}{ccc}
K / 2 \nu & 0 & 0 \\
0 & 1 / \nu \sum\left(t_{i} \mu_{\phi}^{\prime}\left(t_{i}\right)\right)^{2} & 1 / \nu \sum t_{i}\left(\mu_{\phi}^{\prime}\left(t_{i}\right)\right)^{2} \\
0 & 1 / \nu \sum t_{i}\left(\mu_{\phi}^{\prime}\left(t_{i}\right)\right)^{2} & 1 / \nu \sum\left(\mu_{\phi}^{\prime}\left(t_{i}\right)\right)^{2}
\end{array}\right) .
$$

Its determinant

$$
\left|I\left(\boldsymbol{\theta}_{0}\right)\right|=\frac{K}{2 \nu^{3}} \underbrace{\left(\sum\left(t_{i} \mu_{\phi}^{\prime}\left(t_{i}\right)\right)^{2} \sum\left(\mu_{\phi}^{\prime}\left(t_{i}\right)\right)^{2}-\left(\sum t_{i}\left(\mu_{\phi}^{\prime}\left(t_{i}\right)\right)^{2}\right)^{2}\right)}_{\Omega} .
$$

The variance-covariance matrix

$$
v \operatorname{cov}=\left(\begin{array}{ccc}
2 \nu / K & 0 & 0 \\
0 & \nu \frac{\sum\left(\mu_{\phi}^{\prime}\left(t_{i}\right)\right)^{2}}{\Omega} & \nu \frac{\sum t_{i}\left(\mu_{\phi}^{\prime}\left(t_{i}\right)\right)^{2}}{\Omega} \\
0 & \nu \frac{\sum t_{i}\left(\mu_{\phi}^{\prime}\left(t_{i}\right)\right)^{2}}{\Omega} & \nu \frac{\sum\left(t_{i} \mu_{\phi}^{\prime}\left(t_{i}\right)\right)^{2}}{\Omega}
\end{array}\right) .
$$

The variance of the frequency estimate

$$
\operatorname{var}[\hat{\omega}]=\nu \frac{\sum\left(\mu_{\phi}^{\prime}\left(t_{i}\right)\right)^{2}}{\sum\left(t_{i} \mu_{\phi}^{\prime}\left(t_{i}\right)\right)^{2} \sum\left(\mu_{\phi}^{\prime}\left(t_{i}\right)\right)^{2}-\left(\sum t_{i}\left(\mu_{\phi}^{\prime}\left(t_{i}\right)\right)^{2}\right)^{2}} .
$$




\section{Cross-check}

Let $\mu\left(t_{i}\right)=\phi+\omega t_{i}$. In that case $\mu_{\phi}^{\prime}\left(t_{i}\right)=1, \mu_{\omega}^{\prime}\left(t_{i}\right)=t_{i}=t_{i} \cdot \mu_{\phi}^{\prime}\left(t_{i}\right)$, the determinant of the Fisher matrix simplifies to

$$
\begin{aligned}
\left|I\left(\boldsymbol{\theta}_{0}\right)\right| & =\frac{K}{2 \nu^{4}}\left(K \sum_{i} t_{i}^{2}-\left(\sum t_{i}\right)^{2}\right) \\
& =\frac{K^{3}}{2 \nu^{4}}\left(\frac{1}{K} \sum t_{i}^{2}-\langle t\rangle^{2}\right) \\
& =\frac{K}{2 \nu^{4}} \cdot \underbrace{K \sum\left(t_{i}-\langle t\rangle\right)^{2}}_{\Omega}
\end{aligned}
$$

and the variance-covariance matrix becomes

$$
v \operatorname{cov}=\left(\begin{array}{ccc}
2^{\nu^{2}} / K & 0 & 0 \\
0 & \frac{\nu}{\sum\left(t_{i}-\langle t\rangle\right)^{2}} & \nu \frac{\sum t_{i}}{K \sum\left(t_{i}-\langle t\rangle\right)^{2}} \\
0 & \nu \frac{\sum t_{i}}{K \sum\left(t_{i}-\langle t\rangle\right)^{2}} & \nu \frac{\sum t_{i}^{2}}{K \sum\left(t_{i}-\langle t\rangle\right)^{2}}
\end{array}\right),
$$

with the well-known expression for the slope variance

$$
\operatorname{var}[\hat{\omega}]=\frac{\nu}{\sum\left(t_{i}-\langle t\rangle\right)^{2}} .
$$

Let us denote $\left(\mu_{\phi}^{\prime}\left(t_{i}\right)\right)^{2}=\left(N_{0} P\right)^{2} \cos ^{2}\left(\omega t_{i}+\phi\right) \equiv x_{i}$. Eq. A.1 can be rewritten in the following form:

$$
\begin{aligned}
\operatorname{var}[\hat{\omega}] & =\frac{\nu}{\sum_{j} x_{j}\left(\sum_{i} t_{i}^{2} \frac{x_{i}}{\sum_{j} x_{j}}-\left(\sum_{i} t_{i} \frac{x_{i}}{\sum_{j} x_{j}}\right)^{2}\right)} \\
& =\frac{\nu}{\sum_{j} x_{j} \sum_{i} w_{i}\left(t_{i}-\langle t\rangle_{w}\right)^{2}} \\
& =\frac{\nu}{\sum_{j} x_{j} \cdot \operatorname{var}_{w}[t]} .
\end{aligned}
$$




\section{Sampling modulation}

Suppose we write the Fisher matrix as a sum:

$$
I\left(\boldsymbol{\theta}_{0}\right)=\sum_{i} I_{i}\left(\boldsymbol{\theta}_{0}\right) ; I_{i}\left(\boldsymbol{\theta}_{0}\right)=\frac{1}{\nu}\left(\begin{array}{ccc}
\left(\sqrt{2} \cdot \mu_{\phi}^{\prime}\left(t_{i}\right)\right)^{-2} & 0 & 0 \\
0 & t_{i}^{2} & t_{i} \\
0 & t_{i} & 1
\end{array}\right) \cdot\left(\mu_{\phi}^{\prime}\left(t_{i}\right)\right)^{2} .
$$

$I_{i}\left(\boldsymbol{\theta}_{0}\right)=-\mathrm{E}\left[\left.\frac{\partial^{2}}{\partial \boldsymbol{\theta}^{2}} \log f\left(y_{i} \mid \boldsymbol{\theta}\right)\right|_{\boldsymbol{\theta}=\boldsymbol{\theta}_{0}} \mid \boldsymbol{\theta}_{0}\right]$ could be $\mathrm{q}^{1}$ interpreted as the information about the parameter that's carried in $y_{i}$.

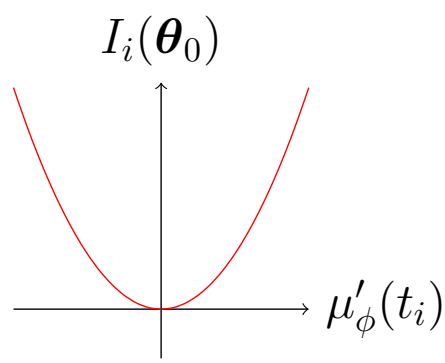

Figure A.1: Fisher information of a point is a parabola of the signal derivative.

If we attribute each point a weight proportional to its Fisher information, i.e. $w_{i}=\cos ^{2}\left(\omega t_{i}+\phi\right)$, $\left.\right|^{2}$ the weight of a region where $\left(\mu_{\phi}^{\prime}\left(t_{i}\right)\right)^{2} \geq 1 / 2$ is greater than that of an equivalent region with $\left(\mu_{\phi}^{\prime}\left(t_{i}\right)\right)^{2}<1 / 2$ by the factor:

$$
\int_{t_{0}}^{t_{1}} \cos ^{2}(\omega t+\phi) \mathrm{d} t=\frac{1}{\omega} \int_{\omega t_{0}}^{\omega t_{1}} \cos ^{2} \theta \mathrm{d} \theta=\frac{\Delta t}{2}+\frac{1}{2 \omega} \sin \omega \Delta t \cos \omega \Sigma t \approx 1.9 .
$$

\footnotetext{
${ }^{1}$ The $t_{i}$ in the structural matrix in eq. A.3 worries me, because it appears that a point carries more information simply by virtue of it being measured later in time; but as far as I can tell the reason for it is that it is assumed that the point labeled as $i$ is the $i$-th point in a series, and so a later point is more informative than a point closer to the origin, all other things being equal. And it's nothing new; in linear regression we also want our predictors to be as spread out as possible.

${ }^{2}$ The variance of $\omega$ is proportional to the $(2,2)$-minor, in which time doesn't figure, only the squared cosine.
} 


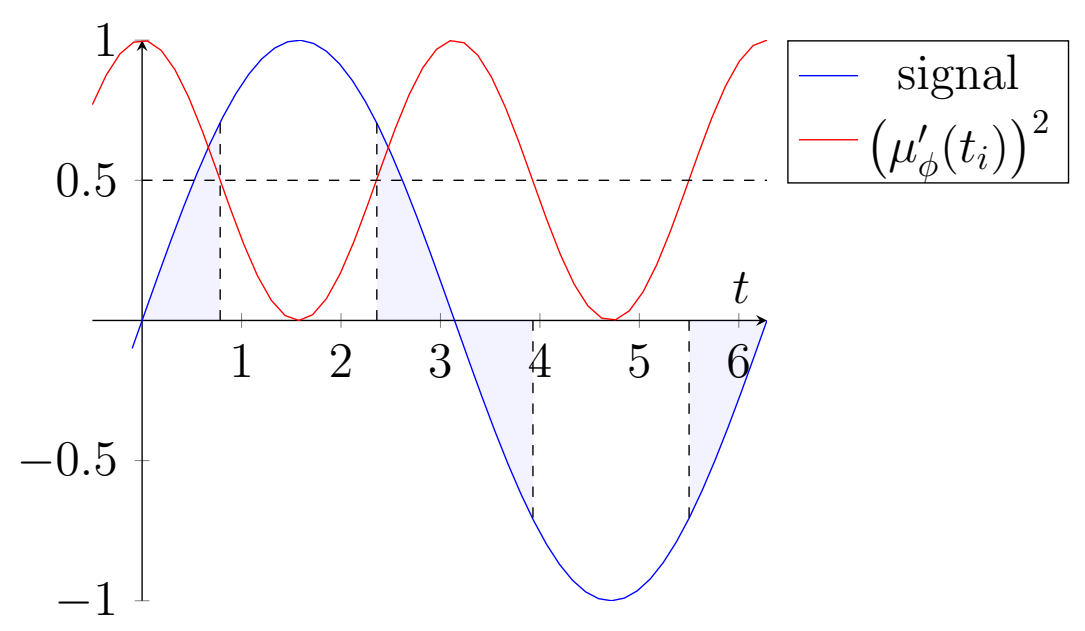

Figure A.2: Filled areas are where the points are more informative.

The implication is that increasing the number of points measured during the signal's rise and fall is roughly twice as beneficial as doing so during the peaks and troughs. $3^{3}$

\section{A.2 Detector counting rate model}

We assume the following model for the detector counting rate:

$$
N(t)=N_{0}(t) \cdot\left(1+P \cdot e^{-t / \tau_{d}} \cdot \sin (\omega \cdot t+\phi)\right),
$$

where $\tau_{d}$ is the decoherence lifetime, and $N_{0}(t)$ is the counting rate from the unpolarized cross-section.

Since the beam current can be expressed as a function of time as

$$
I(t) \equiv N^{b}(t) \nu=I_{0} \cdot e^{\lambda_{b} t}
$$

$\lambda_{b}$ the beam lifetime, the expected number of particles scattered in the ${ }^{3}$ This is not accounting for the fact that the certainty of a polarimetry measurement is
inversely proportional to its informativity, as was discussed in the introduction to this appendix. 
direction of the detector during measurement time $\Delta t_{c}$ is

$$
\begin{aligned}
N_{0}(t) & =p \cdot \int_{-\Delta t_{c} / 2}^{+\Delta t_{c} / 2} I(t+\tau) \mathrm{d} \tau \\
& =p \cdot \frac{\nu N_{0}^{b}}{\lambda_{b}} e^{\lambda_{b} t} \cdot\left(e^{\lambda_{b} \Delta t_{c} / 2}-e^{-\lambda_{b} \Delta t_{c} / 2}\right) \\
& \approx \underbrace{p \cdot \nu N_{0}^{b} e^{\lambda_{b} t}}_{\text {rate } r(t)} \cdot \Delta t_{c},
\end{aligned}
$$

where $p$ is the probability of "useful" scattering (approximately 1\%).

The actual number of detected particles will be distributed as a Poisson distribution

$$
P_{N_{0}(t)}\left(\tilde{N}_{0}\right)=\frac{\left(r(t) \Delta t_{c}\right)^{\tilde{N}_{0}}}{\tilde{N}_{0} !} \cdot e^{-r(t) \Delta t_{c}},
$$

hence $\sigma\left[\tilde{N}_{0}\right]^{2}(t)=N_{0}(t)$.

We are interested in the expectation value $N_{0}(t)=\mathrm{E}\left[\tilde{N}_{0}(t)\right]$, and its variance $\sigma\left[N_{0}\right](t)$. Those are estimated in the usual way, [?] as

$$
\left\langle\tilde{N}_{0}(t)\right\rangle_{\Delta t_{\epsilon}}=\frac{1}{n_{c / \epsilon}} \sum_{i=1}^{n_{c / \epsilon}} \tilde{N}_{0}\left(t_{i}\right), n_{c / \epsilon}=\Delta t_{\epsilon} / \Delta t_{c},
$$

and

$$
\sigma\left[\tilde{N}_{0}(t)\right]\left[\Delta t_{\epsilon}\right]=\frac{1}{n_{c / \epsilon}} \sum_{i=1}^{n_{c / \epsilon}}\left(\tilde{N}_{0}\left(t_{i}\right)-\left\langle\tilde{N}_{0}\left(t_{i}\right)\right\rangle_{\Delta t_{\epsilon}}\right)^{2} .
$$

( $\Delta t_{\epsilon}$ is the event measurement time, $\Delta t_{c}$ is the polarimetry measurement time.) A sum of random variables, $N_{0}(t)$ is normally distributed.

The standard error of the mean then is

$$
\begin{aligned}
\sigma\left[N_{0}\right](t) & =\sigma\left[\tilde{N}_{0}\right](t) / \sqrt{n_{c / \epsilon}}=\sqrt{N_{0}(t) \frac{\Delta t_{c}}{\Delta t_{\epsilon}}} \\
& \approx \sqrt{\frac{p \cdot \nu N_{0}^{b}}{\Delta t_{\epsilon}}} \cdot \Delta t_{c} \cdot \exp \left(\frac{\lambda_{b}}{2} \cdot t\right) .
\end{aligned}
$$


Relative error grows:

$$
\frac{\sigma\left[N_{0}\right](t)}{N_{0}(t)} \approx \frac{A}{\sqrt{\Delta t_{\epsilon}}} \cdot \exp \left(-\frac{\lambda_{b}}{2} t\right)=\frac{A}{\sqrt{\Delta t_{\epsilon}}} \cdot \exp \left(\frac{t}{2 \tau_{b}}\right), A=\frac{1}{\sqrt{p \cdot \nu N_{0}^{b}}} .
$$

\section{A.3 Cross section asymmetry}

A measure of the beam's polarization is the relative asymmetry of detector counting rates: [24, p. 17]

$$
\mathcal{A}=\frac{N\left(\frac{\pi}{2}\right)-N\left(-\frac{\pi}{2}\right)}{N\left(\frac{\pi}{2}\right)+N\left(-\frac{\pi}{2}\right)} .
$$

In the simulation to follow, the function fitted to the asymmetry data is:

$$
\mathcal{A}(t)=\mathcal{A}(0) \cdot e^{\lambda_{d} \cdot t} \cdot \sin (\omega \cdot t+\phi),
$$

with three nuisance parameters $\mathcal{A}(0), \lambda_{d}$, and $\phi$.

Due to the decreasing beam size, the measurement of the figure of merit is heteroscedastic. From [24, p. 18], the heteroscedasticity model assumed is

$$
\sigma[\mathcal{A}]^{2}(t) \approx \frac{1}{2 N_{0}(t)}
$$

\section{A.4 Measurement time frame}

Assuming a Gaussian error distribution with mean zero and variance $\sigma[\epsilon]^{2}$, the maximum likelihood estimator for the variance of the frequency estimate of the cross-section asymmetry $\mathcal{A}$ can be expressed as

$$
\operatorname{var}[\hat{\omega}]=\frac{\sigma[\epsilon]^{2}}{X_{t o t} \cdot \operatorname{var}_{w}[t]}
$$


with

$$
\begin{aligned}
X_{t o t} & =\sum_{j=1}^{n_{\epsilon}} x_{j}=\sum_{s=1}^{n_{z c}} \sum_{j=1}^{n_{\epsilon / z c}} x_{j s}, \\
\operatorname{var}_{w}[t] & =\sum_{i} w_{i}\left(t_{i}-\langle t\rangle_{w}\right)^{2},\langle t\rangle_{w}=\sum_{i} w_{i} t_{i}, \\
w_{i} & =\frac{x_{i}}{\sum_{j} x_{j}}, x_{i}=\left(\mathcal{A}(0) \exp \left(\lambda_{d} t_{i}\right)\right)^{2} \cos ^{2}\left(\omega t_{i}+\phi\right)=\left(\mu_{\phi}^{\prime}\left(t_{i}\right)\right)^{2} .
\end{aligned}
$$

In the expression above, $X_{\text {tot }}$ is the total Fisher information of the sample, and $\operatorname{var}_{w}[t]$ is a measure of its time-spread. It can be observed that by picking appropriate sampling times, one can raise the $X_{\text {tot }}$ term, since it is proportional to a sum of the signal's time derivatives. If the oscillation frequency and phase are already known to a reasonable precision, further improvement can be achieved by the application of a sampling scheme in which measurements are taken only during rapid change in the signal (sampling modulation). Improvement here is limited by the polarimetry sampling rate.

Both the $\operatorname{var}_{w}[t]$ and $X_{\text {tot }}$ terms are bounded as a result of spin tune decoherence. We can express $\sum_{j=1}^{n_{\epsilon} / z c} x_{j s}=n_{\epsilon / z c} \cdot x_{0 s}$, for some mean value $x_{0 s}$ at a given node $s . \quad n_{\epsilon / z c}$ is the number of asymmetry measurements per node. The period of time during which measuring takes place, $\Delta t_{z c}$, is termed compaction time. The value of the sum $\sum_{j=1}^{n_{\epsilon / z c}} x_{j s}$ falls exponentially due to decoherence, hence $x_{0 s}=x_{01} \exp \left(\lambda_{d} \cdot \frac{(s-1) \cdot \pi}{\omega}\right)$. Therefore,

$$
\begin{aligned}
X_{t o t} & =n_{\epsilon / z c} \cdot x_{01} \cdot \frac{\exp \left(\frac{\lambda_{d} \pi}{\omega} n_{z c}\right)-1}{\exp \left(\frac{\lambda_{d} \pi}{\omega}\right)-1} \equiv n_{\epsilon / z c} \cdot x_{01} \cdot g\left(n_{z c}\right) ; \\
x_{01} & =\frac{1}{\Delta t_{z c}} \int_{-\Delta t_{z c} / 2}^{+\Delta t_{z c} / 2} \cos ^{2}(\omega \cdot t) \mathrm{d} t=\frac{1}{2} \cdot\left(1+\frac{\sin \omega \Delta t_{z c}}{\omega \Delta t_{z c}}\right), \\
n_{\epsilon / z c} & =\frac{\Delta t_{z c}}{\Delta t_{\epsilon}} .
\end{aligned}
$$

Eq. A.11 provides us with a means to estimating the limits on the duration of the experiment. In Table A.1, the percentage of the total 
Fisher information limit, the time in decoherence lifetimes by which it is reached, and the signal-to-noise ratio by that time, are summarized. The signal-to-noise ratios are computed according to:

$$
\mathrm{SNR} \triangleq \frac{\mathcal{A}(0) \cdot e^{-t / \tau_{d}}}{\sigma[\mathcal{A}](t)} \approx \sqrt{2 \cdot p \cdot \nu N_{0}^{b} \cdot \Delta t_{c}} \cdot \mathcal{A}(0) \cdot \exp \left[-\frac{t}{\tau_{d}} \cdot\left(1+\frac{1}{2} \frac{\tau_{d}}{\tau_{b}}\right)\right]
$$

in which, from $\sigma[\mathcal{A}(0)] / \mathcal{A}(0) \approx 3 \%$, the factor before the exponent is 33 .

Table A.1: Total Fisher information, by what time it is reached, and the corresponding signal-to-noise ratio.

\begin{tabular}{rrr}
\hline FI limit $(\%)$ & Reached $\left(\times \tau_{d}\right)$ & SNR \\
\hline 95 & 3.0 & 0.4 \\
90 & 2.3 & 1.1 \\
70 & 1.2 & 5.5 \\
50 & 0.7 & 11.7 \\
\hline
\end{tabular}

Eq. A.10) can be rewritten in physical terms assuming zero-decoherence $\left(\lambda_{d}=0\right)$ and uniform sampling with sampling period $\Delta t$ :

$$
\begin{aligned}
X_{\text {tot }} & =\sum_{k=1}^{K} \mathcal{A}^{2}(0) \cos ^{2}\left(\omega t_{k}+\phi\right)=\frac{1}{2} \mathcal{A}^{2}(0) \cdot K, \\
\operatorname{var}_{w}[t] & =\sum_{k=1}^{K}\left(k \Delta t-\langle t\rangle_{w}\right)^{2} \underbrace{w_{k}}_{1 / K} \\
& \approx \frac{\Delta t^{2}}{12} K^{2}=\frac{T^{2}}{12},
\end{aligned}
$$

and so

$$
\operatorname{var}[\hat{\omega}]=\frac{24}{K T^{2}} \cdot\left(\frac{\sigma[\epsilon]}{\mathcal{A}(0)}\right)^{2} .
$$




\section{A.5 Simulation}

We simulated data from two detectors with parameters gathered in Table A.2 for $T_{t o t}=1000$ seconds, sampled uniformly at the rate $f_{s}=375$ $\mathrm{Hz}$. These figures are chosen for the following reason: the beam size in a fill is on the order of $10^{11}$ particles; if we want to keep the beam lifetime equal to the decoherence lifetime, we cannot exhaust more than $75 \%$ of it; only $1 \%$ of all scatterings are of the sort we need for polarimetry, so we're left with $7.5 \cdot 10^{8}$ useful scatterings. A measurement of the counting rate $N_{0}(t)$ with a precision of approximately $3 \%$ requires somewhere in the neighborhood of 2,000 detector counts, which further reduces the number of events to $3.75 \cdot 10^{5}=f_{s} \cdot T_{\text {tot }}$. One thousand seconds is the expected duration of a fill, hence $f_{s}=375 \mathrm{~Hz}$.

Relative measurement error for the detector counting rates is depicted in Figure A.3; the cross-section asymmetry, computed according to Eq. (A.7), is shown in Figure A.4. To these data we fit via Maximum Likelihood a non-linear heteroscedastic model $]^{4}$ given by Eq. (A.8), with the variance function for the weights given by Eq. A.9). The fit results are summarized in Table A.3.

Table A.2: Detector counting rates' model parameters

\begin{tabular}{|c|c|c|}
\hline & Left Right & \\
\hline$\phi$ & $-\pi / 2 \quad+\pi / 2$ & $\mathrm{rad}$ \\
\hline$\omega$ & 3 & $\mathrm{rad} / \mathrm{sec}$ \\
\hline$P$ & 0.4 & \\
\hline$\tau_{d}$ & 721 & $\mathrm{sec}$ \\
\hline$\tau_{b}$ & 721 & sec \\
\hline$N_{0}(0)$ & 6730 & \\
\hline
\end{tabular}

If our initial frequency estimate obtained from a time-uniform sample has a standard error on the order of $1 \cdot 10^{-6} \mathrm{rad} / \mathrm{sec}$, simulation shows the standard error of the estimate can be improved to $\approx 5.8 \cdot 10^{-7} \mathrm{rad} / \mathrm{sec}$.

\footnotetext{
${ }^{4} \mathrm{R}$ package nlreg. [?]
} 
Table A.3: Fit results

\begin{tabular}{crrc}
\hline & Estimate & SE & Unit \\
\hline $\mathcal{A}(0)$ & 0.400 & $9.03 \cdot 10^{-5}$ & \\
$\lambda_{d}$ & -0.001 & $7.86 \cdot 10^{-7}$ & $1 / \mathrm{sec}$ \\
$\omega$ & 3.000 & $7.55 \cdot 10^{-7}$ & $\mathrm{rad} / \mathrm{sec}$ \\
$\phi$ & -1.571 & $2.25 \cdot 10^{-2}$ & $\mathrm{rad}$ \\
\hline
\end{tabular}




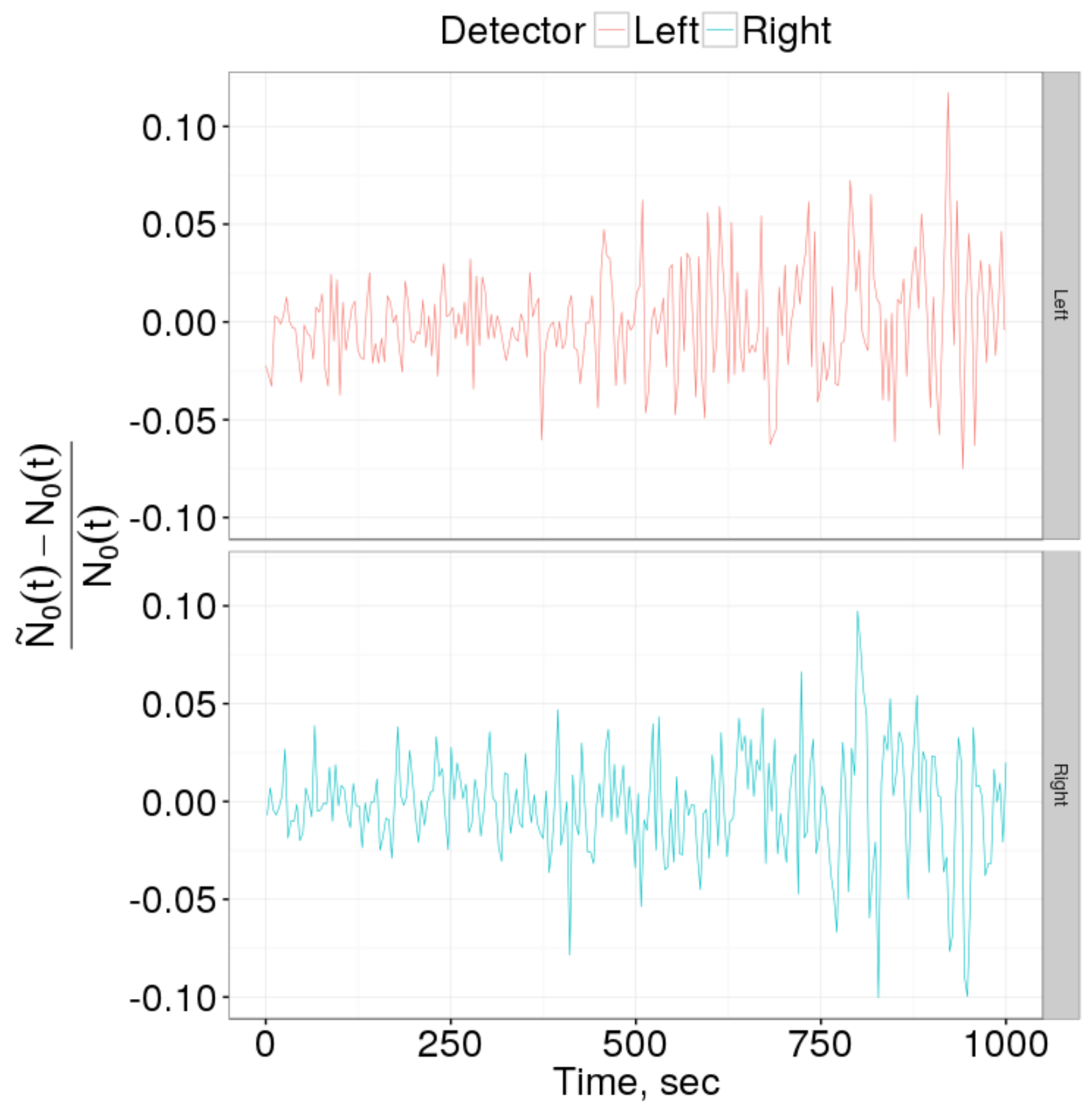

Figure A.3: Relative counting rate measurement error for the left and right detectors as a function of time. 


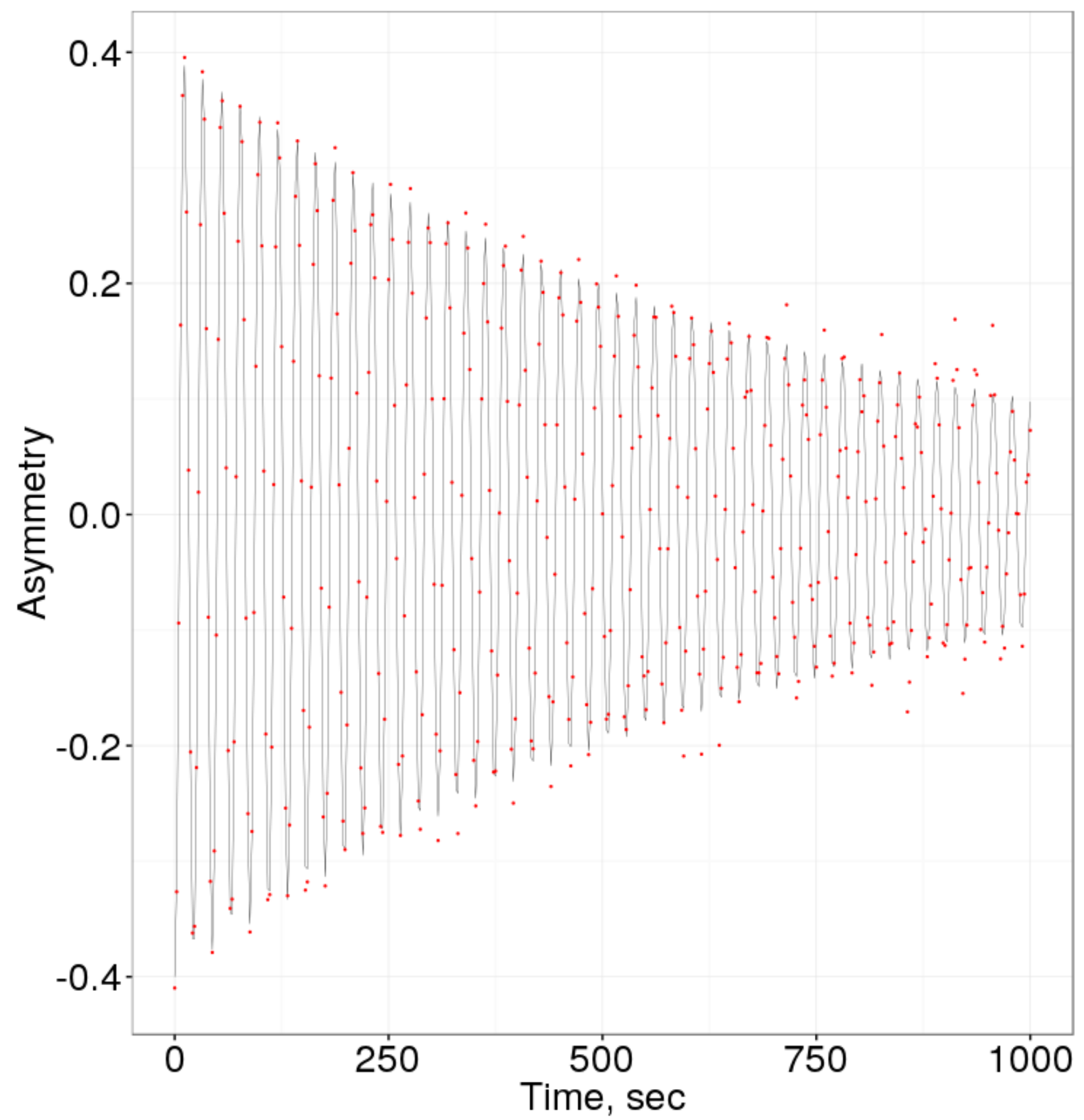

Figure A.4: Expectation value (red line) and sample measurements (black dots) of the cross-section asymmetry. 


\section{Bibliography}

[1] Trodden M. Electroweak baryogenesis. Rev Mod Phys. 1999 10;71:1463-1500. Available from: https://link.aps.org/doi/10. 1103/RevModPhys .71.1463.

[2] Christenson JH, Cronin JW, Fitch VL, Turlay R. Evidence for the $2 \pi$ Decay of the $K_{2}^{0}$ Meson. Phys Rev Lett. 1964 7;13:138-140. Available from: https://link.aps.org/doi/10.1103/PhysRevLett.13.138.

[3] Harris PG. The Neutron EDM Experiment. arXiv:07093100 [hepex];Available from: http://arxiv.org/abs/0709.3100.

[4] JEDI Collaboration;. Available from: http://collaborations. fz-juelich.de/ikp/jedi/about/introduction.shtml.

[5] Smith JH, Purcell EM, Ramsey NF. Experimental Limit to the Electric Dipole Moment of the Neutron. Phys Rev. 1957 10;108:120-122. Available from: https://link.aps.org/doi/10. 1103/PhysRev. 108.120.

[6] Baker CA, Doyle DD, Geltenbort P, Green K, van der Grinten MGD, Harris PG, et al. Improved Experimental Limit on the Electric Dipole Moment of the Neutron. Phys Rev Lett. 2006 9;97:131801. Available from: https://link.aps.org/doi/10.1103/PhysRevLett.97. 131801.

[7] Baker CA, Doyle DD, Geltenbort P, Green K, van der Grinten MGD, Harris PG, et al. Baker et al. Reply:. Phys Rev Lett. 2007 
4;98:149102. Available from: https://link.aps.org/doi/10.1103/ PhysRevLett.98.149102.

[8] Brown HN, Bunce G, Carey RM, et al. Precise Measurement of the Positive Muon Anomalous Magnetic Moment. Phys Rev Lett. 2001 3;86:2227-2231. Available from: https://link.aps.org/doi/10. 1103/PhysRevLett.86.2227.

[9] Bennett GW, Bousquet B, Brown HN, et al. Improved limit on the muon electric dipole moment. Phys Rev D. 2009 9;80:052008. Available from: https://link.aps.org/doi/10.1103/PhysRevD. 80.052008 .

[10] Farley FJM, Jungmann K, Miller JP, et al. New Method of Measuring Electric Dipole Moments in Storage Rings. Phys Rev Lett. 2004 7;93:052001. Available from: https://link.aps.org/doi/10.1103/ PhysRevLett.93.052001.

[11] srEDM Collaboration;. Available from: https://www.bnl.gov/ edm/.

[12] Anastassopoulos D, Anastassopoulos V, Babusci D, et al. AGS Proposal: Search for a permanent electric dipole moment of the deuteron nucleus at the $10^{-29} e \cdot \mathrm{cm}$ level. BNL; 2008. Available from: https://www.bnl.gov/edm/files/pdf/deuteron_ proposal_080423_final.pdf.

[13] Abusaif F, et al. Feasibility Study for a Storage Ring to Search for Electric Dipole Moments of Charged Particles; 2019. Available from: https//doi.org/xxxxx.

[14] Morse WM, Orlov YF, Semertzidis YK. rf Wien filter in an electric dipole moment storage ring: The "partially frozen spin" effect. Phys Rev ST Accel Beams. 2013 11;16:114001. Available from: https: //link.aps.org/doi/10.1103/PhysRevSTAB.16.114001. 
[15] Saleev A, Nikolaev NN, Rathmann F, et al. Spin tune mapping as a novel tool to probe the spin dynamics in storage rings. Phys Rev Accel Beams. 2017 7;20:072801. Available from: https://link.aps. org/doi/10.1103/PhysRevAccelBeams .20.072801.

[16] Slim J, Gebel R, Heberling D, et al. Electromagnetic Simulation and Design of a novel Waveguide RF Wien Filter for Electric Dipole Moment Measurements of Protons and Deuterons. Nuclear Instruments and Methods in Physics Research Section A: Accelerators, Spectrometers, Detectors and Associated Equipment;828:116124. Available from: http://www.sciencedirect.com/science/ article/pii/S0168900216303710.

[17] Slim J, for the JEDI Collaboration. First commissioning results of the waveguide RF Wien filter. Hyperfine Interactions;240(1):7. Available from: https://doi.org/10.1007/s10751-018-1547-6.

[18] Valetov E. FIELD MODELING, SYMPLECTIC TRACKING, AND SPIN DECOHERENCE FOR EDM AND MUON G-2 LATTICES. Michigan State University. Michigan, USA;. Available from: http://collaborations.fz-juelich.de/ikp/jedi/ public_files/theses/valetovphd.pdf.

[19] Mane SR. Spin Wheel. arXiv:150901167 [physics]. 2015 9;ArXiv: 1509.01167. Available from: http://arxiv.org/abs/1509.01167.

[20] Senichev Y, Aksentev A, Ivanov A, Valetov E. Frequency domain method of the search for the deuteron electric dipole moment in a storage ring with imperfections. arXiv:171106512 [physics]. 2017 11;ArXiv: 1711.06512. Available from: http://arxiv.org/abs/ 1711.06512 .

[21] Anastassopoulos V, Andrianov S, Baartman R, et al. A storage ring experiment to detect a proton electric dipole moment. Review of Scientific Instruments. 2016;87(11):115116. Available from: https: //aip.scitation.org/doi/abs/10.1063/1.4967465. 
[22] Koop IA. Asymmetric energy colliding ion beams in the EDM storage ring. In: Beam Dynamics and Electromagnetic Fields. Shanghai, China; 2013. p. 1961-1963. Available from: http://accelconf .web. cern.ch/accelconf/ipac2013/papers/tupwo040.pdf.

[23] Aksentev AE, Senichev YV. Statistical precision in charged particle EDM search in storage rings. Journal of Physics: Conference Series. 2017 12;941. Available from: http://stacks.iop.org/1742-6596/ $941 / i=1 / a=012083$.

[24] Dennis Eversmann. Analysis of the Spin Coherence Time at the Cooler Synchrotron COSY [master's thesis]. RWTH Aachen University;. Available from: http://collaborations.fz-juelich.de/ ikp/jedi/public_files/theses/DEMasterarbeit.pdf.

[25] NLREG package documentation;. Available from: https://cran. r-project.org/web/packages/nlreg/index.html.

[26] Beal SL, Sheiner LB. Heteroscedastic Nonlinear Regression. Technometrics. 1988;30(3):327-338. Available from: https://www. tandfonline.com/doi/abs/10.1080/00401706.1988.10488406.

[27] Senichev Y, Zyuzin D. SPIN TUNE DECOHERENCE EFFECTS IN ELECTRO- AND MAGNETOSTATIC STRUCTURES. In: Beam Dynamics and Electromagnetic Fields. vol. 5. Shanghai, China: JACoW; 2013. p. 2579-2581. OCLC: 868251790. Available from: https://accelconf.web.cern.ch/accelconf/ IPAC2013/papers/wepea036.pdf.

[28] Senichev Y, Andrianov S, Ivanov A, Chekmenev S, 3tin Berz, Valetov E. INVESTIGATION OF LATTICE FOR DEUTERON EDM RING. In: Modeling of current and future machnies. Shanghai, China; 2015. Available from: http://accelconf.web.cern.ch/AccelConf/ ICAP2015/papers/modbc4.pdf. 
[29] Senichev Y, Andrianov S, Berz M, Chekmenev S, Ivanov A, Lehrach A, et al. Quasi-frozen Spin Method for EDM Deuteron Search. In: Beam dynamics and EM fields; 2015. .

[30] Shatunov YM. Polarized beams in accelerators and storage rings. Novosibirks, Russia: SB RAS press; 2015.

[31] Berz M, Makino K. COSY Infinity website. 220 Trowbridge Rd, East Lansing, MI 48824, USA;. Available from: cosyinfinity.org.

[32] Berz M, Makino K. COSY INFINITY 10.0 Beam Physics Manual. 220 Trowbridge Rd, East Lansing, MI 48824, USA; 2017.

[33] Berz M, Makino K. COSY INFINITY 10.0 Programmer's manual. 220 Trowbridge Rd, East Lansing, MI 48824, USA; 2017.

[34] Bräutigam W, Brings R, Gebel R, Maier R, Schnase A, Jungwirth $\mathrm{HN}$. $\mathrm{H}^{-}$-Operation of the Cyclotron 7IC as Injector for the Cooler Synchrotron COSY-Jülich. In: 15th Int. Cyclotron Conf. and Their Applications (Cyclotrons'98). Caen, France: JACoW; 1998. p. 654657.

[35] Bechstedt U, et al. Status of the Cooler Synchrotron COSY Juelich. Conf Proc. 2001;C0106181:3350-3352. [,3350(2001)].

[36] Lehrach A, Lorentz B, Morse W, Nikolaev N, Rathmann F. Precursor Experiments to Search for Permanent Electric Dipole Moments (EDMs) of Protons and Deuterons at COSY. arXiv:12015773 [hepex, physics:nucl-ex, physics:physics];Available from: http://arxiv. org/abs/1201.5773.

[37] Lehrach A. Beam and Spin Dynamics for Storage Ring Based EDM Search. In: Proceedings, 6th International Particle Accelerator Conference (IPAC 2015): Richmond, Virginia, USA, May 3-8, 2015; 2015. p. WEAB2. Available from: http://accelconf.web.cern. ch/AccelConf/IPAC2015/papers/weab2.pdf. 
[38] Eversmann D, Hejny V, Hinder F, et al. New Method for a Continuous Determination of the Spin Tune in Storage Rings and Implications for Precision Experiments. Phys Rev Lett. 2015 8;115:094801. Available from: https://link.aps.org/doi/10. 1103/PhysRevLett.115.094801.

[39] Guidoboni G, Stephenson E, Andrianov S, et al. How to Reach a Thousand-Second in-Plane Polarization Lifetime with 0.97-GeV/c Deuterons in a Storage Ring. Phys Rev Lett. 2016 7;117:054801. Available from: https://link.aps.org/doi/10. 1103/PhysRevLett.117.054801.

[40] Wagner $\mathrm{T}$, on behalf of JEDI Collaboration. Beam-based alignment tests at the Cooler Synchrotron (COSY). Hyperfine Interact;239(1):61. Available from: https://doi.org/10.1007/ s10751-018-1539-6.

[41] Bagdasarian Z, Bertelli S, Chiladze D, et al. Measuring the polarization of a rapidly precessing deuteron beam. Phys Rev ST Accel Beams. 2014 5;17:052803. Available from: https://link.aps.org/ doi/10.1103/PhysRevSTAB.17.052803.

[42] Greta Guidoboni. Overview of Spin Coherence Time study results at COSY; Topical meeting of Spin Tracking for Precision Measurements (IPAC15). Available from: http://collaborations.fz-juelich. de/ikp/jedi/public_files/workshop/G.Guidoboni_IPAC15.pdf.

[43] Guidoboni G, on behalf of JEDI Collaboration. Spin coherence time studies of a horizontally polarized deuteron beam at COSY. Physica Scripta. 2015 11;T166:014036. 